\title{
AD-HOC REGIONAL COVERAGE CONSTELLATIONS OF CUBESATS USING SECONDARY LAUNCHES
}

\author{
A Thesis \\ Presented to \\ the Faculty of California Polytechnic State University, \\ San Luis Obispo
}

\author{
In Partial Fulfillment \\ of the Requirements for the Degree \\ Master of Science in Aerospace Engineering
}

by

Guy G. Zohar

March 2013 
(C) 2013

Guy G. Zohar

ALL RIGHTS RESERVED 


\section{COMMITTEE MEMBERSHIP}

TITLE:

AUTHOR:

DATE SUBMITTED:

COMMITTEE CHAIR:

COMMITTEE MEMBER:

COMMITTEE MEMBER:

COMMITTEE MEMBER:
Ad-Hoc Regional Coverage Constellations of CubeSats using Secondary Launches

Guy G. Zohar

March 2013

Dr. Jordi Puig-Suari, Professor

Cal Poly Aerospace Engineering Department

Dr. Kira J Abercromby, Assistant Professor

Cal Poly Aerospace Engineering Department

Daniel J Wait; Lecturer

Cal Poly Aerospace Engineering Department

Dr. Gerald L. Shaw; Senior Research Engineer

SRI International 


\begin{abstract}
Ad-Hoc Regional Coverage Constellations of CubeSats Using Secondary Launches Guy G. Zohar
\end{abstract}

As development of CubeSat based architectures increase, methods of deploying constellations of CubeSats are required to increase functionality of future systems. Given their low cost and quickly increasing launch opportunities, large numbers of CubeSats can easily be developed and deployed in orbit. However, as secondary payloads, CubeSats are severely limited in their options for deployment into appropriate constellation geometries.

This thesis examines the current methods for deploying cubes and proposes new and efficient geometries using secondary launch opportunities. Due to the current deployment hardware architecture, only the use of different launch opportunities, deployment direction, and deployment timing for individual cubes in a single launch are explored. The deployed constellations are examined for equal separation of Cubes in a single plane and effectiveness of ground coverage of two regions. The regions examined are a large near-equatorial zone and a medium sized high latitude, high population density zone.

Results indicate that simple deployment strategies can be utilized to provide significant CubeSat dispersion to create efficient constellation geometries. The same deployment strategies can be used to develop a multitude of differently dispersed constellations. Different launch opportunities can be utilized to tailor a constellation for a specific region or mission objective. Constellations can also be augmented using multiple launch opportunities to optimize a constellation towards a specific mission or region. The tools developed to obtain these results can also be used to perform specific analysis on any region in order to optimize future constellations for other applications. 


\section{ACKNOWLEDGEMENTS}

There is a significant amount of people that I want to thank for their continuous help in my long path to completing this work. First, I would like to thank my advisor Dr. Jordi Puig-Suari for helping me define and shape what was an initially vague idea regarding constellations into my completed thesis. I also want to thank Justin Foley and Roland Coelho at CubeSat for their perpetual help and patience. As I progressed through my work and continuously needed new, and at times, odd information they quickly and promptly worked with me to find a solution. I want to thank Dr. Kira Abercromby for assisting and advising me whenever possible while my primary advisor was on sabbatical. Her help in the early formation of my thesis allowed me to develop the concepts required for this research.

Thank you to Analytics Graphic Inc. (www.agi.com) for the free use of their Systems Tool Kit (STK) software versions 9 and 10. Access to the professional license and MATLAB connect modules was critical in development of this work.

I want to thank John Erickson and the whole Range Safety team at Vandenberg AFB for their continuous support of my research. With my ever-changing schedule their patience and support was invaluable. Lastly to my friends, family, and fantastic girlfriend; thank you for all your help and support. In various ways, all of them kept my drive to complete this work going. 


\section{TABLE OF CONTENTS}

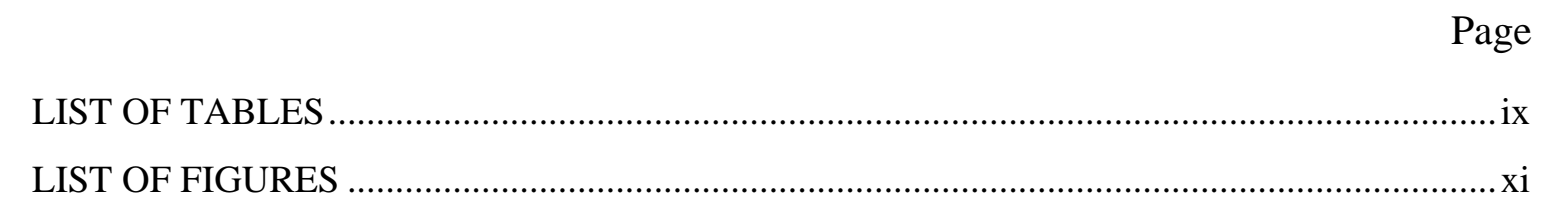

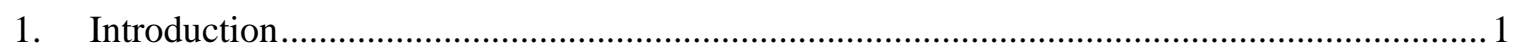

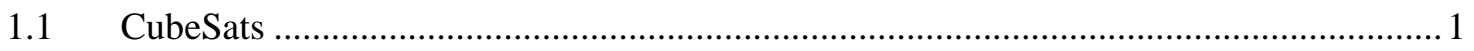

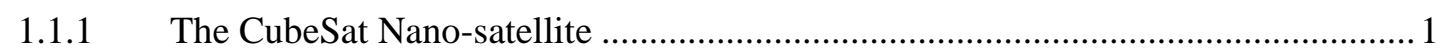

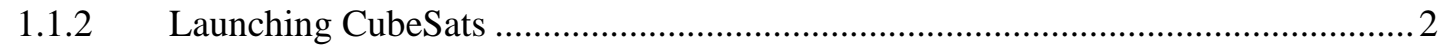

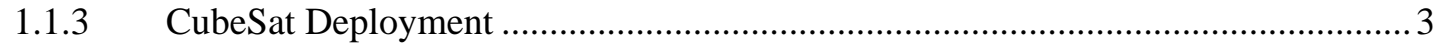

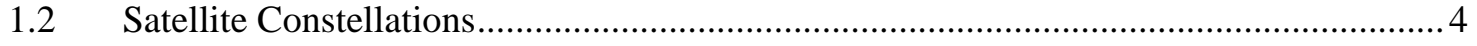

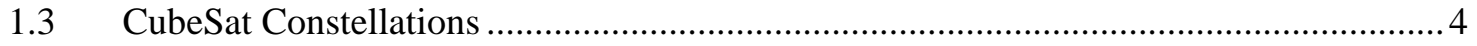

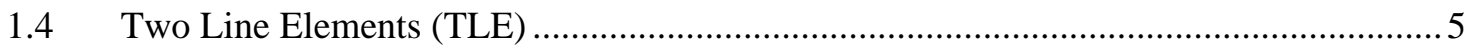

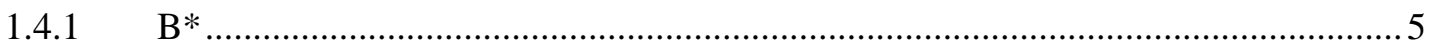

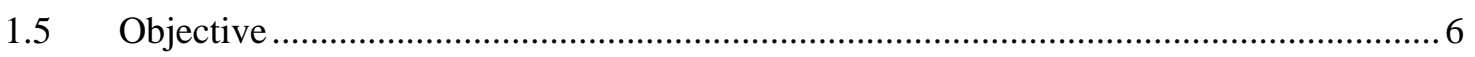

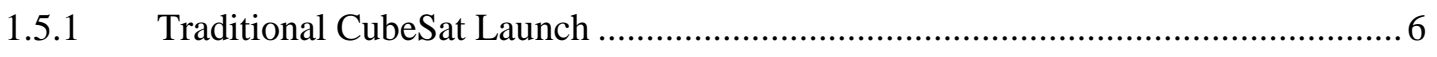

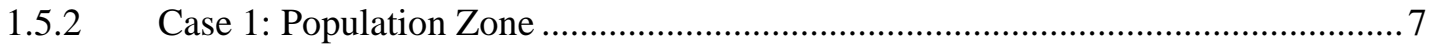

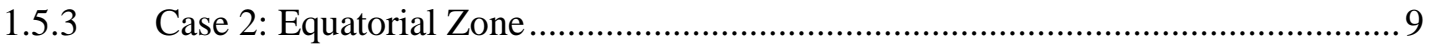

1.5.4 Constellation Development and Breakdown.......................................................... 10

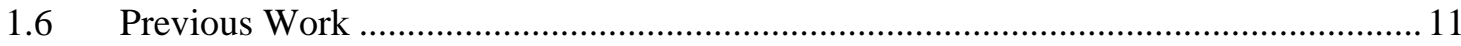

1.6.1 CubeSat Constellation Analysis for Data Relaying ............................................. 11

1.6.2 Controlling CubeSat Constellations using Drag Differential................................. 12

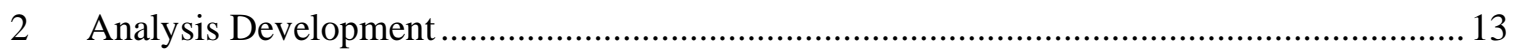

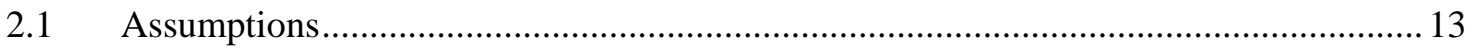

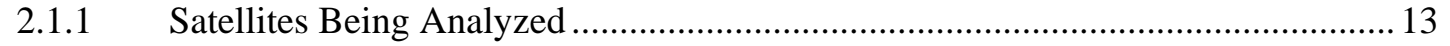

2.1.2 Drag Effects On Relative CubeSat Position............................................................ 14

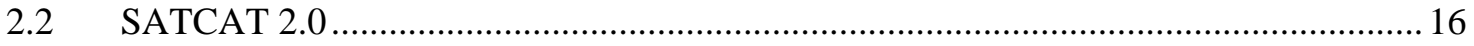

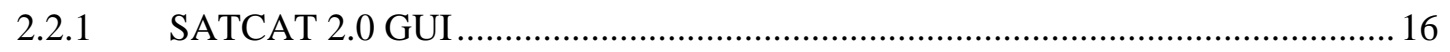

2.2.2 Using SATCAT 2.0 for CubeSat deployment...................................................... 19

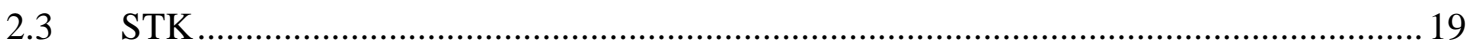

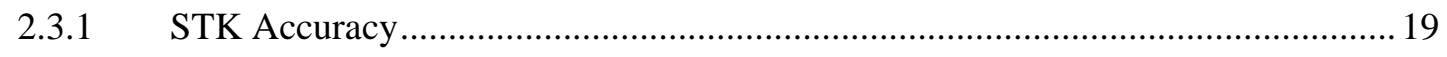

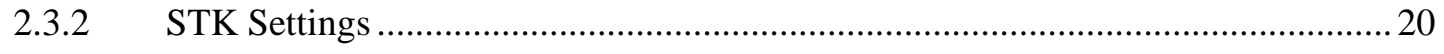

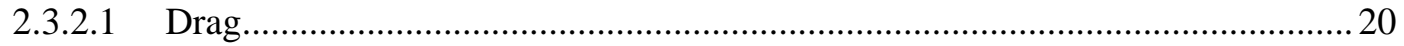

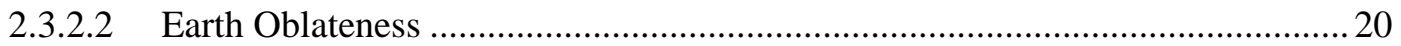




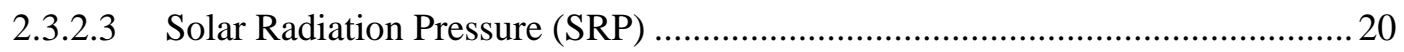

2.4 Relative Motion Equations (CW - Hill approximations) …....................................... 20

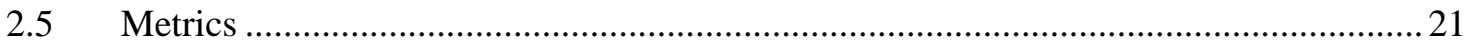

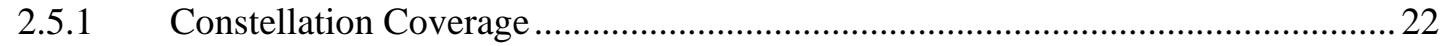

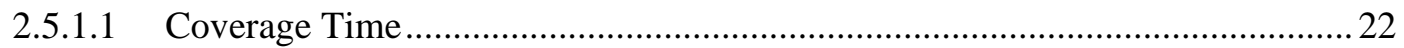

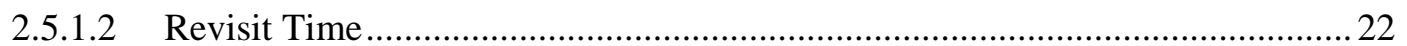

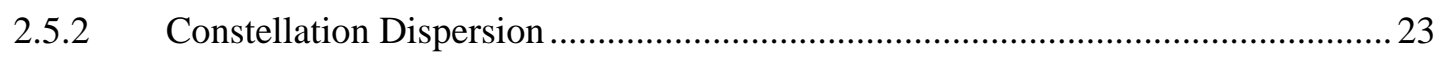

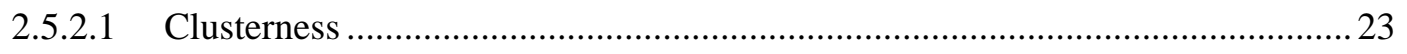

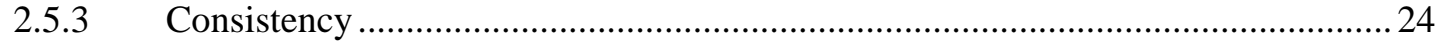

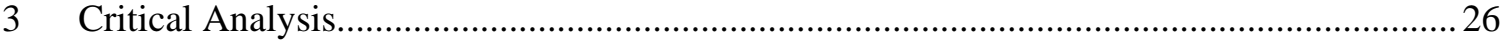

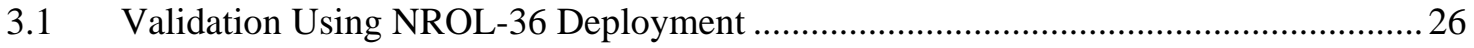

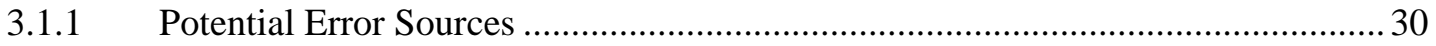

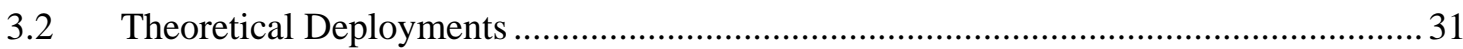

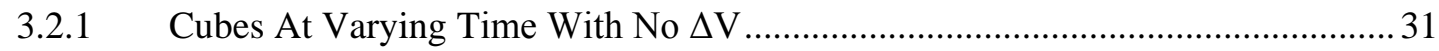

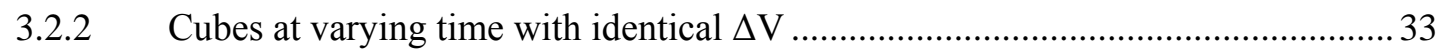

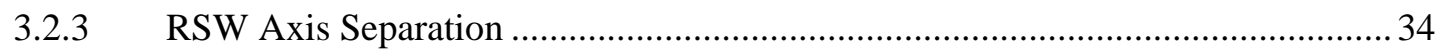

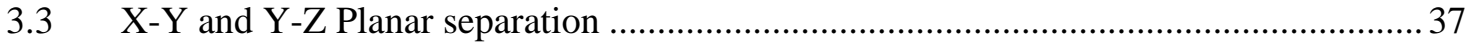

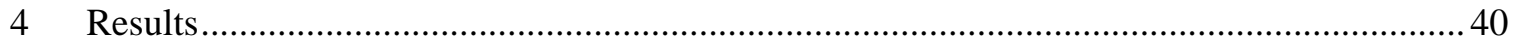

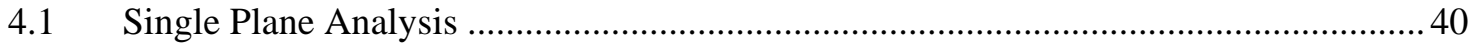

4.1.1 Angle based Semi-Circle Separation (X-Y plane) ............................................... 40

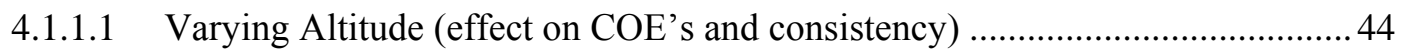

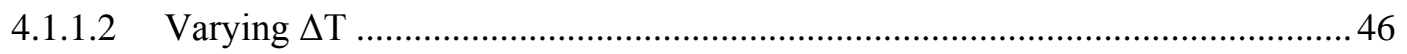

4.1.2 Equally Distributed Y-Vector Component Based Semi-Circle Separation ............ 48

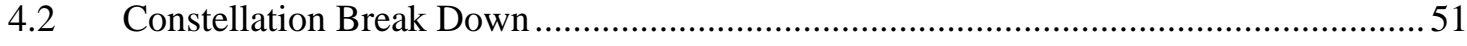

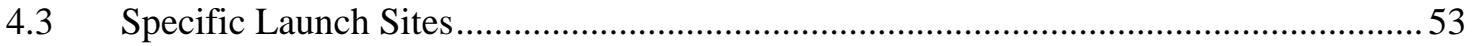

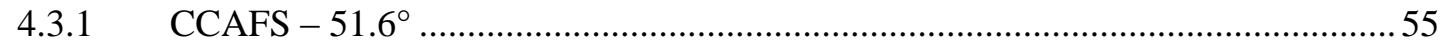

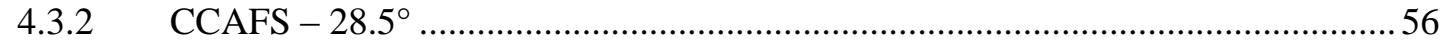

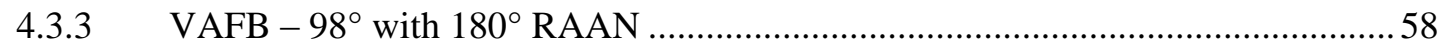

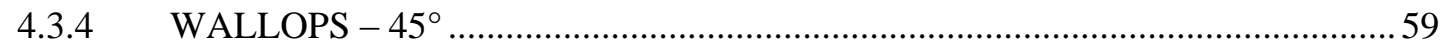

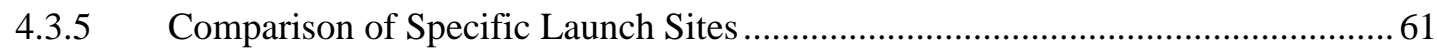

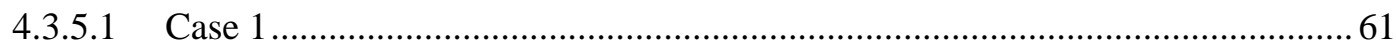

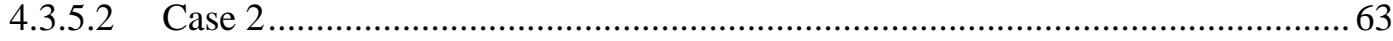

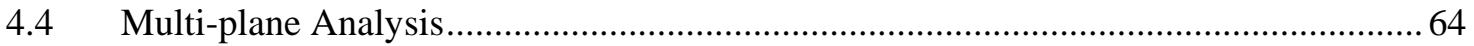




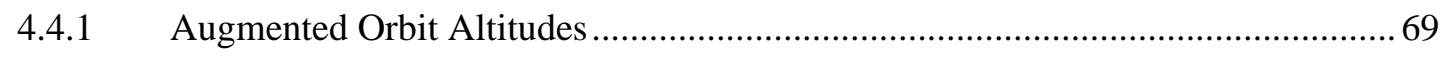

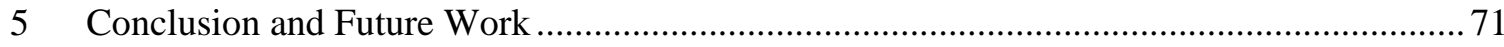

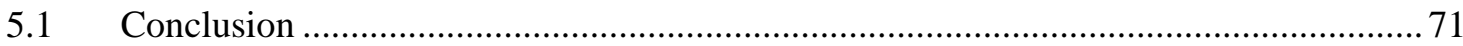

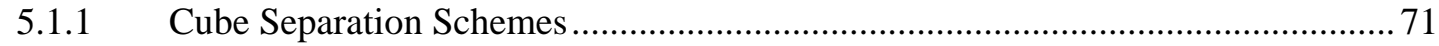

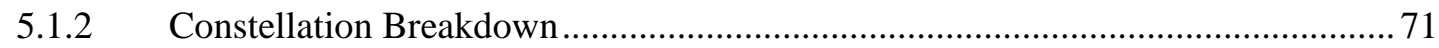

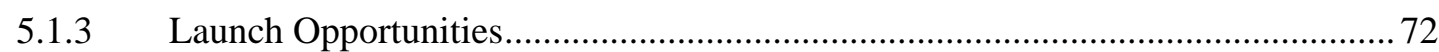

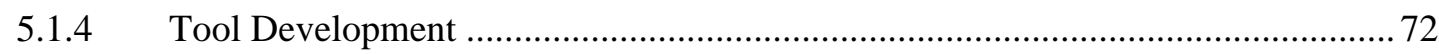

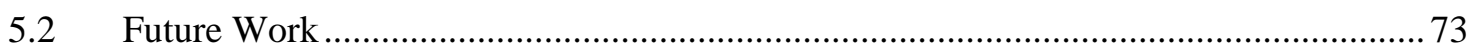

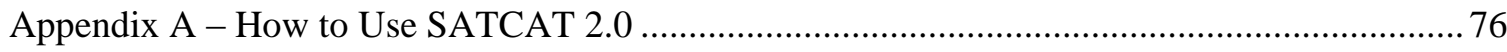

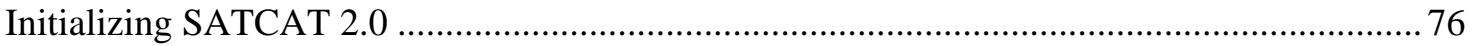

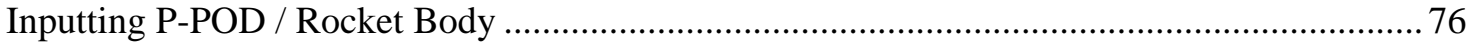

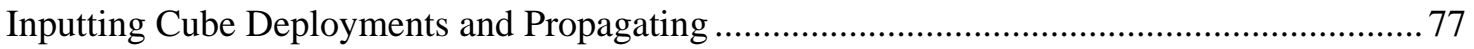

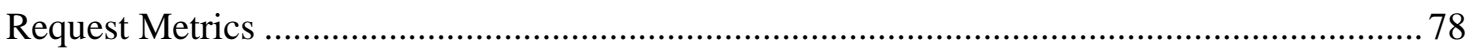

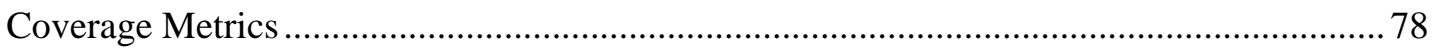

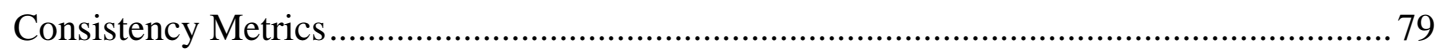

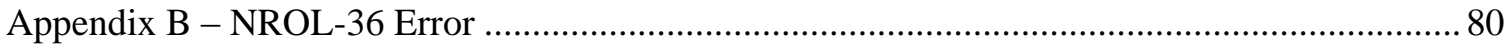




\section{LIST OF TABLES}

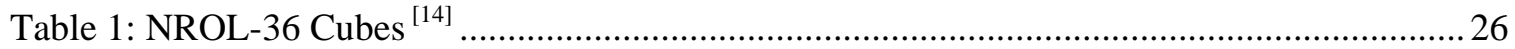

Table 2: 3U Cube - PPOD separation speed for given mass........................................................29

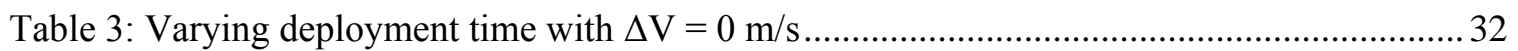

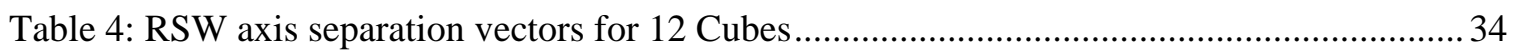

Table 5: X-Y and Y-Z separation direction based on degree offset from +Y............................. 37

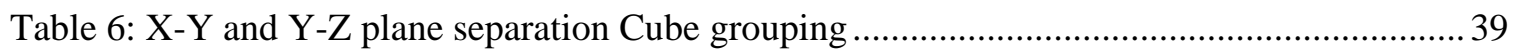

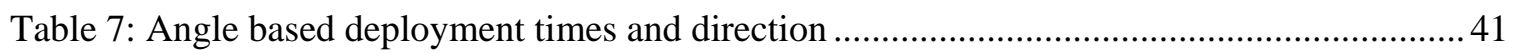

Table 8: Varying altitudes using angle-based semi-circle deployment .................................... 44

Table 9: Deployment time and Clusterness due to varying altitude .......................................... 45

Table 10: Constellation development due to increasing separation time;

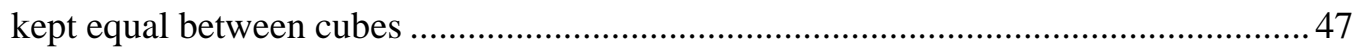

Table 11: Varying relative separation time between each Cube separation event......................... 47

Table 12: Y-vector based semi-circle deployment times and direction ..................................... 48

Table 13: Angle based separation orbital period at initial full deployment ..................................52

Table 14: $\Delta \mathrm{V}$ for each cube in angle based semi-circle separation ...........................................53

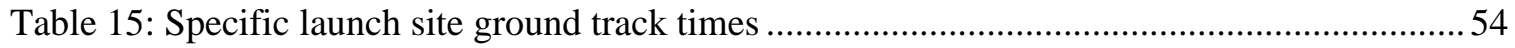

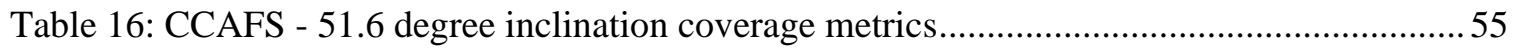

Table 17: CCAFS - 28.5 inclination coverage metrics ............................................................5 57

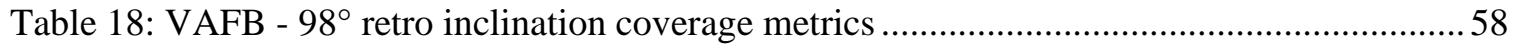

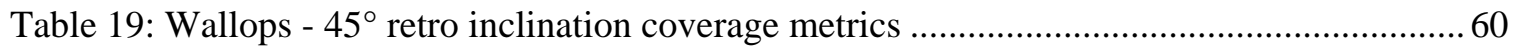

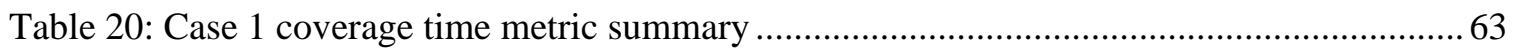

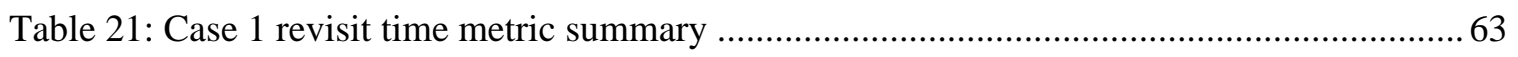

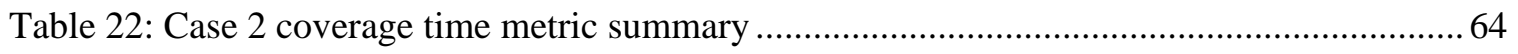

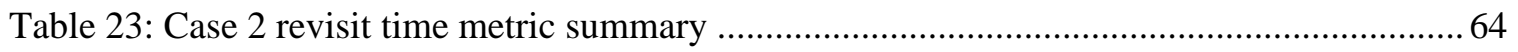


Table 24: Two plane constellation coverage metrics...

Table 25: Augmented two plane constellation metrics 


\section{LIST OF FIGURES}

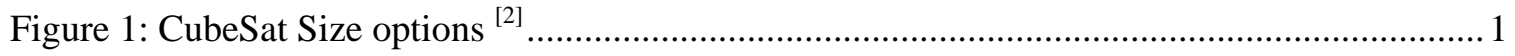

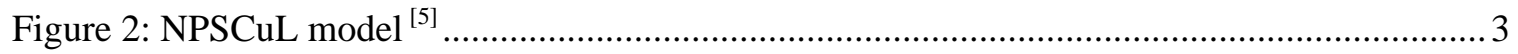

Figure 3: Case 1 area definition over Western Europe …........................................................ 8

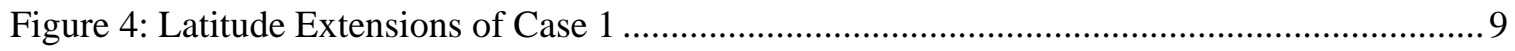

Figure 5: Case 2 area definition over Sub-Saharan Africa ....................................................... 10

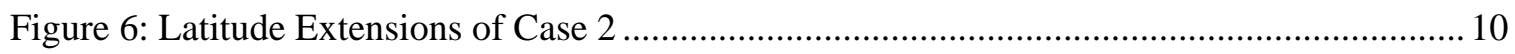

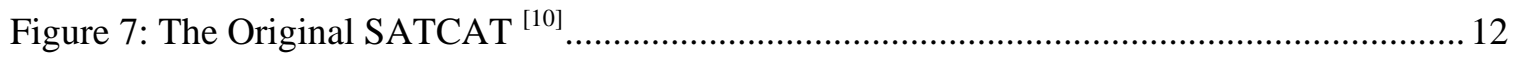

Figure 8: B* term for RAX-2 over approximately 225 days ................................................. 14

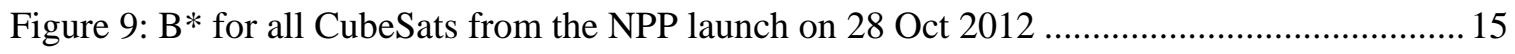

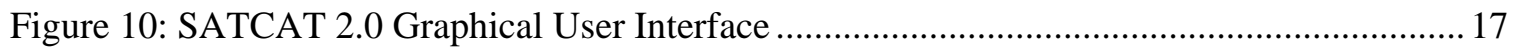

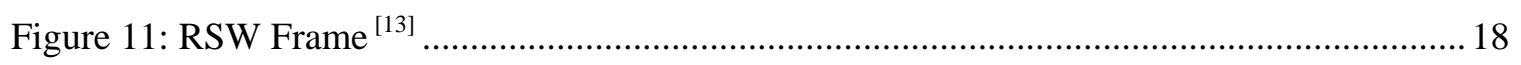

Figure 12: Error between STK analysis and TLEs for RAX-2 …........................................ 19

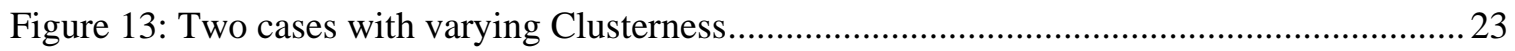

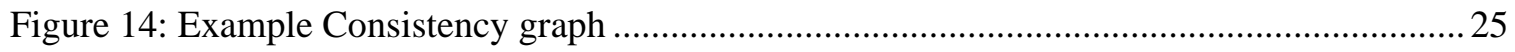

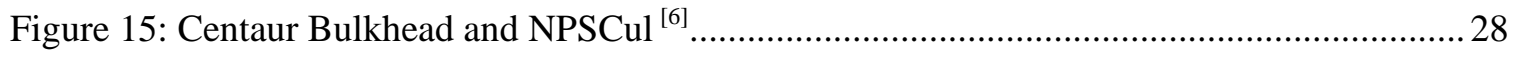

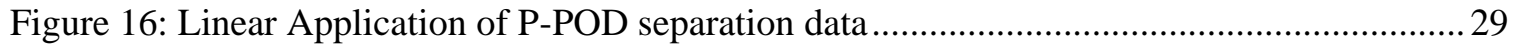

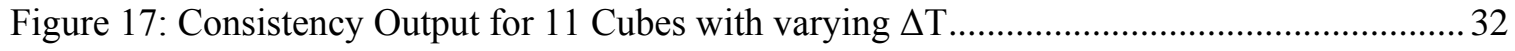

Figure 18: Consistency Output for 11 Cubes with varying $\Delta \mathrm{T}$ and $\Delta \mathrm{V}=1.5 \mathrm{~m} / \mathrm{s} \ldots \ldots \ldots \ldots \ldots \ldots \ldots . . . . . . . . .33$

Figure 19: 12 Cubes separated on RSW axis with no time between separations.......................... 35

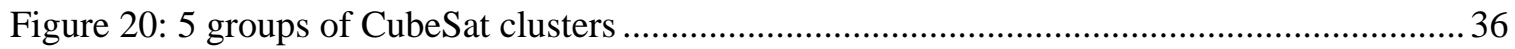

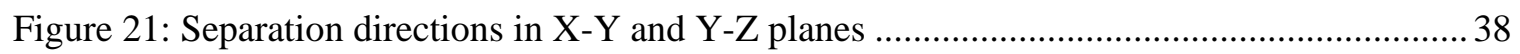

Figure 22: Consistency for separation in X-Y and Y-Z planes.................................................. 38

Figure 23: Superposition of 8 Cubes separated in semi-circle scheme ...................................... 41

Figure 24: Angle based semi-circle separation consistency .................................................... 42 
Figure 25: Simulated cube positions after 45 days from semi-circle spacing

Figure 26: Varying perigee altitude vs. time to full deployment ........................................... 45

Figure 27: Equal Y-vector semi-circle separation consistency ............................................... 49

Figure 28: Simulated cube positions after 45 days from equally distributed Y-vector separation 50

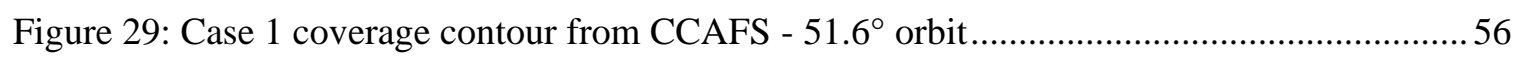

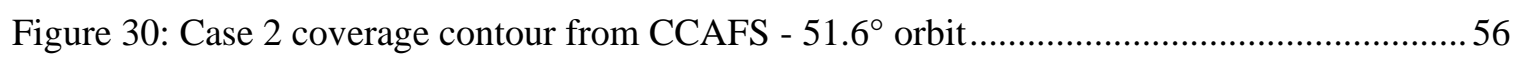

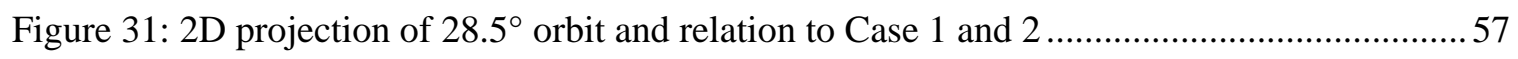

Figure 32: Case 2 coverage contour from CCAFS - $28.5^{\circ}$ orbit ............................................... 58

Figure 33: Case 1 coverage contours from VAFB $-98^{\circ}$ retro orbit............................................59

Figure 34 Case 1 coverage contours from VAFB - $98^{\circ}$ retro orbit .............................................59

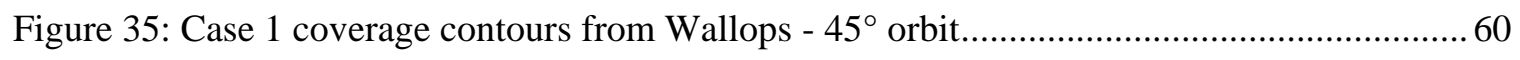

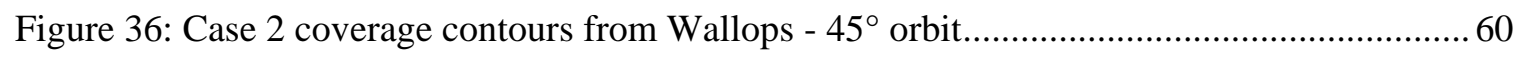

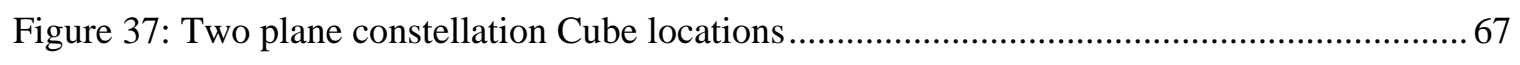

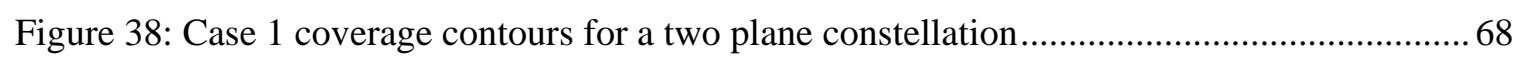

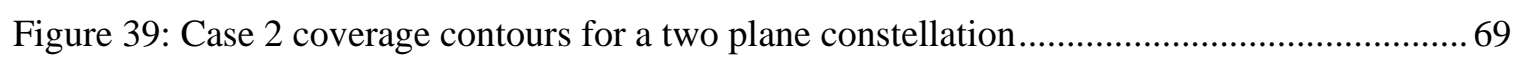




\section{Introduction}

\subsection{CubeSats}

\subsubsection{The CubeSat Nano-satellite}

CubeSats are defined as $10 \mathrm{~cm}$ cubes with a mass less than $1.33 \mathrm{~kg}^{[1]}$ and are a subset of Nanosatellites. This dimension is referred to as the $1 \mathrm{U}$ (or 1 unit) size, as CubeSats can come in larger sizes. Larger form CubeSat, measured in how many "U's" they are, generally come in a 3U size. $3 \mathrm{U}$ CubeSats retain the $10 \mathrm{~cm} \times 10 \mathrm{~cm}$ base but have an increased height of $30 \mathrm{~cm}$ and are nominally required to be less that $4 \mathrm{~kg}$ in mass. Both a $1 \mathrm{U}$ and a $3 \mathrm{U}$ CubeSat are shown in Figure 1 below.
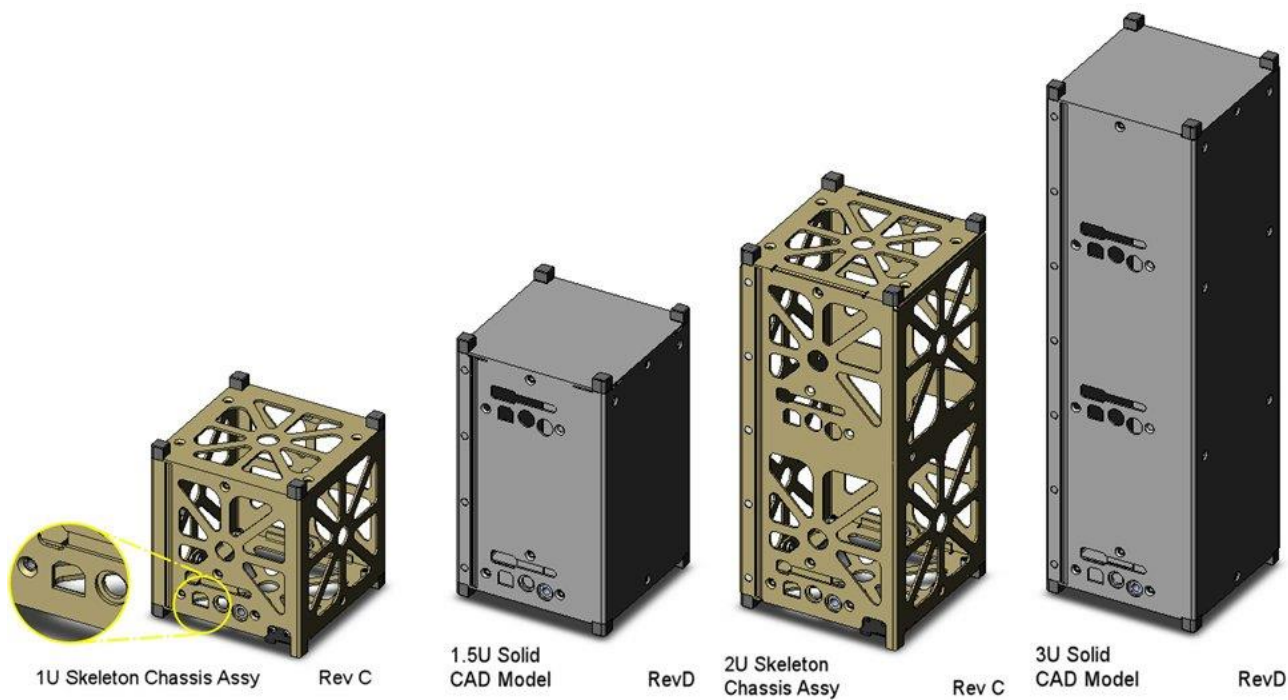

Figure 1: CubeSat Size options ${ }^{[2]}$

CubeSats are traditionally launched as a "ride along" payload allowing for extremely low cost launches. Due to the low cost launches, universities, high schools, and private firms are able to develop, build, and launch a nano-satellite into orbit. ${ }^{[3]}$ Given their low launch costs and quick development cycles CubeSats have been gaining popularity in instrument testing and novel architecture designs. 


\subsubsection{Launching CubeSats}

In order to launch a CubeSat it must first be placed into a deploying mechanism. A standard launch interface a standard mechanism called the Poly Pico-satellite Orbital Deployer (P-POD) was developed $^{[4]}$ to allow for a standard interface onto a rocket body. The P-POD fits up to a total of 3U's worth of Cubes, which can be comprised of a 3U CubeSat, three 1U CubeSats, or any other option adding to a total of $3 \mathrm{U}$. The P-POD is then attached to an upper stage rocket body as a secondary payload and deploys the cubes once the rocket has reached orbit. By not being a primary payload the CubeSat developers are able to launch their satellite at extremely low prices, which would have otherwise prohibited them from launching the satellite.

Final approval for a CubeSat to be attached as a secondary payload resides with the owner of the primary payload due to the potential increased risk the CubeSat could pose to the primary payload. The P-POD provides and safe method to integrate CubeSats to the launch vehicle which increases the likelihood of approval by the primary payload. Launch providers are more likely to allow a CubeSats on board as the P-POD ensures that CubeSat deployables are encased within by the P-POD and that the CubeSat remains powered off until after deployment.

Due to the high demand of launch opportunities the Naval Postgraduate School CubeSat Launcher (NPSCuL), shown in Figure 2 below, was developed to integrate multiple P-PODS into one system. The current NPSCuL can be integrated into multiple launch vehicles and holds eight P-PODS. [5] 


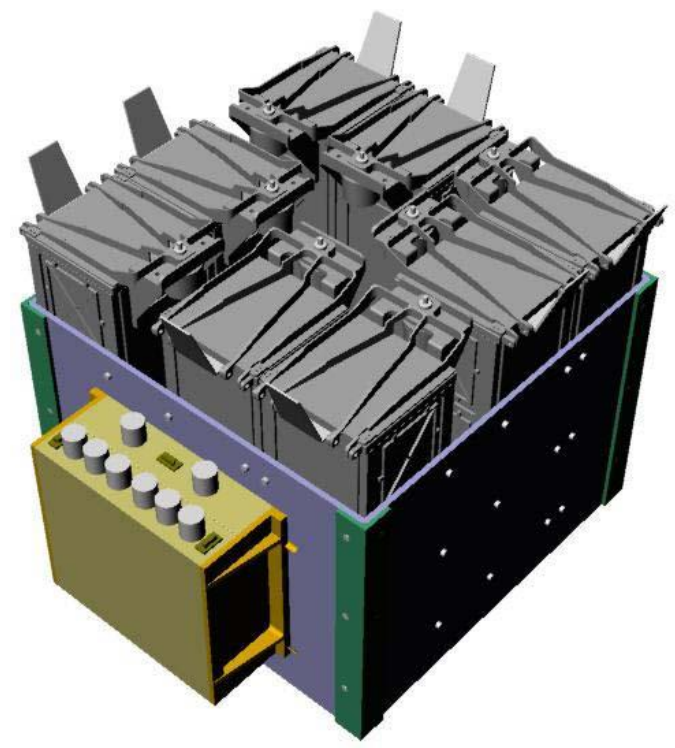

Figure 2: NPSCuL model ${ }^{[5]}$

The NPSCuL was first used on the Atlas V launch vehicle on the newly created Aft Bulkhead Carrier plate for the NROL-36 launch. It is expected that each Atlas V launch will have an NPSCuL system on-board drastically increasing the launch capabilities of CubeSats ${ }^{[6]}$.

\subsubsection{CubeSat Deployment}

Once the main P-POD door opens a compressed spring forces the CubeSats out of the P-Pod. All of the Cubes insides are forced out at a speed determined by their mass and the spring parameters. The spring design is constant for each P-POD causing cubes of varying mass to leave the P-POD at different speeds, usually between 1.2 and $1.7 \mathrm{~m} / \mathrm{s}$, in accordance with Newton's Second Law of Motion. The launch vehicle's upper rocket body attitude determines the direction in which the CubeSat is deployed. The Cubes are also separated at a specific, recurring time span, such as a deployment every 3 minutes. Currently, neither direction nor timing is designed for. The CubeSats are deployed in whatever direction the launch vehicle's upper stage happens to be aligned with a constant time between each release. 


\subsection{Satellite Constellations}

Satellite Constellations are used for missions that require more than one satellite and allow for dramatically more complex missions. Potential options include global and regional coverage for voice, data, communications relays, and multi-point science measurement. A prime example of a satellite constellation is the Global Positioning System (GPS) which uses 24 satellites to provide position and time data to a ground user ${ }^{[7]}$. The Iridium satellite constellation relays data for satellite phones and provides telephone connectivity worldwide using 66 low earth orbit satellites

${ }^{[8]}$. While constellations can be extremely useful they are generally extremely costly. For example, the GPS constellation is funded at over 1.3 billion dollars a year ${ }^{[7]}$. Further, the upcoming private Iridium NEXT constellation is estimated to cost approximately $\$ 2.9$ billion ${ }^{[8]}$.

\subsection{CubeSat Constellations}

A constellation formed with CubeSats has many distinct advantages when compared to a single CubeSats or even full scale satellite constellations. A CubeSat constellation is able to utilize the same aspects of a larger constellation but at a fraction of the price. By utilizing the small form factor of CubeSats and extremely low cost launches multiple satellites can be placed into a constellation at a fraction of the cost of a traditional constellation.

Just like the CubeSat allowed smaller developers to launch and operate satellites, a CubeSat constellation allows smaller developers to launch and operate their own constellations. This could provide further costs savings if a developer chooses to launch their own CubeSat constellation instead of paying to use Iridium, ORBCOMM, or other commercial constellation. Developers are be able to tailor a constellation for a more specific use, such as providing additional coverage for a specific region or decreasing the times between overpasses to a location.

While there are significant positives in utilizing a CubeSat constellation there are also significant issues with implementing them. The biggest problem with a CubeSat constellation is also the 
most important trait of a CubeSat, being a secondary payload. Constellation development can be extremely difficult because CubeSats cannot select their own orbit. Current and many planned CubeSat constellations do not require specific orbit characteristics but instead utilize CubeSats for multi-node science measurements.

In order to properly populate a constellation the cubes must be deployed using a specific scheme. However; as explained in Section 1.1.3 the CubeSat deployment direction is currently erratic and varies with the rocket upper body while the deployment is set to a repeating regular interval. This method of deployment leads to unknown constellations that cannot be tailored properly. In order to develop a tailored CubeSat constellation new deployment methods must be explored.

\subsection{Two Line Elements (TLE)}

A critical data set for CubeSats is the satellite ephemeris data provided by the Joint Space Operations Command (JSpOC), formerly NORAD, based at Vandenberg AFB. This data is called a "Two Line Element" (TLE) due to its format and includes information on the satellites: epoch, mean motion, $\mathrm{B}^{*}$, inclination, right ascension of ascending node, mean anomaly, and mean motion. TLEs are determined with a variety of sensors available to JSpOC and are generally considered accurate after approximately two weeks due to repeated measurements of a satellite. They are especially useful for CubeSat developers who only have access to narrow beam-width antennas and must point them directly at a Cube in order to establish a communications link.

\subsubsection{B*}

Other than the orbit descriptor data, the TLE contains information collected by JSpOC on B* (BSTAR), a drag-like parameter. This metric provides information regarding the effects of drag on a satellite and is defined as ${ }^{[9]}$

$$
B^{*}=C_{d}\left(\frac{A}{m}\right)\left(\frac{\rho_{o}}{2}\right)
$$


where $\mathrm{C}_{\mathrm{d}}$ is the drag coefficient of the satellite, $A / m$ is the Area to mass ratio, and $\rho_{o}$ is an average atmospheric density of the earth. JSpOC sets $\rho_{o}$ at a constant value and allows $\mathrm{C}_{\mathrm{d}}$ to fluctuate even though in reality it is the opposite. This is done to allow JSpOC not to measure the actual atmospheric density and instead only measure the effect of the atmospheric density on the satellite.

$\mathrm{B}^{*}$ is often unused because the value is drastically skewed if a satellite has onboard propulsion methods. With CubeSats that do not have propulsion B* can provide insightful data as to how the Cube is being affected by drag. This is extremely useful when comparing the effects of drag against a constellation of CubeSats in order to understand the relative drag effects.

\subsection{Objective}

This thesis outlines the necessary requirements to deploy a constellation of traditionally launched CubeSats capable of covering a specified region. Different launch sites are examined and compared as Cubes have limited control over which launches they can ride along with. Multiple launches are also a feasible option when developing a large constellation. For this reason a multiple launch scenario is evaluated.

Two case regions are examined to test the robustness of deploying CubeSat constellations. The first case examines a medium sized circular area with a high population density. The second case examines a large equatorial area with large ranges in latitude and longitude.

\subsubsection{Traditional CubeSat Launch}

In order to ensure that developers directly apply the resulting analysis, the Constellation deployment must be constrained by the "traditional" method of launching Cubes. The key aspects of a CubeSat launch is that separation speed is controlled by the existing P-POD spring and that Cubes do not get to select their own orbits. ${ }^{[4]}$ 
Constraining the Cube separation to using the current P-POD spring hardware leaves the separation timing and direction as available variables in developing a constellation. Any modifications to the P-POD spring will result in less confidence in the overall P-POD system, a key requirement in obtaining secondary launch opportunities; leading to less available launches. Constraining the Cubes as a secondary payload requires analysis to be done on the orbits most commonly available. Because each launch of a primary vehicle may have a totally different orbit, the orbits selected are based on major launch facilities and common orbital inclinations launched from those facilities. The altitude of the orbits that the Cubes are analyzed in varies from 400 to $650 \mathrm{~km}$ based on the orbital lifetime and current CubeSat orbits. Each major launch site orbit is analyzed; after which Cubes are added to the constellation from two launch sites and re-analyzed.

\subsubsection{Case 1: Population Zone}

Due to potential interest by CubeSat constellation developers, a medium sized high population region is analyzed. The tested region must be far enough north or south, to avoid the equatorial region covered in Case 2, but not so far north or south as to become a polar coverage analysis. An area in the northern hemisphere is more beneficial to analyze given that the northern hemisphere is much more highly populated.

The region of Western Europe is be analyzed as that it meets the above requirement and includes many developers may find the region of particular interest for a CubeSat constellation. The population zone is modeled as a circle centered in France and extending radially into parts of Germany, Spain, the United Kingdom and other Western European nations as shown in Figure 3 below. 


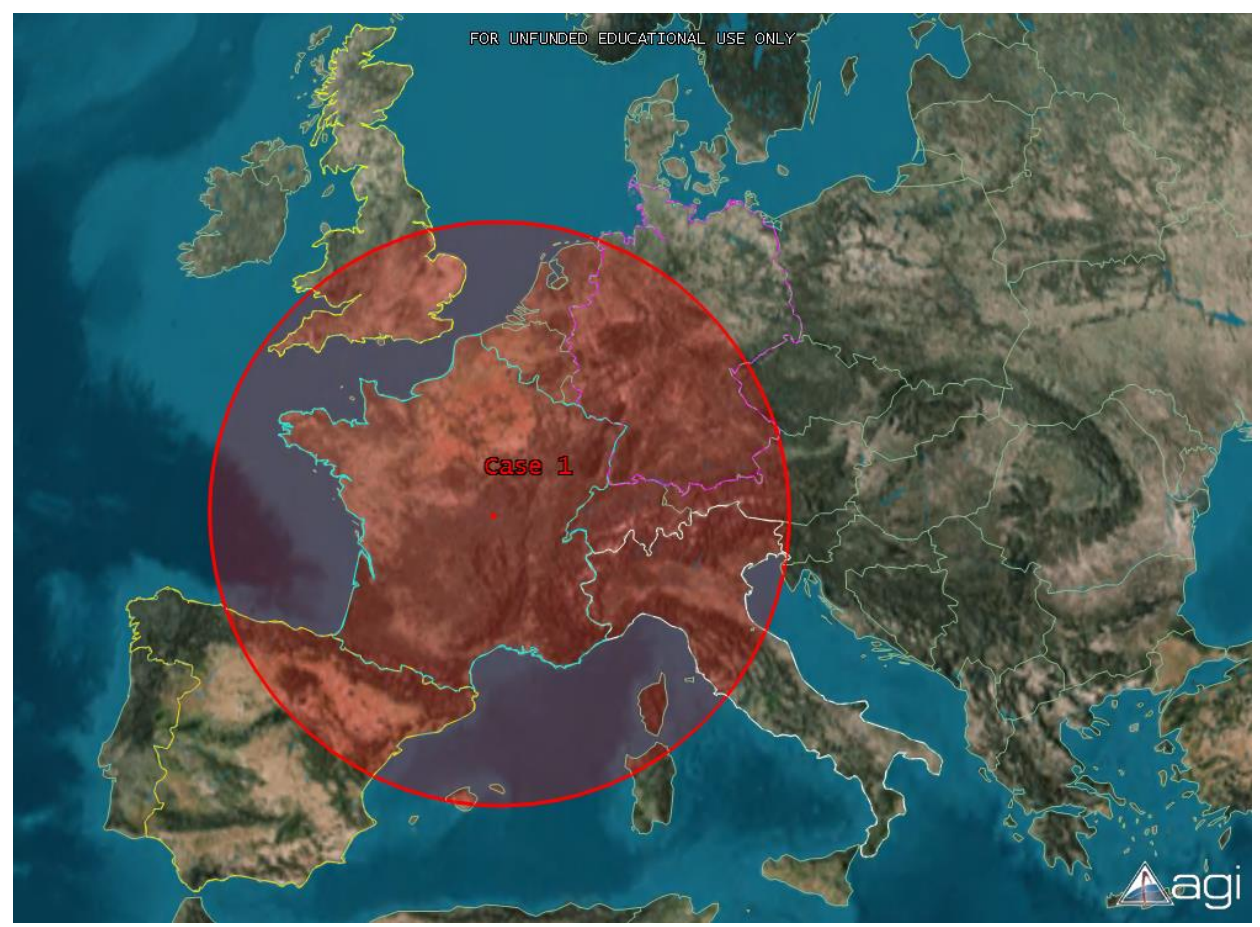

Figure 3: Case 1 area definition over Western Europe

The circle is centered at $\left\langle 46^{\circ} 42^{\prime} 0^{\prime \prime} \mathrm{N}, 3^{\circ} 30^{\prime} 0^{\prime \prime} \mathrm{E}>\right.$ with a radius of $800 \mathrm{~km}$ (497 miles), extending the northern and southern bounds of the region to approximately $54^{\circ} \mathrm{N}$ and $39.4^{\circ} \mathrm{N}$ respectively. The defined region has a surface area of $2.00609 \times 10^{6} \mathrm{~km}^{2}$.

Due to the physical nature of orbits the RAAN of the constellation will shift over time allowing the same constellation to be used over areas with the same latitude range but a different longitude range. This effect allows the this case to be expanded into constellation development for important regions such as Eastern Europe, East Asia, and the Northern portion of the United States as shown in Figure 4 below. 


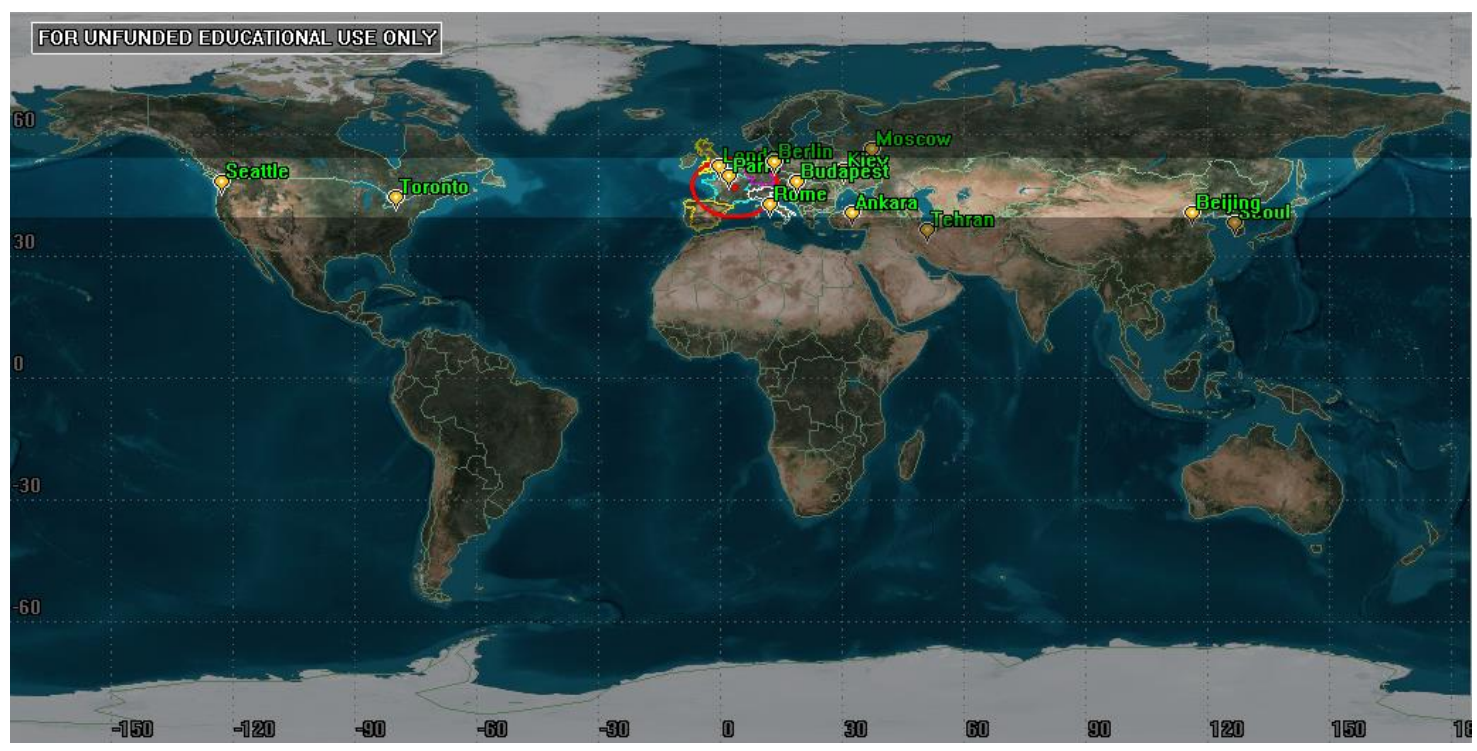

Figure 4: Latitude Extensions of Case 1

\subsubsection{Case 2: Equatorial Zone}

To ensure that the deployment methods developed are applicable to a large area of the earth, a large equatorial zone is required. In conjunction with the higher latitude area of Case 1, an equatorial area will validate the robustness of the deployment methods.

For Case 2, the sub-Saharan portion of the African continent is selected. The region transverses both sides of the equator and covers a wide range of latitude and longitude. As shown in Figure 5 below, the equatorial zone is modeled a square. It extends from $10^{\circ} \mathrm{N}$ to $35^{\circ} \mathrm{S}$ latitude and $0^{\circ}$ to $52^{\circ}$ degrees longitude; covering $2.72854 \times 10^{7} \mathrm{~km}^{2}$. 


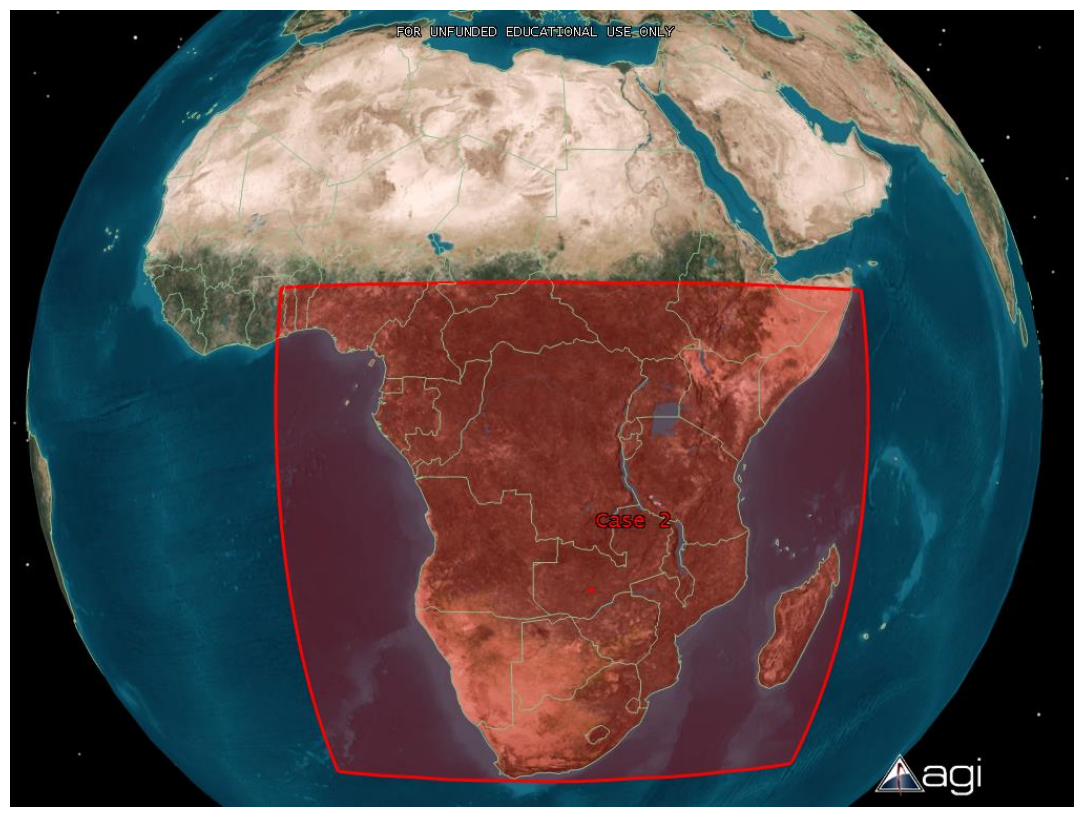

Figure 5: Case 2 area definition over Sub-Saharan Africa

As Case 1, the constellation will drift in RAAN over time allowing the deployment of Case 2 to apply to any region with latitudes between $10^{\circ}$ to $-35^{\circ}$; shown in Figure 6 below

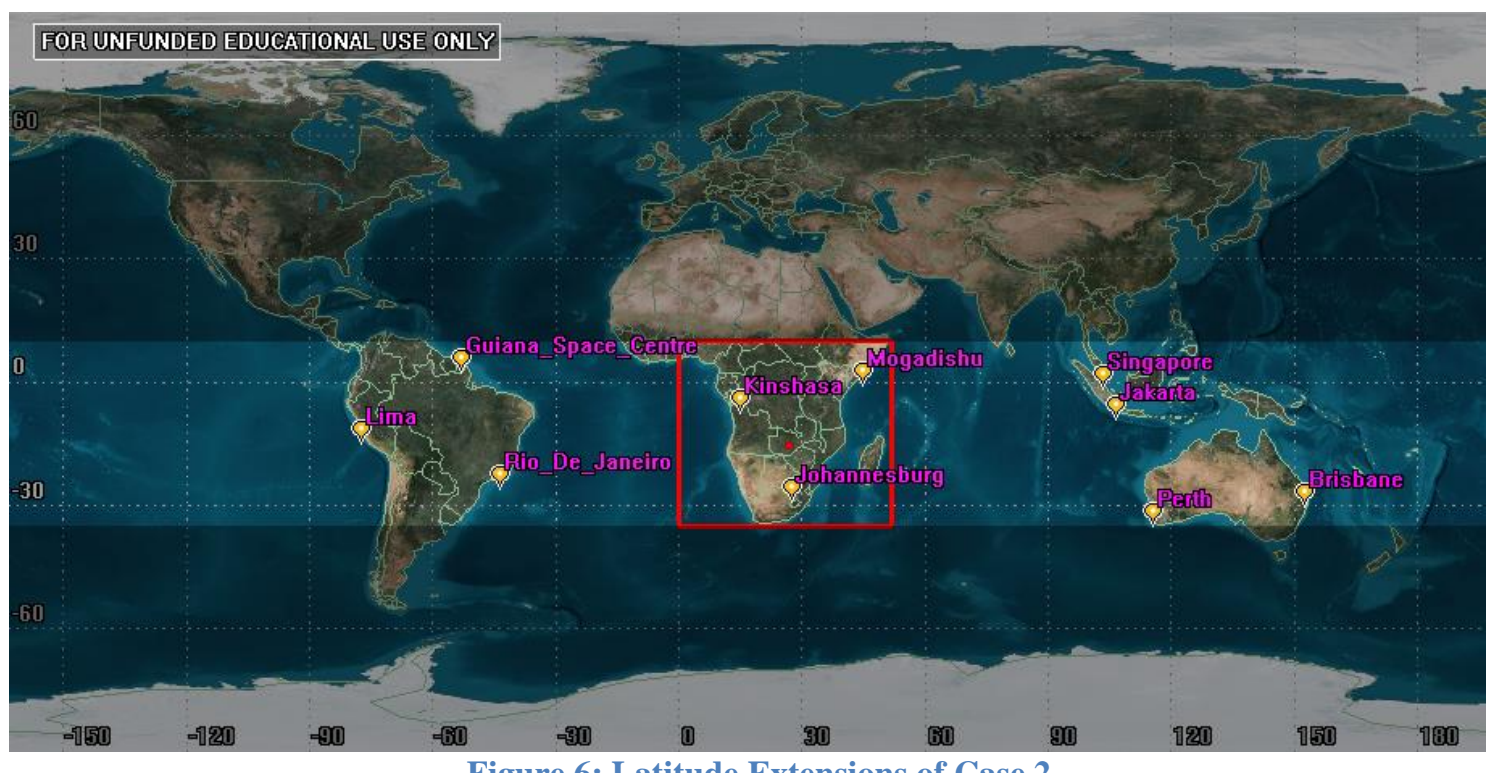

Figure 6: Latitude Extensions of Case 2

\subsubsection{Constellation Development and Breakdown}

For the purposes of the analysis in this paper a constellation will be considered developed when it has reached its best separation state. The best separation state will be defined in following 
sections based on various metrics. Due to the cyclical nature of the orbits once a constellation has reached this state it will immediately begin to breakdown. This breakdown will not be analyzed but the velocity change required to stop the constellation from breaking down will be provided.

\subsection{Previous Work}

There is a large collection of previous work regarding constellations and CubeSats but not a significant amount of working regarding CubeSat constellations. However, two previous papers provide important insight that assists in the analysis of this paper. These papers provide the baseline for feasible CubeSat constellations and ongoing research that allows for methods of maintain CubeSat constellations.

\subsubsection{CubeSat Constellation Analysis for Data Relaying}

CubeSat Constellation Analysis for Data Relaying ${ }^{[10]}$, by Brad Smalarz's, developed the Satellite Constellation Analysis Tool (SATCAT) utilizing the MATLAB integration module and Systems Tool Kit (STK) by AGI. SATCAT is a modular tool built as a MATLAB GUI which allows users to control STK using MATLAB's unique scripting features. SATCAT is built to allow new users to add or remove modules in order to change its functionality. SATCAT v1 (used in Mr.

Smalaraz's thesis) was designed to analyze the specific constellations being researched; however, SATCAT can be changed to perform any analysis available to STK. Figure 7 below shows the user interface of SATCAT. 


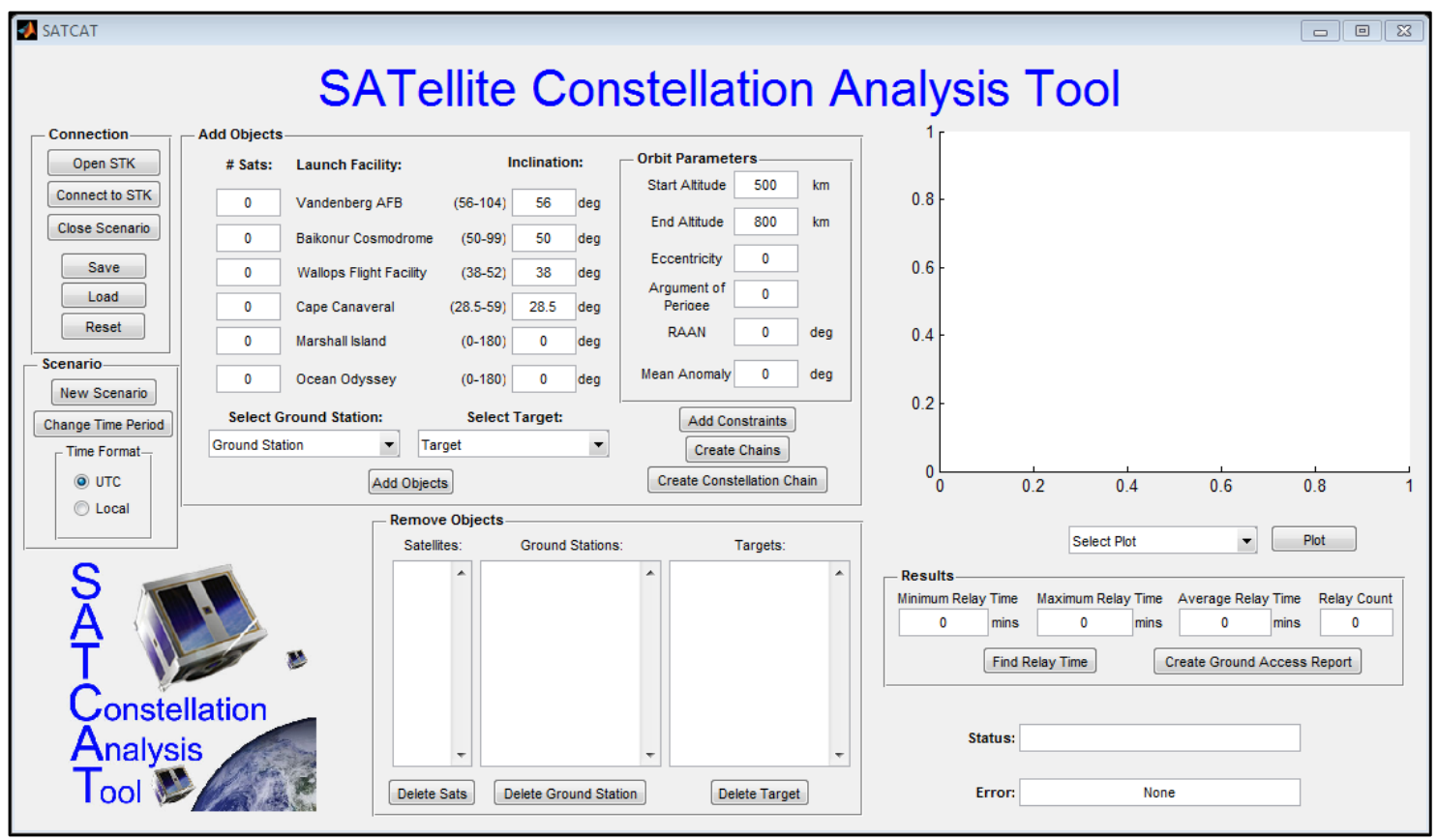

Figure 7: The Original SATCAT ${ }^{[10]}$

Using SATCAT Mr. Smalarz was able to analyze the performance of CubeSat-based store and forward communication constellations. This work showed that a CubeSat constellation was viable and useful. However, the scope of the thesis allowed the analysis to determine constellations without regard to the launch or deployment. Thus, Mr. Smalarz came to a conclusion that further work would be required in order to actually deploy the constellations necessary.

\subsubsection{Controlling CubeSat Constellations using Drag Differential}

Mr. Winetraub and Dr. Tamir at Israeli Aerospace Industries developed a method of controlling CubeSat constellations using differential drag in "Using Differential Drag for Management of Nano-Satellite Constellations". ${ }^{[1]}$ The developed method shows that it's possible to control and maintain and CubeSat constellation using a changing drag profile. This functionality can be critical as most cubes do not have on-board propulsion systems that are generally required in constellation station keeping. More importantly this functionality can be used to stop a constellation from drifting apart after being deployed into position. 


\section{Analysis Development}

Specific tools and baseline decisions are required In order to accurately analyze the deployment and development of CubeSat constellations. This allows for accurate and repeatable analysis of a multitude of Cube deployment scenarios.

\subsection{Assumptions}

\subsubsection{Satellites Being Analyzed}

A critical assumption that must be made for accurate analysis uses identical cubes with full attitude control. Due to its popularity a $4 \mathrm{~kg} 3 \mathrm{U}$ form factor Cube is the ideal choice for a CubeSat constellation developer and is the chosen satellite configuration in this analysis. As most constellations require attitude control, the analyzed cubes are fixed with a $30 \times 10 \mathrm{~cm}$ cross section in the RAM direction and a $10 \times 10 \mathrm{~cm}$ cross section in the NADIR. Each cube is also assumed to have a 30 degree cone half angle (60 degree total cone) sensor pointed NADIR for use in STK's coverage analysis.

The above cubes will experience identical perturbations that are functions of attitude, mass, and cross sectional area. The cubes will still experience different perturbations due to effects that are functions of satellite position. For example the effect of drag follows ${ }^{[9]}$ :

$$
\vec{a}_{\text {drag }}=-\frac{1}{2} C_{D} \frac{A}{m} \rho v_{r e l}^{2} \frac{\vec{v}_{r e l}}{\left|\vec{v}_{r e l}\right|}
$$

where $\mathrm{C}_{\mathrm{d}}$ is the drag coefficient of the satellite, $A / m$ is the Area to mass ratio, and $\mathrm{v}_{\text {rel }}$ is the satellite velocity to the moving atmosphere, assumed to be equal to the satellites orbital velocity. Unlike in Equation ( 1 ), $\rho$ in Equation ( 2 ) is the actual density of the Earth's atmosphere and not a reference density. This poses a significant problem for any predictive simulations due to the complexity in finding or predicting the density of the earth's atmosphere at the predicated 
location of the satellite. This is especially difficult given that this parameter is required potentially up to five years in advance in order to accurately simulate a satellites path.

Multiple methods are available for estimating the density of Earth's atmosphere and the effect of drag and include models such as: Jacchia, Exponential, GOST, and NRLMSISE 2000. Some of these models use analytic equations to solve for the earth's density; however, the more accurate models use measured space weather values from NOAA. STK has multiple built in models and downloads up-to-date space weather data for input into the appropriate model. Models that require space weather data must use predicted space weather values for simulations done for future timeframes.

\subsubsection{Drag Effects On Relative CubeSat Position}

As shown in section 1.4.1, B* can be used as a measure of how much a satellite is being affected by drag. In order to better understand the effects of drag on a CubeSat the B* term of a CubeSat's historical TLE's are examined. In Figure 8, the B* term of the RAX-2 CubeSat launched with on 28 Oct 2011 with Suomi NPP satellite ${ }^{[12]}$ are examined for approximately 225 days to understand the changing effect of drag on the Cube.

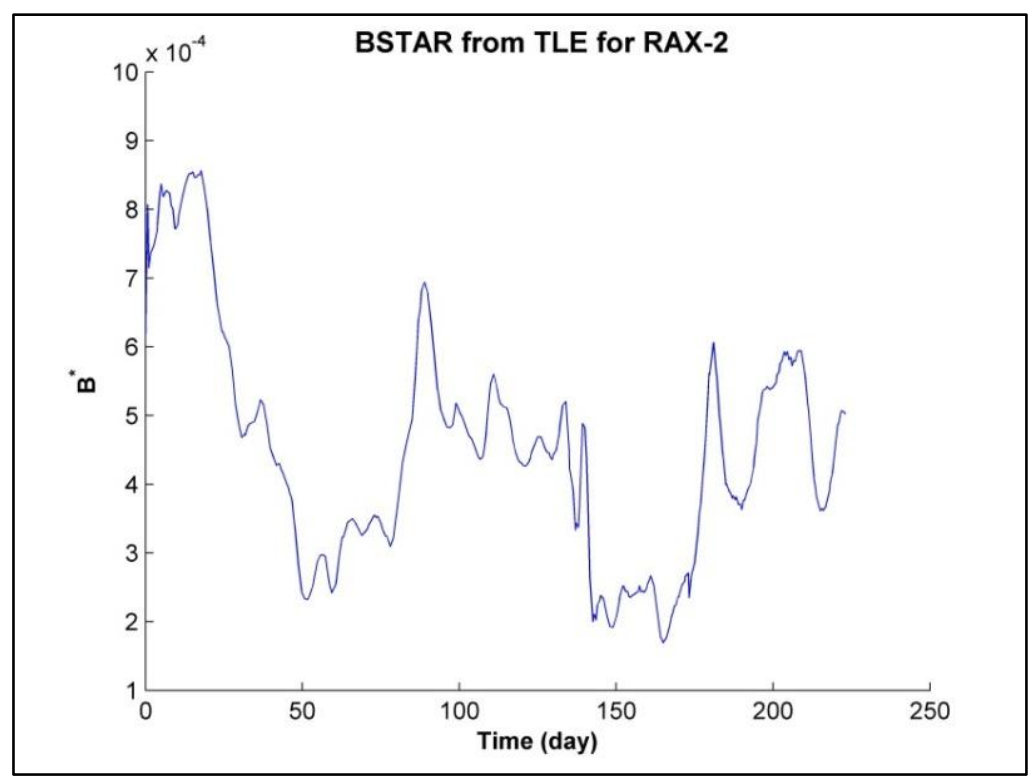

Figure 8: B* term for RAX-2 over approximately 225 days 
It can be seen that $\mathrm{B}^{*}$ changes by nearly four times over the time range examined. As the average area, mass, and drag coefficient of RAX-2 do not change over this time, the density of the Earth's atmosphere must have changed as expected thus yielding a varying drag force on the Cube. This unpredictable and dramatic change in drag perturbation must be mitigated in order to develop and maintain a CubeSat constellation.

All the Cubes from the NPP launch on 28 Oct 2011 were examined, as shown in Figure 9 below, to understand the effect of the changing drag on multiple CubeSats.

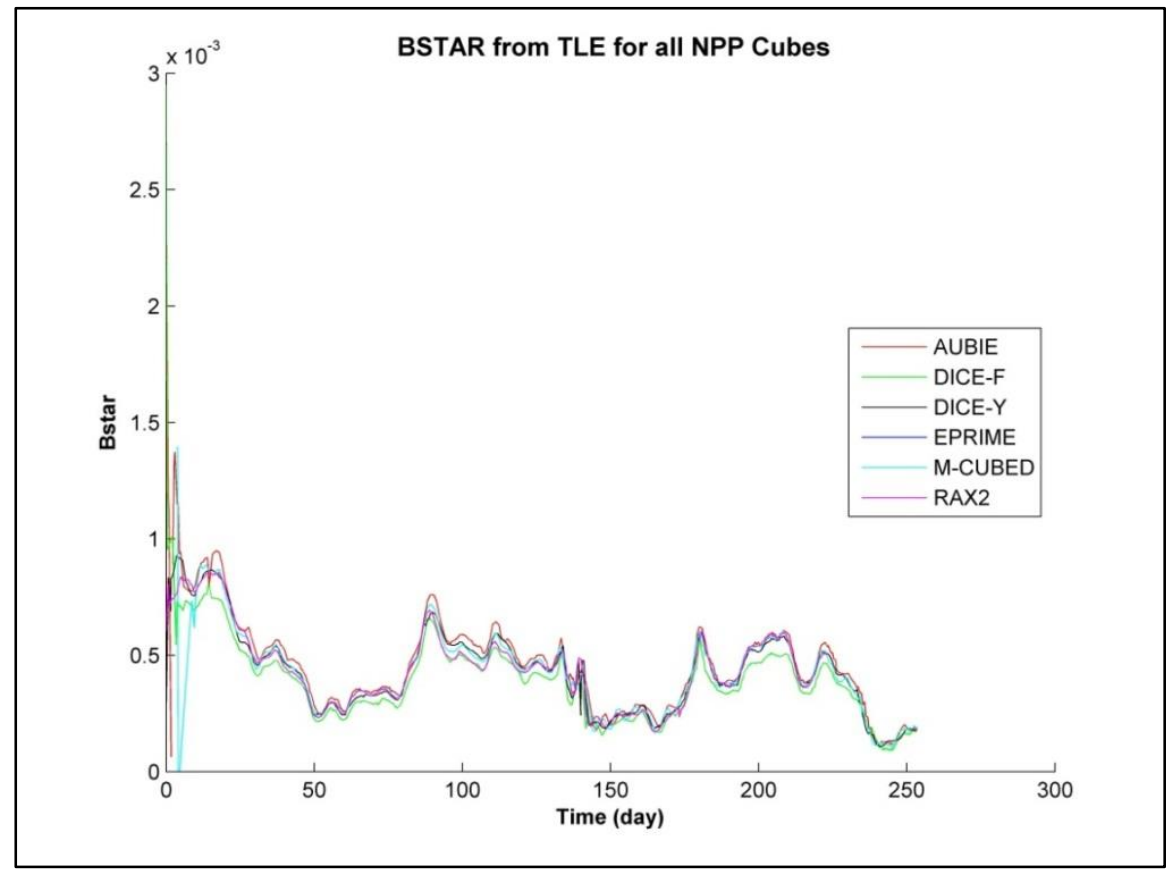

Figure 9: $\mathbf{B}^{*}$ for all CubeSats from the NPP launch on 28 Oct 2012

Initially the $\mathrm{B}^{*}$ terms for each Cube varies wildly in comparison to one another due to the errors associated with the TLE. Once the TLE converges and the error subsides it can be seen that all Cubes follow a distinct trend with the B* term nearly identical. The effect of drag on the cubes changes identically for each Cube. Since the Cubes in the NPP launch are not identical they will be affected by the force of drag differently in accordance with Equation ( 2 ). However, identical cubes, with identical attitude, in similar orbits will experience an identical force of drag. CubeSats in identical orbits won't be subjected to different atmospheric densities. 
Since the P-POD can only impart a velocity change of approximately $1.5 \mathrm{~m} / \mathrm{s}$, the P-POD cannot significantly change the orbit of a deployed CubeSat from that of the rocket body. Hence all Cubes from one launch vehicle, assuming the vehicle does not perform any orbit changing maneuvers, are in similar enough orbits for the cubes to be subjected to identical Earth atmospheric density. Cubes deployed from separate launch vehicles will experience a different drag force if they are deployed into a different altitude orbit and experience an identical drag force amongst themselves.

Designed constellations will have a different real world deployment due to the changing and unpredictable drag effect. Nevertheless, this will affect all Cubes in similar orbits equally resulting in a negligible relative location deviation between simulation and real world testing. CubeSats in other orbit altitudes will also experience the same negligible change in relative location and a change in the absolute location.

\subsection{SATCAT 2.0}

In order to effectively analyze the deployment schemes accurately and repeatedly a new version of SATCAT was developed. SATCAT is a MATLAB based Graphical User Interface (GUI) that uses the MATLAB - STK connect module. This module allows MATLAB to issue commands directly to STK.

SATCAT 1.0, shown in Figure 7, was used for "store and forward" constellation analysis but was built to be modular. SATCAT 2.0, shown in Figure 10, leverages the modules of SATCAT 1.0 that connect to STK and create a new scenario as a baseline and is designed to simulate and analyze CubeSat deployments and constellations.

\subsubsection{SATCAT 2.0 GUI}

SATCAT 2.0 keeps the Connection and Scenario modules from SATCAT 1.0 as well as a few minor GUI Commands. The rest of the modules in SATCAT 2.0 were created for version 2.0. 


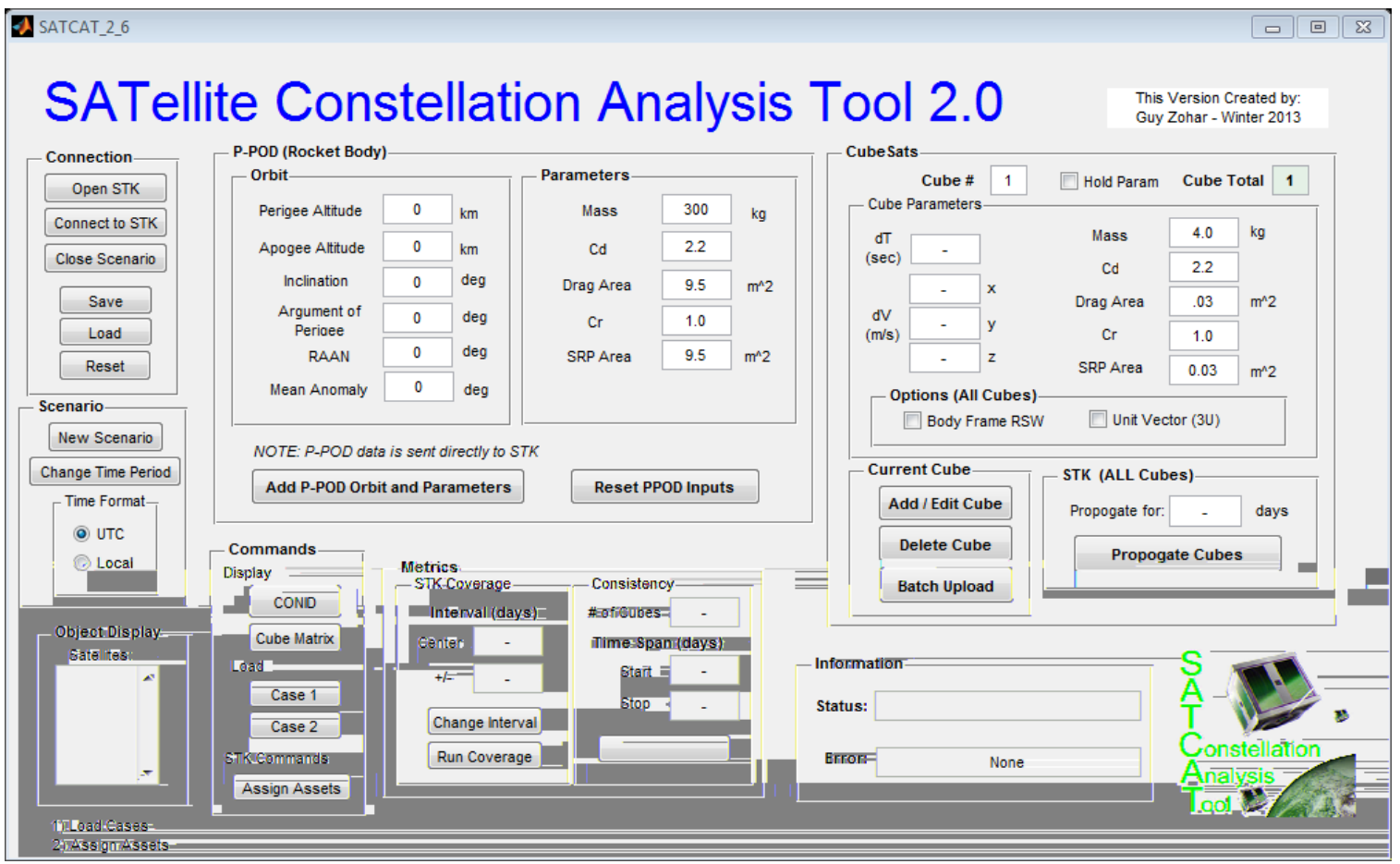

Figure 10: SATCAT 2.0 Graphical User Interface

The P-POD (Rocket Body) module defines the orbit of the rocket upper body that will deploy the CubeSats. The CubeSat module defines the deployment scheme for a user defined variable number of Cubes. This module also allows the user to define satellite properties that are used in STK for accurate propagation. Inside the CubeSat module are the Current Cube and STK (ALL Cubes) modules. The Current Cube module controls the MATLAB GUI portion and allows the user to add, edit, and delete cubes. The STK (ALL Cubes) allows users to select the number of days to propagate the simulation and begin the analysis in STK.

SATCAT 2.0 also allows a user to apply two options to the Cubes being deployed. The Unit Vector (3U) option allows a user to command SATCAT such that the input $\Delta \mathrm{V}$ is a unit vector. SATCAT uses the inputted mass and the data in Table 2 to determine a separation magnitude. Further information about this selection can be found in section 3.1. The Body Frame (RSW) option allows the user to deploy all CubeSats with the relative velocity referencing the body frame, specifically the RSW frame, as opposed to the inertial frame. The RSW frame, also 
referred to as Local Veridical Local Horizontal (LVLH), is centered at the satellite with the $\mathrm{R}$ axis pointing from the Earth's center along the radius vector towards the satellite and shown in Figure 11 below. The $\mathrm{S}$ axis points in the direction of the velocity vector and is perpendicular to the radius vector. The $\mathrm{W}$ axis is normal the orbital plane ${ }^{[9]}$. In SATCAT 2.0 when using the Body Frame $(R S W)$ option, $\mathrm{X}$ is the $\mathrm{R}$ axis, $\mathrm{Y}$ is the $\mathrm{S}$ axis, and $\mathrm{Z}$ is the $\mathrm{W}$ axis.

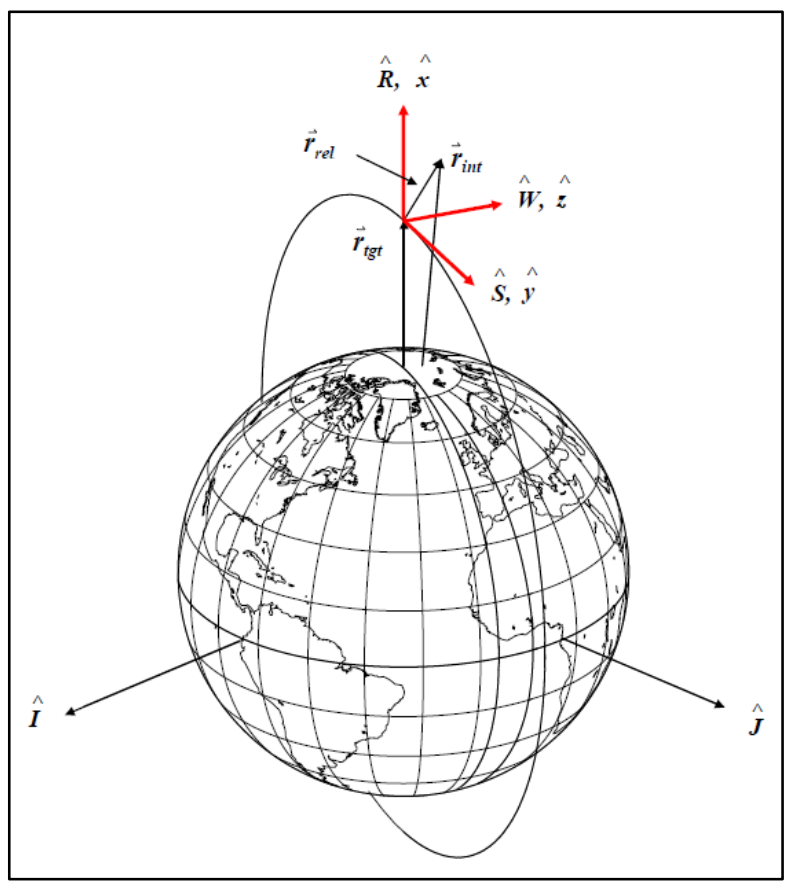

Figure 11: RSW Frame ${ }^{[13]}$

Once the appropriate data is passed into STK, the STK engine propagates the Rocket Body and deploys the appropriate number of Cubes in accordance to the scheme defined in SATCAT. Section 2.3 will detail how STK is set up when running in conjunction with SATCAT 2.0

The Command and Metrics modules are critical in post propagation analysis. The Command module allows the user to display the STK connection ID and the details of the cubes being analyzed. It also allows the user to load Case 1 and 2 into STK and to perform a feature called "Assign Assets". The Metrics module allows the user to command STK regarding coverage and consistency. The STK Consistency sub-module allows the user to change the coverage analysis 
interval and run a coverage analysis in STK. The Consistency sub-module allows the user to command SATCAT and STK to calculate the consistency and Clusterness metrics.

\subsubsection{Using SATCAT 2.0 for CubeSat deployment}

For instructions on how to use SATCAT 2.0 please see Appendix A.

\subsection{STK}

The Systems Tool Kit by AGI is used heavily in the analysis and requires certain options to be selected in order to use the software properly.

\subsubsection{STK Accuracy}

In order to be sure that STK can be used for these propagations its accuracy must first be tested. Using the NPP launch and the RAX-2 CubeSat described in section 2.1, the STK analysis is compared against the JSPoC provided TLEs. The difference between the two is expressed in the classical orbital elements and shown in Figure 12 below.

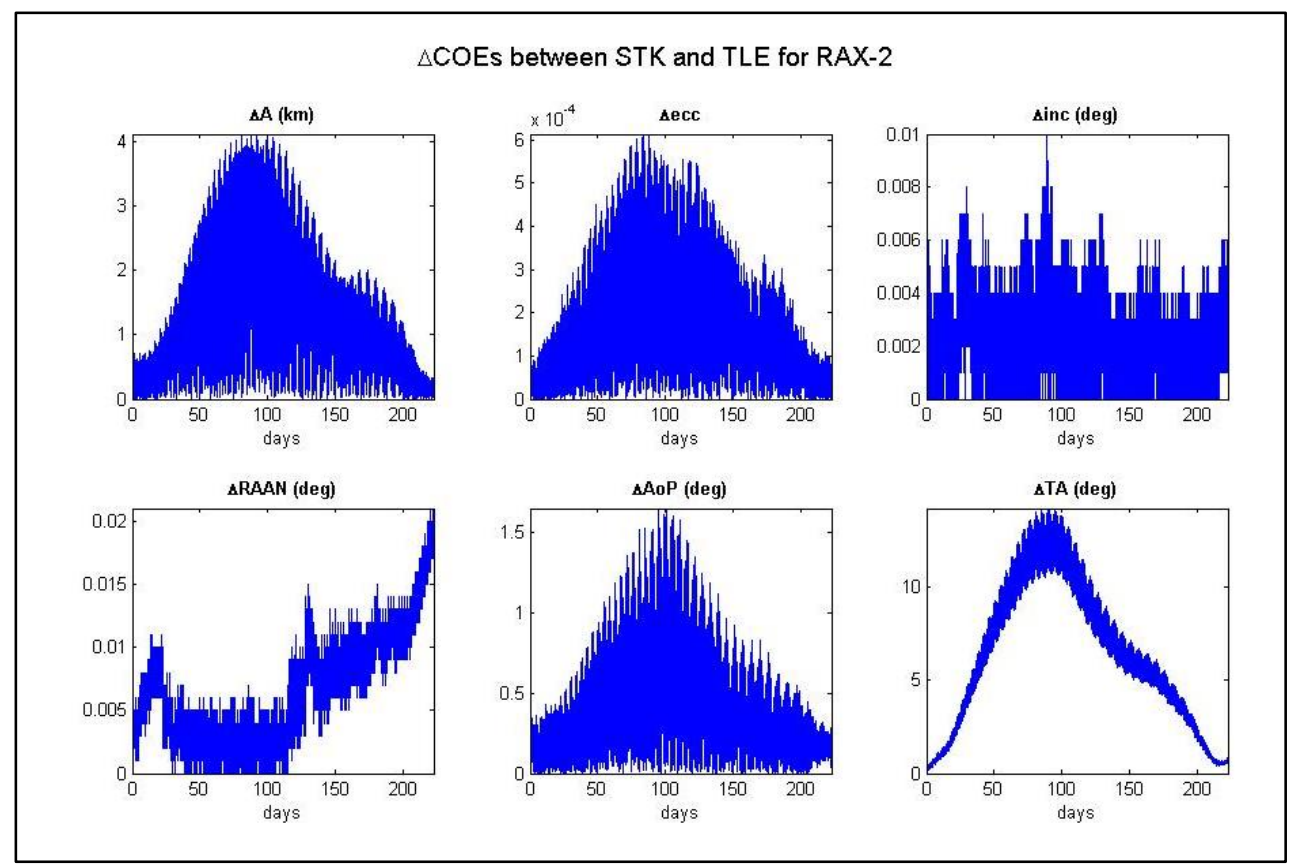

Figure 12: Error between STK analysis and TLEs for RAX-2 
The error in the classical orbital elements is relatively low in all but true anomaly. In semi-major axis, eccentricity, inclination, RAAN, and Argument of perigee the STK analysis is sufficiently accurate over 200 days. The true anomaly error maxes out at approximately $15^{\circ}$ and is caused by the unknown errors in drag discussed in section 2.1.2. This error will be found in all of the satellites analyzed in the same way, resulting in their relative errors being sufficiently low.

\subsubsection{STK Settings}

For use in SATCAT 2.0, STK is set to propagate using the High Precision Orbital Propagator feature with a 30 second time step. The default settings for this propagator are set with modifications on the drag, central body gravity, and solar radiation pressure options.

\subsubsection{Drag}

To compute drag STK inputs an Area over Mass ratio and drag coefficient as defined in SATCAT 2.0. STK also uses NRLMSISE 2000 when being commanded by SATCAT with Space Weather Flux data coming from NOAA.

\subsubsection{Earth Oblateness}

Earth Oblateness is accounted for using the WGS84 EGM96 gravity shape model and utilizing a 15 degree by 15 order level of precision.

\subsubsection{Solar Radiation Pressure (SRP)}

To account for SRP, STK inputs an Area over Mass ratio and reflectivity coefficient from the inputs in SATCAT 2.0. The default setting for this perturbation is to model SRP as spherical with a dual cone shadow of the Earth.

\subsection{Relative Motion Equations (CW - Hill approximations)}

The Hill Equations, also known as the Clohessy-Wiltshire Equations, express the relative motion between two satellites, referred to as a chief and deputy. These equations assume that the chief is 
circular and offer a linearization of the more accurate relative motion equations. However; these assumptions allow for a closed form solution for the relative position and velocity given as ${ }_{[9]}$ :

$$
\begin{gathered}
x(t)=\frac{\dot{x_{o}}}{w} \sin (w t)-\left(3 x_{o}+\frac{2 \dot{y}_{o}}{w}\right) \cos (w t)+\left(4 x_{o}+\frac{2 \dot{y}_{o}}{w}\right) \\
\mathrm{y}(\mathrm{t})=\left(6 \mathrm{x}_{\mathrm{o}}+\frac{4 \dot{\mathrm{y}}_{\mathrm{o}}}{\mathrm{w}}\right) \sin (\mathrm{wt})+\frac{2 \dot{\mathrm{x}}_{\mathrm{o}}}{\mathrm{w}} \cos (\mathrm{wt})-\left(6 \mathrm{wx}_{\mathrm{o}}+3 \dot{\mathrm{y}}_{\mathrm{o}}\right) \mathrm{t}+\left(\mathrm{y}_{\mathrm{o}}-\frac{2 \dot{\mathrm{x}}_{\mathrm{o}}}{\mathrm{w}}\right) \\
z(t)=z_{o} w \sin (w t)+\frac{\dot{z}_{o}}{w} \sin (w t) \\
\dot{x}(t)=\dot{x}_{o} \cos (w t)+\left(2 w x_{o}+2 \dot{y}_{o}\right) \sin (w t) \\
\dot{y}(t)=\left(6 w x_{o}+4 \dot{y}_{o}\right) \cos (w t)-2 \dot{x}_{o} \sin (w t)-2 \dot{x}_{o} \sin (w t)-\left(6 w x_{o}+3 \dot{y}_{o}\right) \\
\dot{z}(t)=-z_{o} w \sin (w t)+\dot{z} \cos (w t)
\end{gathered}
$$

where $\mathrm{x}, \mathrm{y}$, and $\mathrm{z}$, are relative position in the LVLH frame, $\dot{x}, \dot{y}$, and $\dot{z}$ are relative velocity in the LVLH coordinate frame, $w$ is the angular rate of the chief satellite, and $t$ is time.

It is important to note that based on these equations the motion of the deputy relative to the chief satellite in the x-axis and z-axis is oscillatory. As time moves forward the deputy's motion in these axes is bound by sine and cosine terms. However; the motion in the y-axis grows based on the time dependent term shown in equations ( 4 ) and ( 7 ).

In long propagations the error from linearization and orbital perturbations precludes the relative motion equations from being used to accurately predict the relative location of a satellite. However; the trends that the equations describe still hold.

\subsection{Metrics}

To accurately compare multiple jettison profiles results a quantitative method must be utilized. In order to do so specific metrics must be defined and declared. For constellation development of a 
CubeSat two kinds of metrics are useful; the resulting constellations coverage over a given area and how evenly the constellation is dispersed.

\subsubsection{Constellation Coverage}

Two metrics are useful for understanding how well a constellation provides coverage to a given region; coverage time and revisit time. Both metrics are analyzed in STK but and can be commanded either in STK directly or using SATCAT 2.0. Nonetheless, to create contour plots of the regions STK must be used.

STK calculates these metrics based on a given region (Case 1 and 2) which it divides into grid based sub-regions. The grid is determined by latitude and longitude degree step sizes and is set in this analysis to 0.25 degrees; meaning that each analyzed grid square is $0.25 \times 0.25$ degrees of latitude and longitude.

\subsubsection{Coverage Time}

The coverage time metric provides the time a constellation covers an area within the given zone. A high and consistent value is considered a better result as it indicates that the constellation provides more and even coverage time to the zone in question.

\subsubsection{Revisit Time}

The revisit time metric provides the time interval when a region does not have coverage; also known as the gaps. STK can determine this metric in a variety of way; however, the selected option is determining the metric based on the Average revisit time. This option computes the average revisit time of each gap by calculating the length of each gap and dividing by the number of gaps. For this metric a low and consistent number is considered a better result as it indicates that the constellation has minimal time intervals where it does not cover a region. A constellation's ideal value for this metric is zero as it would indicate that no coverage gaps exist. Although the metric being analyzed is the average, it is only the average for each grid point. 
Statistical measures can still be found using the entire region to find the regions maximum, minimum, average, etc.

\subsubsection{Constellation Dispersion}

Two metrics are used to describe how dispersed a constellation is. Clusterness is a "point in time" metric; while Consistency is an interval based metric.

\subsubsection{Clusterness}

Clusterness is a metric created to analyze how evenly satellites are distributed from a single PPOD and is custom created for this thesis. Clusterness is defined between 0 and 1 , where satellites are at maximum separation will result in a metric value of 0 and no separation will result in 1 . While it is trivial to visually compare a 0 and a 1 it is much harder to compare clusters of Cubes in states between the two extremes.

In Figure 13 below, two scenarios are shown that are visually difficult to determine which is more evenly distributed. The satellites in the case on the left are more closely clustered but none of the satellites are extremely close to one another. In the case on the right the satellites are largely distributed over the orbit however two of the satellites are effectively in the same position.

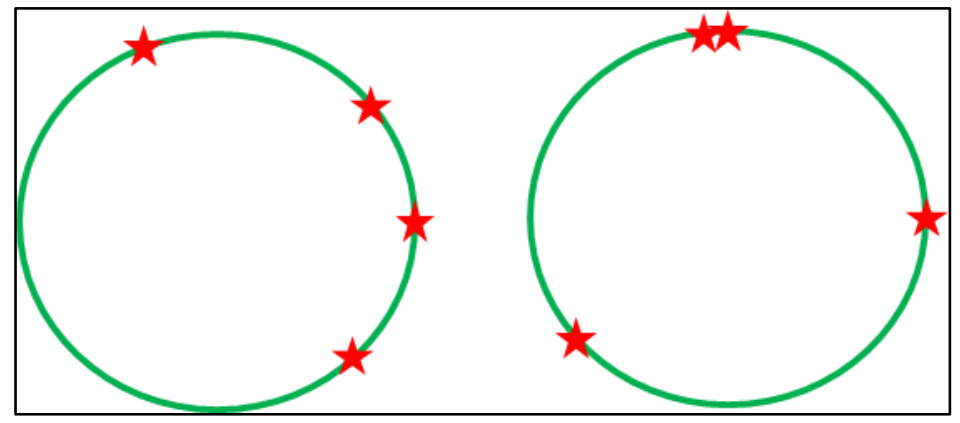

Figure 13: Two cases with varying Clusterness

In order to compare the distribution of the two cases in Figure 13, Clusterness is defined in Equation ( 9 ) below: 


$$
\text { Clusterness }=\frac{\left\|\sum \theta_{\max }-\theta_{N}\right\|}{(N-1) \theta_{\max }}
$$

where $\mathrm{N}$ is the number of satellites, $\theta_{N}$ is the true anomaly separation between satellite $\mathrm{N}$ and $\mathrm{N}+$ 1, and $\theta_{\max }$ follows:

$$
\theta_{\max }=\frac{360}{N}
$$

Using these definitions the left case in Figure 13 has a Clusterness value of 0.3704 with the right case having a Clusterness value of .1667 . These values indicate that the right case is considered less clustered. Even though two satellites are right next to each other the right hand case covers more of the entire orbit.

\subsubsection{Consistency}

Because Clusterness is a moment-in-time metric the "Consistency" metric was developed to track Clusterness over time. Consistency is reported as a graph of Clusterness versus time as shown in Figure 14 below. However, it can also be reported as the maximum minus the minimum Clusterness in a given time frame. This difference shows that over a given time a constellation has changed from one level of Clusterness to another.

As in Figure 14, it can be seen that maximum value is approximately 0.85 with a minimum value of approximately 0.05 . If this number was reported in the other fashion it would be reported as a consistency value of 0.8 over 90 days. This point value method for consistency reporting can be used in analysis methods that require a single point value such as certain Monte Carlo analyses. However; this method does not show when the maximum or minimum are and hides any variations over time and for this reason the graph reporting method is used to study the development of the constellations over time. 


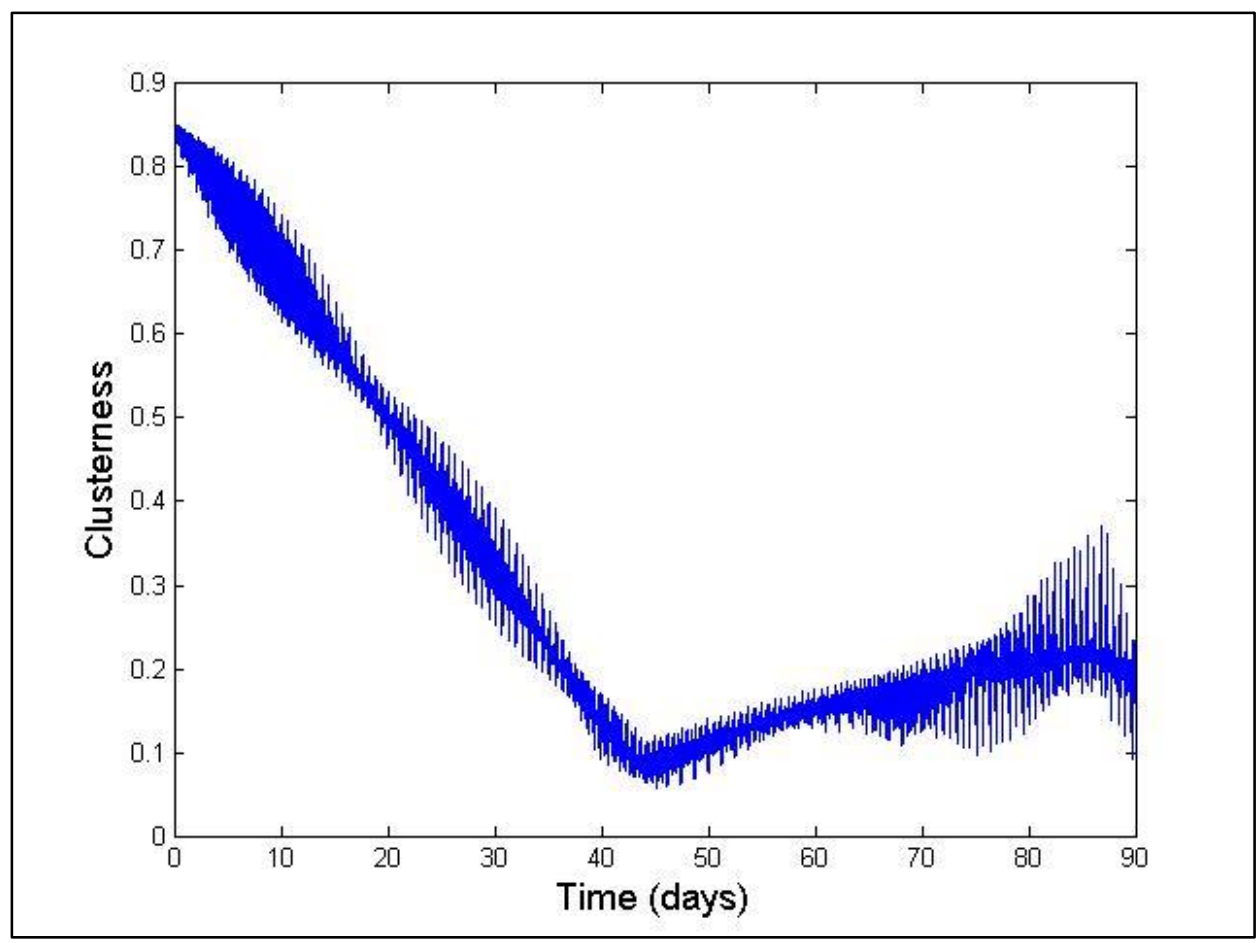

Figure 14: Example Consistency graph 


\section{Critical Analysis}

Before results can be generated SATCAT 2 must be validated and theoretical separation profiles must be analyzed to ensure that the expected results are returned. SATCAT 2 is validated using data from NROL-36 and tested against four theoretical separation profiles.

\subsection{Validation Using NROL-36 Deployment}

NROL-36 is a launch of a classified government satellite that occurred in September 2012 from Vandenberg AFB, CA, using an Atlas V launch vehicle. Onboard was the NPSCul with 8 PPODS and 11 CubeSats.

The CubeSats onboard are as shown in Table 1 below and come in a variety of sizes from multiple organizations. Table 1 also lists the P-POD number that the Cube was in and in which order it was deployed from the rocket body.

Table 1: NROL-36 Cubes ${ }^{[14]}$

\begin{tabular}{|c|c|l|l|c|c|}
\hline $\begin{array}{c}\text { P-POD } \\
\text { Number }\end{array}$ & $\begin{array}{c}\text { Deploy } \\
\text { Number }\end{array}$ & \multicolumn{1}{|c|}{ Satellite Name } & Organization & Size & Image \\
\hline 1 & 1 & $\begin{array}{l}\text { SMDC-ONE 1.2 } \\
\text { (Baker) }\end{array}$ & $\begin{array}{l}\text { US Army } \\
\text { SMDC }\end{array}$ & $3 \mathrm{U}$ & \\
\hline 2 & 8 & $3 x$ AeroCube 4.0 & $\begin{array}{l}\text { Aerospace } \\
\text { Corp }\end{array}$ & $\begin{array}{l}1 \mathrm{U} \text { each } \\
3 \mathrm{U} \text { total }\end{array}$ & $\begin{array}{l}\text { University of } \\
\text { Southern } \\
\text { California }\end{array}$ \\
3 & 2 & Aeneas & $3 \mathrm{U}$ & \\
\hline
\end{tabular}




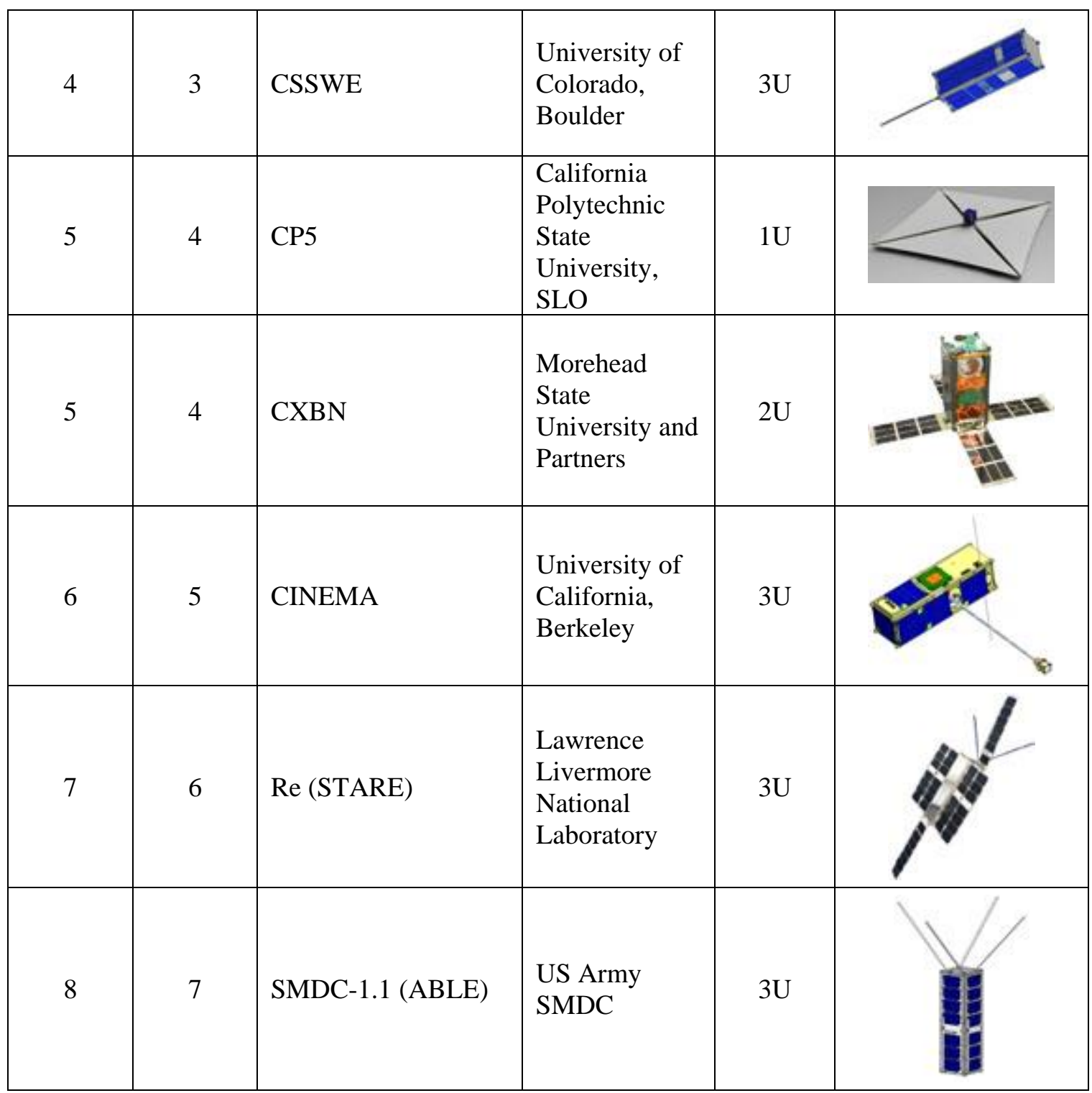

The Cubes from NROL-36 are inputted into SATCAT 2 and propagated forward in STK. The specific mass, area, and other propagation factors for each Cube is proprietary and can be obtained from CubeSat or the specific cube organization. ULA provided Atlas V flight data at each Cube separation event in order to determine the Cube separation vector and time. However, 
the vector provided is for the Centaur not the NPSCul and P-PODs ${ }^{1}$. The NPSCul is installed with an approximate 17 degree offset from the Centaur center-line which must be accounted for as depicted in Figure 15 below.

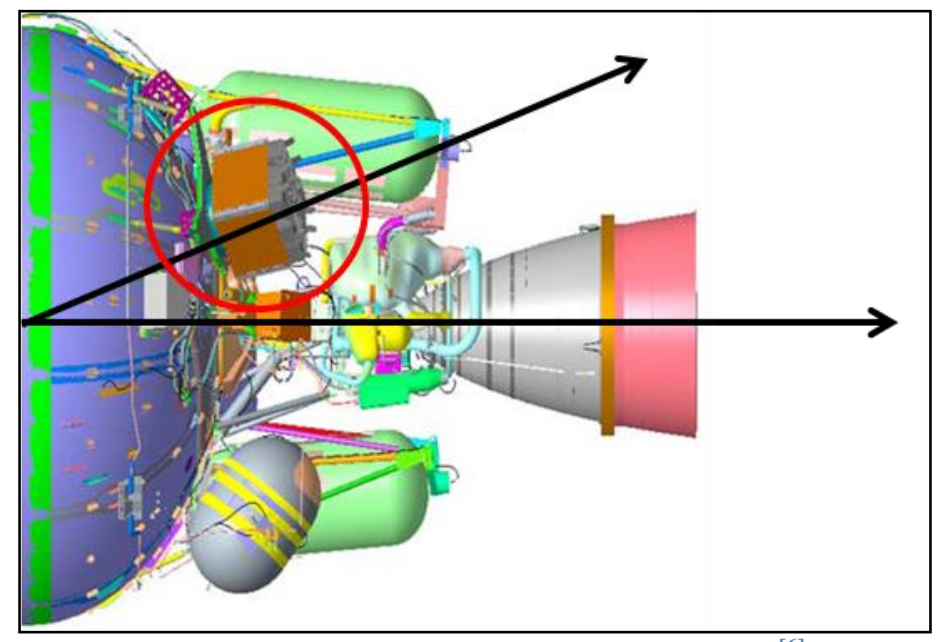

Figure 15: Centaur Bulkhead and NPSCul ${ }^{[6]}$

In order to use the Atlas $\mathrm{V}$ flight data the attitude must be adjusted with a coordinate transformation to account for the NPSCul position. After applying the NPSCul coordinate transform the Atlas V attitude flight data can be used as the unit vector for the CubeSat deployment. The separation velocity magnitude is determined by the P-POD spring and the satellite mass. CubeSat provided separation speed data for a $3 \mathrm{U}$ CubeSat, shown in Table 2 below, from which the specific separation magnitude is linearly interpolated for. The data in Table 2 is the nominal data with an approximately $+/-15 \%$ deviation due to manufacturing of the spring.

\footnotetext{
${ }^{1}$ The specific Atlas V flight data and NPSCuL - Atlas V coordinate transformation is ULA and CubeSat proprietary information and will not be presented in this publication. Contact ULA or CubeSat for this information
} 
Table 2: 3U Cube - PPOD separation speed for given mass

\begin{tabular}{|c|c|}
\hline Mass (kg) & $\begin{array}{c}\text { Separation Speed } \\
(\mathbf{m} / \mathbf{s})\end{array}$ \\
\hline 3 & 1.66 \\
\hline 4 & 1.44 \\
\hline 5 & 1.29 \\
\hline
\end{tabular}

A linear interpolation is appropriate due to the nature of the energy in a spring and the conservation of energy. By setting the potential energy to the kinetic energy and solving for velocity, the velocity of a mass leaving the spring can be expressed as:

$$
V=x \sqrt{\frac{k}{m}}
$$

where $x$ is the compressed spring distance, $k$ is the spring constant, and $m$ is mass. While this relation is not linear for changing mass it can be linearized with limited error for the range of the changing mass shown in Table 2. A linear trend line can be seen in Figure 16 below with a coefficient of determination of 0.9882

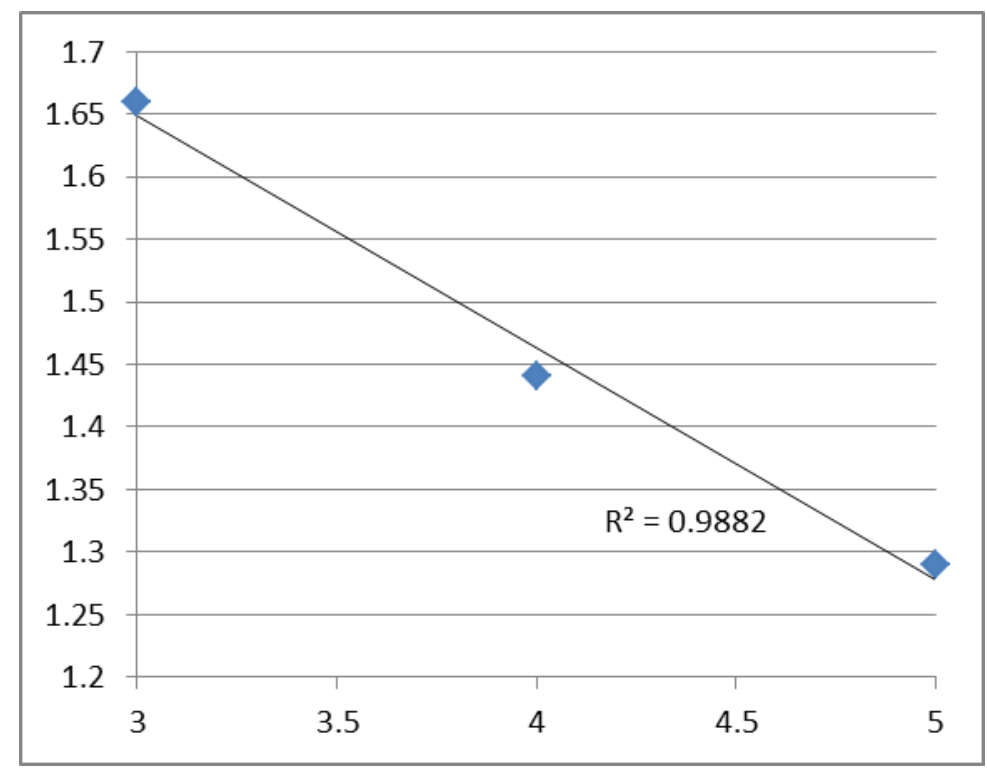

Figure 16: Linear Application of P-POD separation data

With this information the Cube separation velocity vector (both direction and magnitude) is determined. The separation timing is provided in the Atlas V flight data at separation event. This 
information, along with the Cube mass, area, $\mathrm{Cd}$, and $\mathrm{Cr}$ is inputted into SATCAT 2 and propagated.

In order to validate the simulation "true" values must be used to compare against. For this purpose the JSpOC generated TLE's are used to validate the model. An STK feature allows for representation of each of the CubeSat's TLE as an STK object. Using this feature each Cubes simulation is compared via classical orbital elements to its TLE in STK. As the simulation assumes a $3 \mathrm{U}$ satellite only the $3 \mathrm{U}$ satellites are compared.

A comparison of the classical orbital elements of each of the 3U CubeSat's from the NROL-36 mission is found in Appendix B. As seen in Appendix B there are small shifts in the true anomaly error in the results of AENEAS, CSSWE, SMDC_1.1, and SMDC_1.2. This shift is present in the raw TLE data and not in the simulation data and is present due to the classification and reporting system that JSpOC uses to report TLE's. When the satellites are in close proximity occasionally TLE's of two or more Satellites can become swapped for a short period of time. This can cause a shift in the TLE that must be accounted for. The small jump shifts present in Appendix B are artifacts of this correction.

\subsubsection{Potential Error Sources}

The results in Appendix B are mixed. For SMDC 1.1, SMDC 1.2, and AENEAS the error is relatively low; however, the CSSWE, Re, and CINEMA have a large error. As discussed in section 2.1.1 the simulation models each Cube as a $3 \mathrm{U}$ cube with fixed cross sectional area. Errors in both groups of Cubes can be attributed to incorrect model assumptions.

SMDC 1.1 and 1.2 closely resemble the assumption and their errors are lowest in the analysis. While the image of AENEAS in Table 1 shows AENEAS as having a large antenna dish, this was not deployed until after the analysis time. Due to the lack of deployment of the antenna dish AENEAS also fits the simulation assumptions. The error shown for SMDC 1.1, SMDC 1.2, and 
AENEAS can be attributed to small deviations between the assumed and true cross sectional area and coefficient of drag.

CINEMA, Re, and CSSWE have much larger errors. These errors are associated with large deviations between the simulation assumptions and true parameters. CINEMA has a large boom used for attitude control, Re has large deployable solar panels, and CSSWE does not hold a constant cross sectional area has an average cross sectional area lower than the simulation assumption value. Changing the drag effect on each of these satellites should reduce the error. CINEMA's drag is increased by 3.5 times due to the size of the boom, Re is increased by 3.0 times, and CSSWE is reduced by a factor of 0.5. Using the updated assumptions in Appendix B show the errors associated between the simulated CINEMA, Re, and CSSWE versus the reported TLEs.

\subsection{Theoretical Deployments}

After validating the simulation against NROL-36 a series of theoretical Cube deployment schemes are tested as a benchmark. Some of the deployment schemes would be extremely difficult to execute; however, these scenarios would likely never be implemented and serve to bound the design space.

Each deployment scheme is initialized on 1 Jan 2012 at 07:00:00 UTC and propagated for 90 days and later examined. Further each scenario is deployed from a 500 x $800 \mathrm{~km}$ orbit with an inclination of $45^{\circ}$. Argument of perigee, RAAN, and true anomaly are initially set to $0^{\circ}$.

\subsubsection{Cubes At Varying Time With No $\Delta V$}

The first scenario deploys 11 Cubes at varying times, shown in Table 3, with a separation velocity of $0 \mathrm{~m} / \mathrm{s}$. While a separation velocity of $0 \mathrm{~m} / \mathrm{s}$ cannot feasibly be executed, this scenario serves to show how cubes can be kept in a cluster and not separated over time. 
Table 3: Varying deployment time with $\Delta V=0 \mathrm{~m} / \mathrm{s}$

\begin{tabular}{|c|c|c|}
\hline Cube & $\begin{array}{c}\text { Time } \\
(\mathbf{m i n})\end{array}$ & $\begin{array}{c}\text { Time } \\
(\mathbf{s e c})\end{array}$ \\
\hline 1 & 0 & 0 \\
\hline 2 & 1 & 60 \\
\hline 3 & 2 & 180 \\
\hline 4 & 5 & 300 \\
\hline 5 & 10 & 600 \\
\hline 6 & 15 & 900 \\
\hline 7 & 30 & 1800 \\
\hline 8 & 60 & 3600 \\
\hline 9 & 90 & 5400 \\
\hline 10 & 120 & 7200 \\
\hline 11 & 180 & 10800 \\
\hline
\end{tabular}

This deployment has a consistency output shown in Figure 17 below.

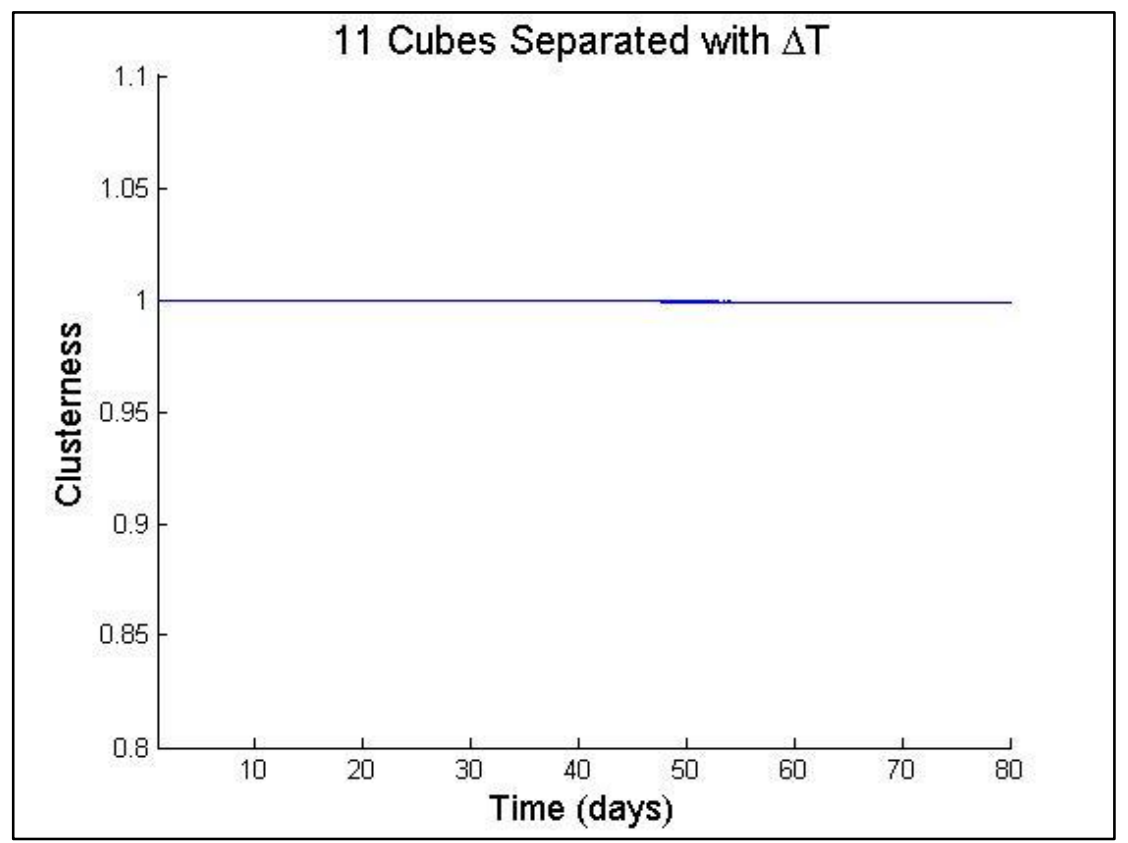

Figure 17: Consistency Output for 11 Cubes with varying $\Delta T$

The 11 Cubes do not separate from one another over the 80 days. An extremely small deviation from a Clusterness value of 1.0 is reached after 50 days. 
This result is the expected result; given a $\Delta \mathrm{V}$ of $0 \mathrm{~m} / \mathrm{s}$ all Cubes will stay with the rocket body as it continues to separate new cubes. While this separation velocity is unfeasible the analysis shows that an extremely low $\Delta \mathrm{V}$ will likely result in clustered cubes.

\subsubsection{Cubes at varying time with identical $\Delta \mathrm{V}$}

The next deployment scheme uses the same separation times shown in Table 3; however, each cube has a $\Delta \mathrm{V}$ equal to the "nominal" $3 \mathrm{U}$ CubeSat separation speed of $1.5 \mathrm{~m} / \mathrm{s}$. This separation velocity is applied in the negative velocity vector.

This vector was selected as it was the initial believed vector that the Cubes were normally launched in. Further analysis has shown that this scheme was not used in the NROL-36 launched as initially believed. Nevertheless, it represents a separation scheme that many CubeSat developers believe is often used.

Figure 18 below shows the consistency metric output for this deployment scenario.

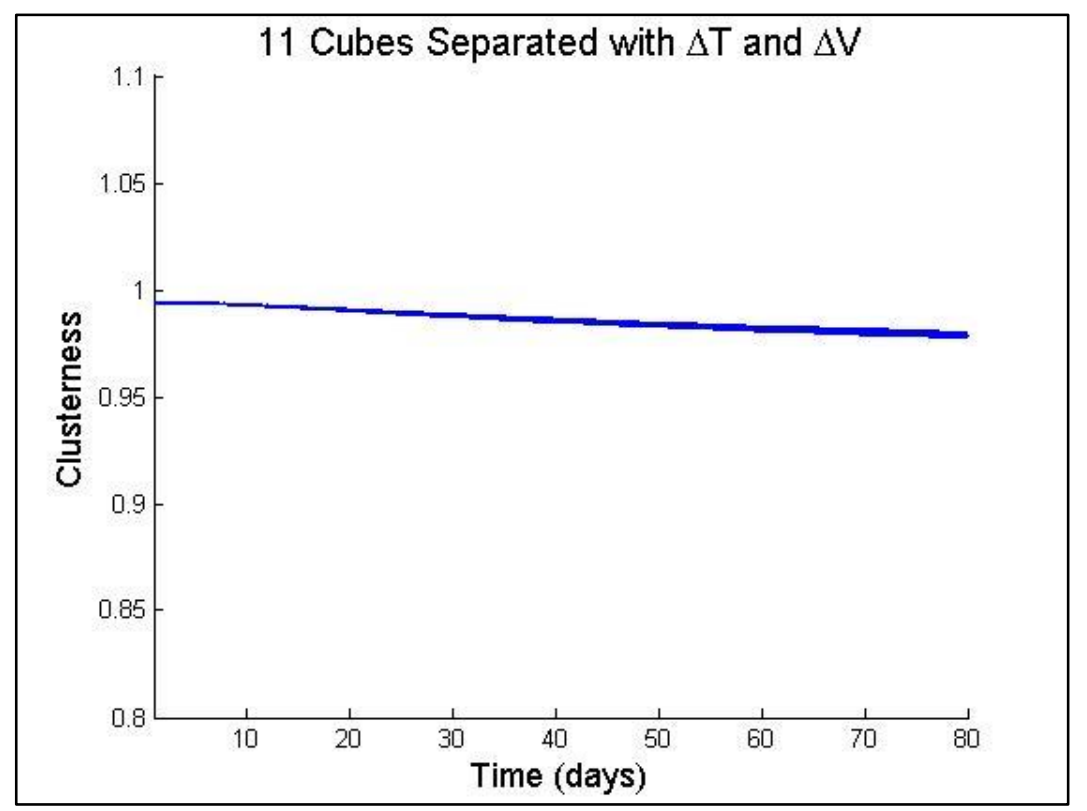

Figure 18: Consistency Output for 11 Cubes with varying $\Delta T$ and $\Delta V=1.5 \mathrm{~m} / \mathrm{s}$

While this scenario does show noticeable separation over 80 days it is very muted. When compared with the $\Delta \mathrm{V}=0$ scheme the cubes distribute further but not significantly so. While the 
previous scenario was also unfeasible to execute this scenario is extremely feasible and could be utilized if the developer's goal is to keep Cubes clustered together.

\subsubsection{RSW Axis Separation}

The third scheme deploys a total of 12 cubes at the same time. Six cubes are separated with relative velocity of $0.5 \mathrm{~m} / \mathrm{s}$ and the other 6 cubes with a relative velocity of $1.5 \mathrm{~m} / \mathrm{s}$ and are separated in the $\mathrm{X}, \mathrm{Y}$, and $\mathrm{Z}$ axes of the RSW coordinate frame; as shown in Table 4 below.

Table 4: RSW axis separation vectors for 12 Cubes

\begin{tabular}{|c|c|c|c|}
\hline \multirow{2}{*}{$\begin{array}{c}\text { Cube } \\
\text { Number }\end{array}$} & \multicolumn{3}{|c|}{ Velocity $(\mathbf{m} / \mathbf{s})$} \\
\cline { 2 - 4 } & $\mathbf{X}$ & $\mathbf{Y}$ & $\mathbf{Z}$ \\
\hline $\mathbf{1}$ & 0.5 & 0 & 0 \\
\hline $\mathbf{2}$ & -0.5 & 0 & 0 \\
\hline $\mathbf{3}$ & 0 & 0.5 & 0 \\
\hline $\mathbf{4}$ & 0 & -0.5 & 0 \\
\hline $\mathbf{5}$ & 0 & 0 & 0.5 \\
\hline $\mathbf{6}$ & 0 & 0 & -0.5 \\
\hline $\mathbf{7}$ & 1.5 & 0 & 0 \\
\hline $\mathbf{8}$ & -1.5 & 0 & 0 \\
\hline $\mathbf{9}$ & 0 & 1.5 & 0 \\
\hline $\mathbf{1 0}$ & 0 & -1.5 & 0 \\
\hline $\mathbf{1 1}$ & 0 & 0 & 1.5 \\
\hline $\mathbf{1 2}$ & 0 & 0 & -1.5 \\
\hline
\end{tabular}

This separation scheme shows what happens to the development of Cubes that are launched in an independent direction without a varying time. It results in a consistency graph shown in Figure 19 below. 


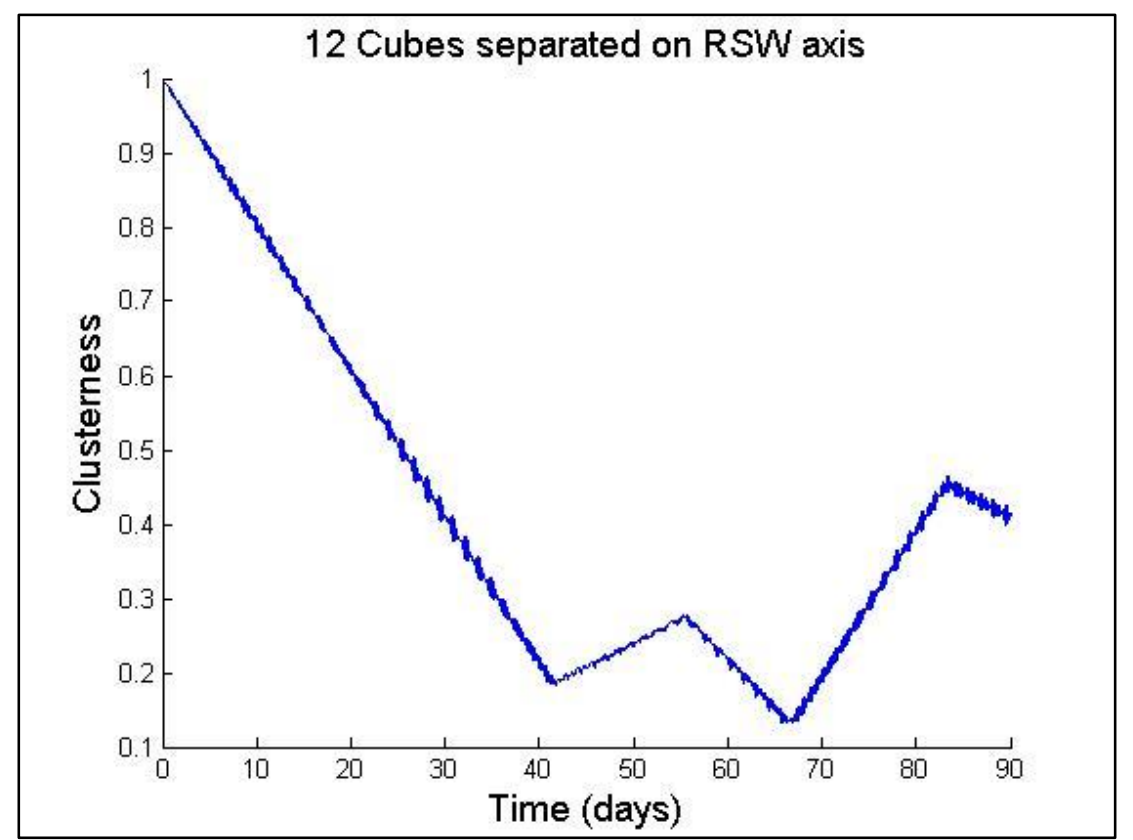

Figure 19: 12 Cubes separated on RSW axis with no time between separations

The consistency graph above shows rapid dispersal of the cubes over the first 40 days follow by a period of oscillation. This oscillation is due to the fact that the constellation has "fully" developed and is beginning to collapse. As shown this process includes many local minima as each development and collapse build on one another. Some developments and collapses can be dramatically different from one another, such as the ones that occur on day 65 and 85 , and will require that potential development schemes be analyzed for a longer time span.

While the consistency output shows that the Clusterness values of the deployment drop down to approximately 0.15 direct examination of the results in STK yield additional information that must be accounted for. Figure 20 shows the simulated orbit positions of the Cubes 65 days into the analysis with 5 sets annotated. Set A consists of Cube numbers 1, 2, 5, 6, 7, 8, 11, and 12. Sets B, C, D, and E consist of Cube numbers 3, 10, 9, and 4 respectively. 


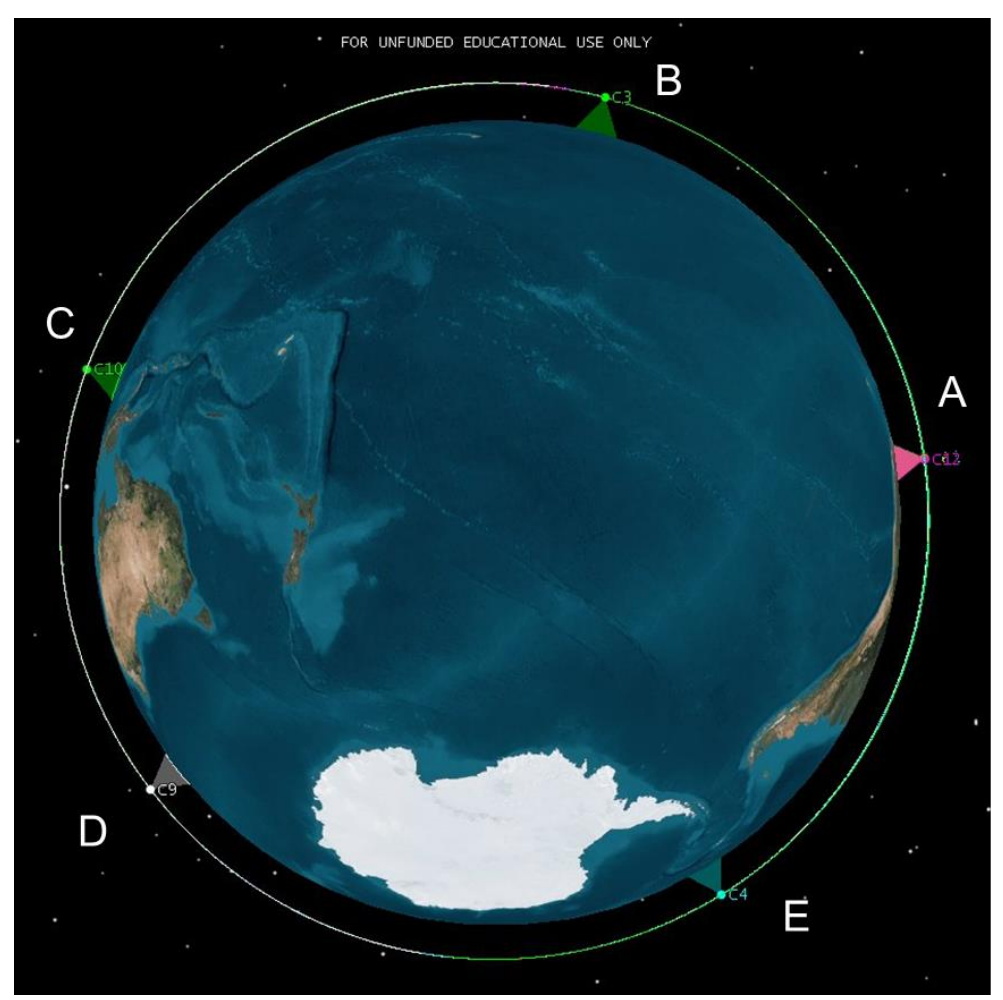

Figure 20: 5 groups of CubeSat clusters

Examination of each group yields further understanding as to the coupling between the independent axes.

As shown in Table 4 the cubes in Set $\mathrm{A}(1,2,5,6,7,8,11$, and 12) all have varying velocities in the $\mathrm{X}$ and $\mathrm{Z}$ directions. Sets $\mathrm{B}, \mathrm{C}, \mathrm{D}$, and $\mathrm{E}$ have varying magnitudes of separation entirely in the $\mathrm{Y}$ axis. Note that regardless of the magnitude of a Cubes separation, if the separation direction is in the $\mathrm{X}$ or $\mathrm{Z}$ axis it does not separate from any other Cube with separation direction in the $\mathrm{X}$ or $\mathrm{Z}$ axis. Further all separation occurs from varying magnitudes in the $\mathrm{Y}$ axis.

The grouping in Set A is also the reason that the Clusterness of the constellation never drops below 0.15. If each set in Figure 20 were an individual Cube then the overall Clusterness value would be significantly closer to 0 . However, based on the definition of Clusterness, show in Equation (9), when multiple cubes are in the same position the value increases. Effectively the deployment scheme is over saturated and only one cube from Set A would be required to obtain a near 0 Clusterness. 
This deployment serves as a strong benchmark for developers wishing to reach a large and even separation between deployed Cubes. Unlike in the previous two separation schemes, in which the Cubes stayed extremely closely together, this scheme allows for, albeit over saturated, near maximum separation.

\subsection{X-Y and Y-Z Planar separation}

Based on the results of the RSW axes separation scheme in section 3.2.3, analysis of separation in the $\mathrm{X}-\mathrm{Y}$ and $\mathrm{Y}-\mathrm{Z}$ planes is conducted. The scheme concluded that cubes with an $\mathrm{X}$ and $\mathrm{Z}$ separation directions would stay together while cubes in a Y direction would separate. However; the scheme did not include analysis on Cubes that had both a Y component and an $\mathrm{X}$ or $\mathrm{Z}$ component. This separation scheme deploys two sets of 8 cubes into the $\mathrm{X}-\mathrm{Y}$ and the $\mathrm{Y}-\mathrm{Z}$ planes, as shown in Table 5 and Figure 21 below. As with the RSW axes separation scheme all cubes are separated at the same time and propagated for 90 days. The Cubes in the X-Y plane are separated with $\mathrm{X}$ and $\mathrm{Y}$ components while the cubes in the $\mathrm{Y}-\mathrm{Z}$ plane are separated with $\mathrm{Y}$ and $\mathrm{Z}$ components.

Table 5: X-Y and Y-Z separation direction based on degree offset from $+Y$

\begin{tabular}{|c|c|c|c|c|c|c|c|c|}
\hline \multirow{3}{*}{$\begin{array}{c}\theta(\mathrm{deg}) \\
\text { separation } \\
\text { from }+\mathrm{Y}- \\
\text { axis } \\
\end{array}$} & \multicolumn{8}{|c|}{ Separation Velocity Vector $(\mathrm{m} / \mathrm{s})$} \\
\hline & \multicolumn{4}{|c|}{ X-Y Plane } & \multicolumn{4}{|c|}{ Y-Z Plane } \\
\hline & Cube & $\mathbf{X}$ & $\mathbf{Y}$ & $\mathbf{Z}$ & Cube & $\mathbf{X}$ & $\mathbf{Y}$ & $\mathbf{Z}$ \\
\hline 0 & $\mathbf{x 1}$ & 0 & 1.44 & 0 & $\mathbf{z 1}$ & 0 & 1.44 & 0 \\
\hline 45 & $\mathbf{x 2}$ & 1.02 & 1.02 & 0 & $\mathbf{z 2}$ & 0 & 1.02 & 1.02 \\
\hline 90 & $\mathbf{x 3}$ & 1.44 & 0 & 0 & $\mathbf{z 3}$ & 0 & 0 & 1.44 \\
\hline 135 & $\mathrm{x4}$ & 1.02 & -1.02 & 0 & $\mathrm{z4}$ & 0 & -1.02 & 1.02 \\
\hline 180 & $\mathbf{x 5}$ & 0 & -1.44 & 0 & $\mathbf{z 5}$ & 0 & -1.44 & 0 \\
\hline 225 & $\mathrm{x6}$ & -1.02 & -1.02 & 0 & $\mathrm{z6}$ & 0 & -1.02 & -1.02 \\
\hline 270 & $\mathbf{x 7}$ & -1.44 & 0 & 0 & $\mathbf{z 7}$ & 0 & 0 & -1.44 \\
\hline 315 & $\mathbf{x 8}$ & -1.02 & 1.02 & 0 & $\mathbf{z 8}$ & 0 & 1.02 & -1.02 \\
\hline
\end{tabular}




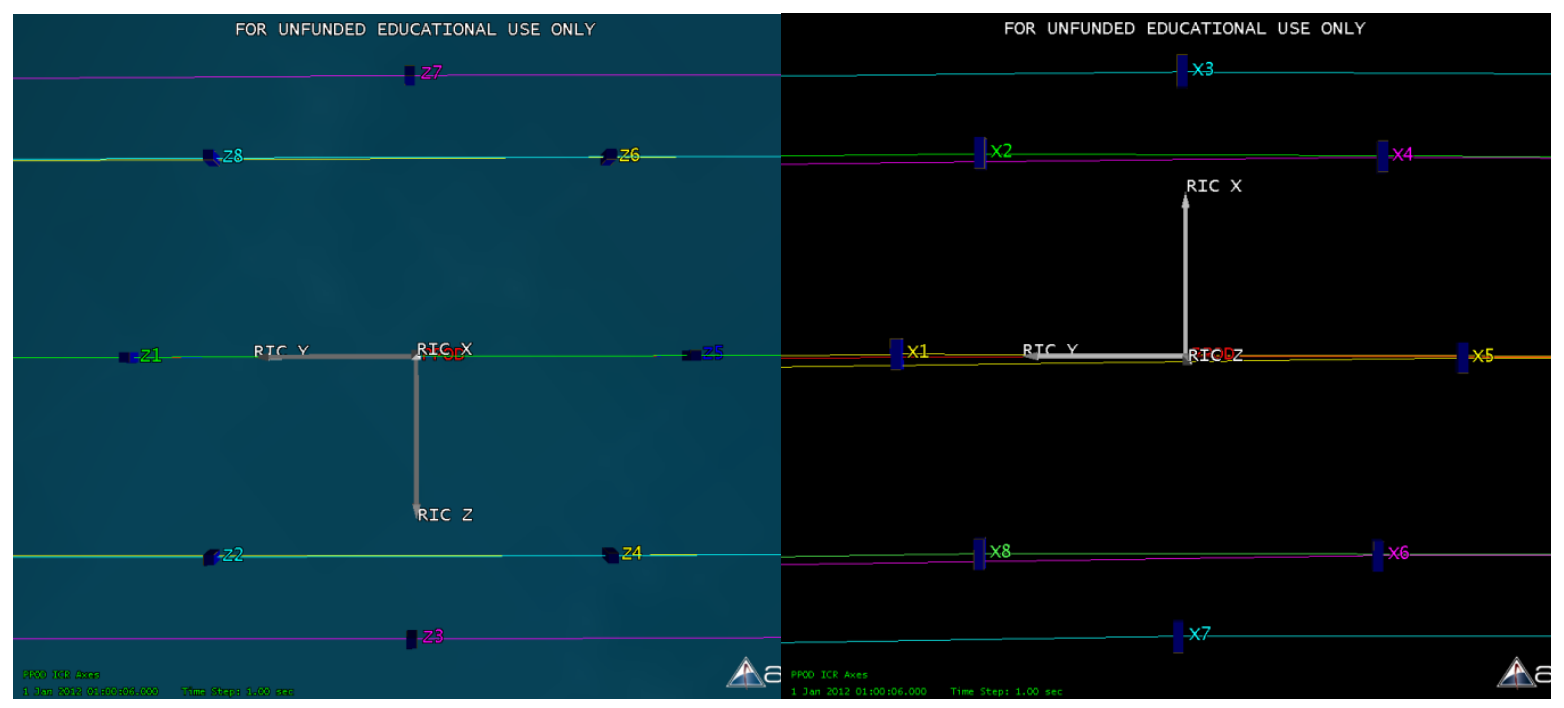

Figure 21: Separation directions in $\mathrm{X}-\mathrm{Y}$ and $\mathrm{Y}-\mathrm{Z}$ planes

This separation scheme is deployed from a 500 x 550 orbit with an inclination of 51.6 degrees starting on 1 Jan 2012 01:00:00 UTC. Argument of Perigee, RAAN, and True Anomaly are set to zero. Figure 22 below shows the consistency of each plane over the propagation time. Each plane has an identical consistency over the 90 days examined.
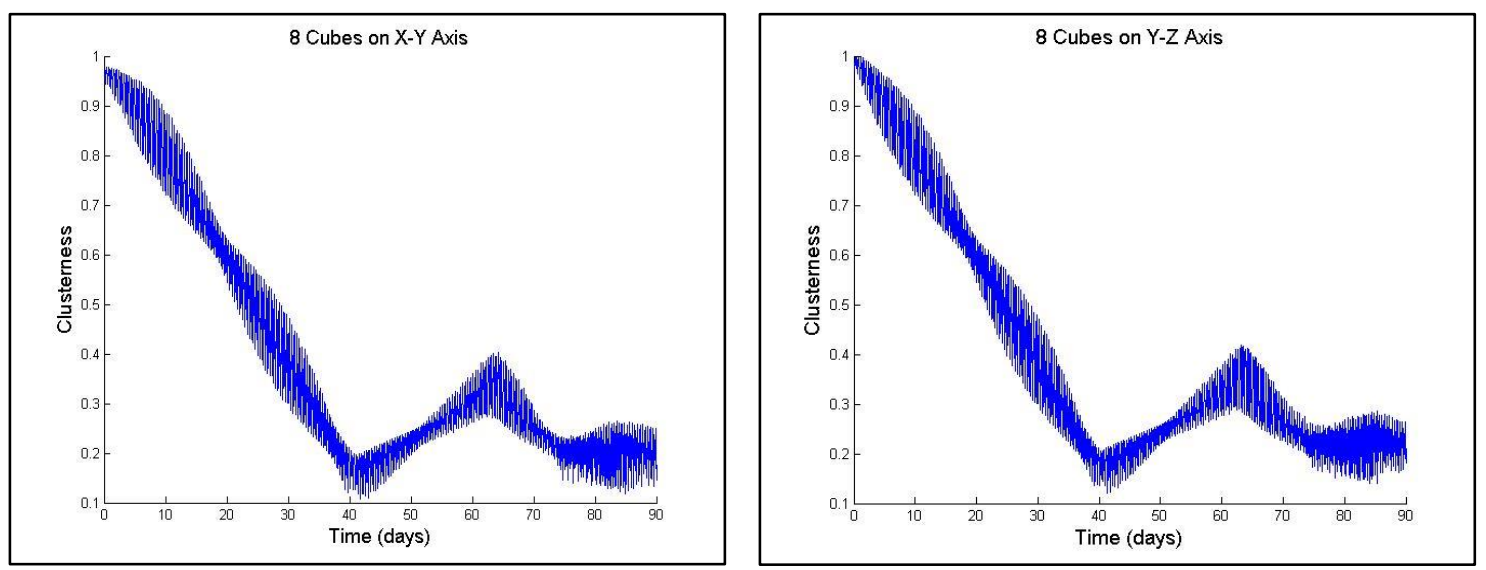

Figure 22: Consistency for separation in $\mathrm{X}-\mathrm{Y}$ and $\mathrm{Y}-\mathrm{Z}$ planes

When directly examined the Cubes cluster into 5 groups with varying numbers of Cubes in each.

Table 6 below denotes the Cubes in each group. Group A and E each include two cubes; however as shown in Table 5, these cubes have identical separation velocity vectors. Groups B, C, and D each include the four cubes that share the same separation in the Y vector. For example group B includes all the cubes in the separation scheme that have a Y vector component equal to $1.02 \mathrm{~m} / \mathrm{s}$. 
Table 6: X-Y and Y-Z plane separation Cube grouping

\begin{tabular}{|c|c|c|}
\hline Group & Cubes in Group & Y component (m/s) \\
\hline A & $\mathrm{x} 1, \mathrm{z} 1$ & 1.44 \\
\hline B & $\mathrm{x} 2, \mathrm{z} 2, \mathrm{x} 8, \mathrm{z} 8$ & 1.02 \\
\hline C & $\mathrm{x} 3, \mathrm{z} 3, \mathrm{x} 7, \mathrm{z} 7$ & 0 \\
\hline D & $\mathrm{x} 4, \mathrm{z} 4, \mathrm{x} 6, \mathrm{z} 6$ & -1.02 \\
\hline E & $\mathrm{x} 5, \mathrm{z} 5$ & -1.44 \\
\hline
\end{tabular}

The grouping conforms to the trend analysis of the relative motion equations, discussed in Section 2.4; as the long term location of the cube does not depend on the $\mathrm{X}$ or $\mathrm{Z}$ vectors. Only the $\mathrm{Y}$ vector affects the long term location of the satellite. In each group the X-Y plane cubes oscillate back and forth along the $\mathrm{X}$ axis while $\mathrm{Y}-\mathrm{Z}$ plane cubes oscillate along the $\mathrm{Z}$ axis.

By varying the direction of separation into the $\mathrm{X}$ or $\mathrm{Z}$ plane the $\mathrm{Y}$ component can be changed because the magnitude of separation is fixed. This separation scheme shows that to obtain noticeable separation only the $\mathrm{Y}$ component is a design factor. 


\section{Results}

In order to determine the best separation schemes for Case 1 and 2 further analysis is required. Analysis of a single plane in a nominal orbit shows the effects the separation geometry on constellation Clusterness. Analysis on cube separation from specific launch sites provides detail on how each launch correlates to coverage time of Case 1 and 2. Analysis on multiple planes provides detail on augmenting a constellation from a single plane with one or more launches, and its effect on the coverage time of Case 1 and 2.

Based on the $\mathrm{X}-\mathrm{Y}$ and $\mathrm{Y}-\mathrm{Z}$ plane separation scheme in Section 3.3, only a variation in the $\mathrm{Y}$ component will result in Cube separation. Hence all further separations will be conducted in the $\mathrm{X}-\mathrm{Y}$ plane only.

\subsection{Single Plane Analysis}

Analysis in the X-Y plane is done from a 500 x 550 orbit at a $51.6^{\circ}$ inclination with an Argument of Perigee, RAAN, and True anomaly all set to zero. All propagations are initialized on 1 Jan 2012 01:00:00 UTC. Further, each launch supports 8 Cubes per plane as this the maximum number of $3 \mathrm{U}$ cubes the NPSCuL can support per launch. As mentioned in section 2.1.1, each cube is modeled as a $4 \mathrm{~kg} 3 \mathrm{U}$ CubeSat. Based on this mass, each Cube separated from the PPOD with a speed of $1.44 \mathrm{~m} / \mathrm{s}$

\subsubsection{Angle based Semi-Circle Separation (X-Y plane)}

Initially a semi-circle deployment scheme in the X-Y plane is analyzed with a cube being released every 3 minutes in equal increment along the semi-circle, starting from $+\mathrm{Y}$ and ending at -Y. This scheme allows for each cube to have a unique separation in the Y-axis and is an efficient attitude maneuver for a rocket body to perform. This scheme is propagated for 365 days in order to understand the long term effects of the deployment. Table 7 and Figure 23 below depict the 
separation scheme. Note that Figure 23 shows all Cubes separating at one time to clarify the deployment scheme; however, the cubes are actually separated on the time schedule in Table 7.

Table 7: Angle based deployment times and direction

\begin{tabular}{|c|c|c|c|c|c|}
\hline \multirow{2}{*}{ Cube } & \multirow{2}{*}{ Time (min) } & \multirow{2}{*}{$\Theta(\mathbf{d e g})$ from $+\mathbf{Y}$} & \multicolumn{3}{|c|}{ Separation Vector $(\mathbf{m} / \mathbf{s})$} \\
\cline { 4 - 6 } & & 0 & $\mathbf{X}$ & $\mathbf{Y}$ & $\mathbf{Z}$ \\
\hline 1 & 0 & 25.714 & 0.62 & 1.44 & 0 \\
\hline 2 & 3 & 51.428 & 1.13 & 0.90 & 0 \\
\hline 3 & 6 & 77.142 & 1.40 & 0.32 & 0 \\
\hline 4 & 9 & 102.857 & 1.40 & -0.32 & 0 \\
\hline 5 & 12 & 128.571 & 1.13 & -0.90 & 0 \\
\hline 6 & 15 & 154.285 & 0.62 & -1.30 & 0 \\
\hline 7 & 18 & 180 & 0 & -1.44 & 0 \\
\hline 8 & 21 & & & & \\
\hline
\end{tabular}

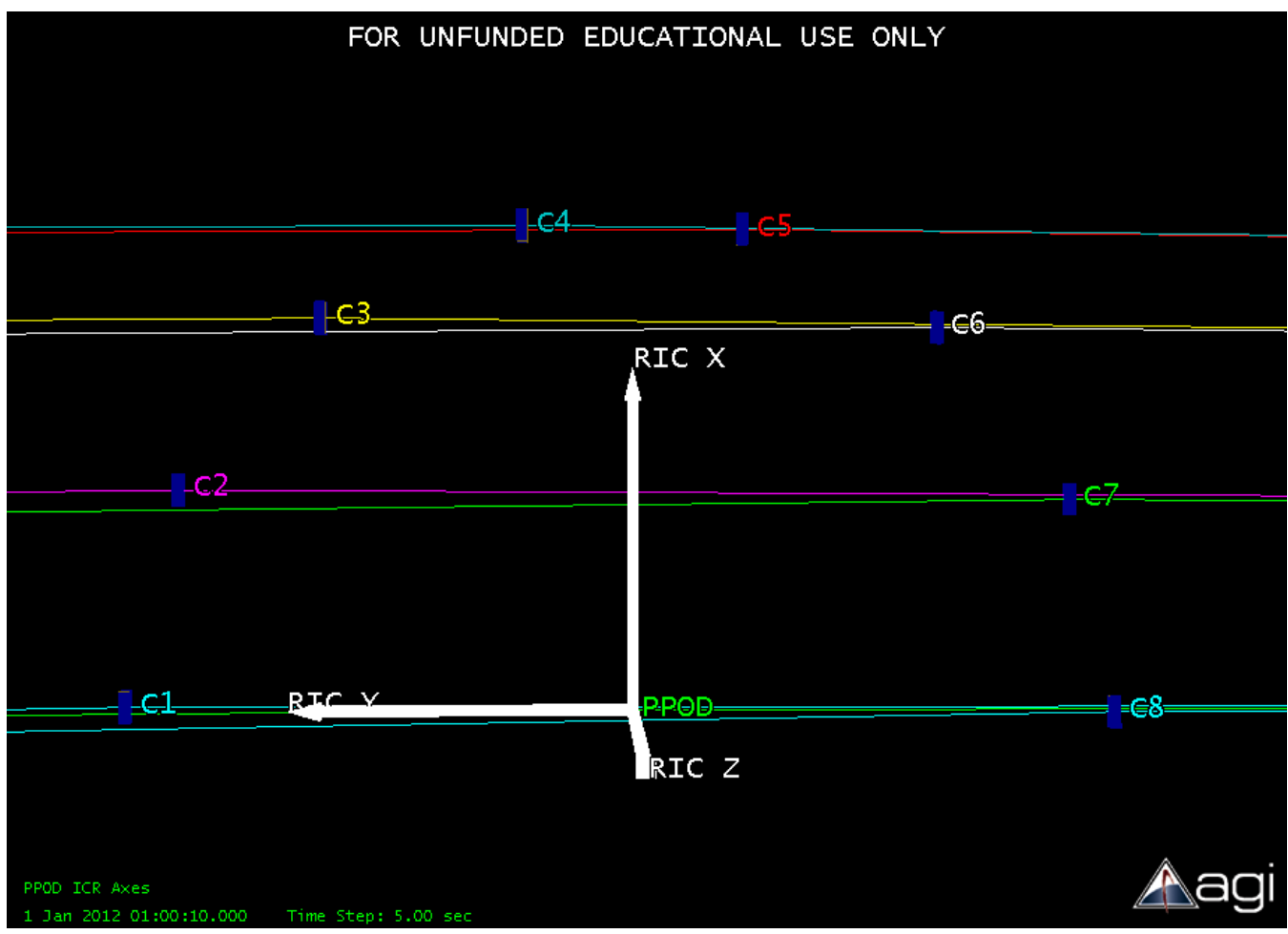

Figure 23: Superposition of 8 Cubes separated in semi-circle scheme

After 365 days the consistency output is shown in Figure 24 below. The scheme results in the cubes in the constellation separating quickly and reaching a near minimum Clusterness value after approximately 45 days. The developed constellation then oscillates above the near minimum value by breaking down and reforming periodically but does not reach a state significantly less clustered. 
The absolute minimum value over the year occurs at 203 days with a Clusterness value of 0.0513 . Forty-five days after separation from the P-POD, the constellation has developed to a Clusterness value of 0.0614 while the absolute minimum Clusterness value does not occur until significantly later in the orbital lifetime of the constellation. A CubeSat developer is unlikely to wait 158 days for a Clusterness change of only 0.0101 . Due to the small gain obtained by waiting 158 days, the time the constellation reaches its initial deployment is considered the time required to deploy the constellation.

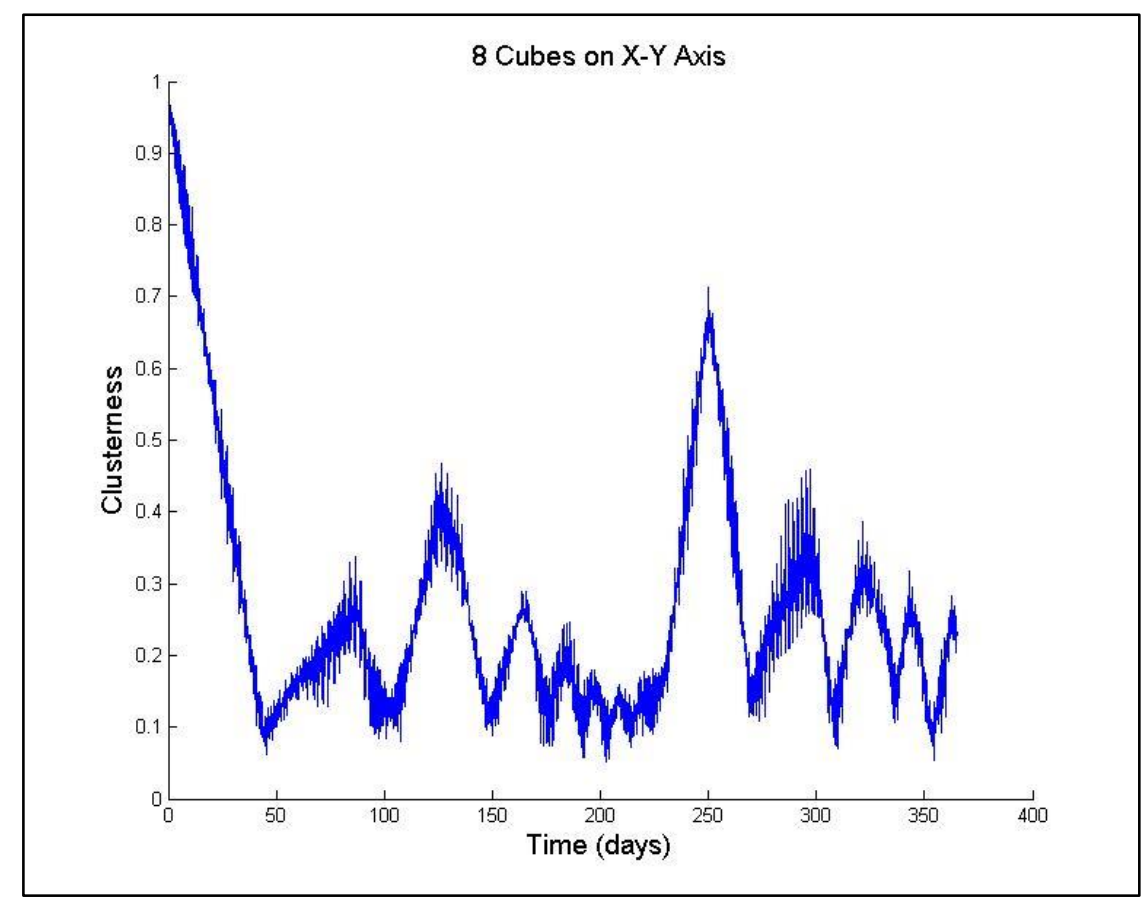

Figure 24: Angle based semi-circle separation consistency

Over the year the constellation fluctuates between a Clusterness value of approximately 0.05 and 0.7. The cubes regularly reach their Clusterness near the minimum value and re-cluster; however, throughout the year there are some dramatic spikes in Clusterness which last approximately 50 days.

Examining the constellation directly at its initial maximum separation on day 45 shows which cubes are closer to others; resulting in a non-zero Clusterness value. As shown in Figure 25 
below, the cubes are distributed along the orbit in the order they were dispersed and subsequently in the order of their separation in the Y-vector. The Y-vector between each cube is not constant and this varying difference is proportional to the separation found in Figure 25.

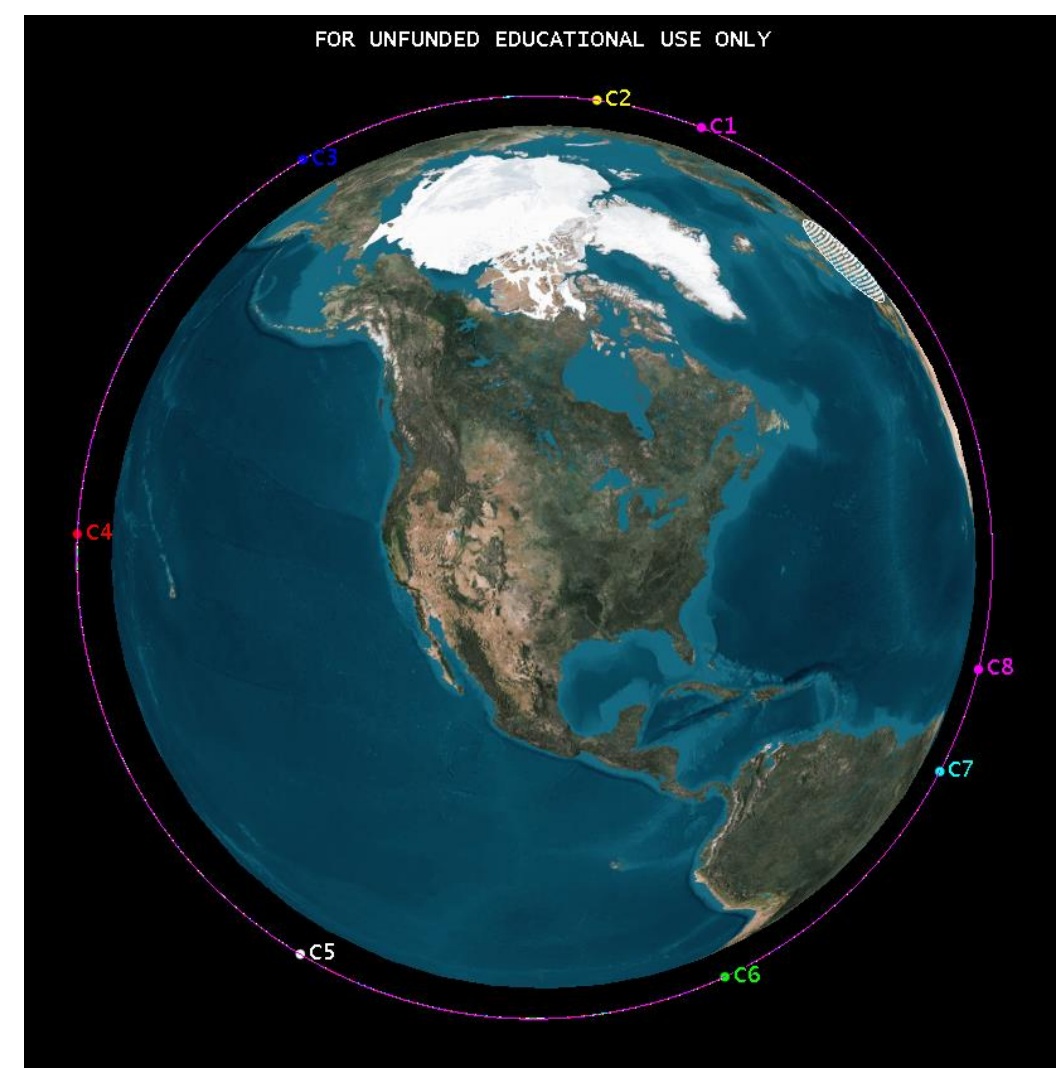

Figure 25: Simulated cube positions after 45 days from semi-circle spacing

The cubes are separated evenly on the semi-circle by angle thus the resulting Y-vector is not equally separated. For example the $\Delta \mathrm{V}$ between Cube 1 and 2 is $0.14 \mathrm{~m} / \mathrm{s}$ while the $\Delta \mathrm{V}$ between Cube 2 and 3 is $0.4 \mathrm{~m} / \mathrm{s}$. As expected, Figure 25 confirms that Cubes 1 and 2 are closer together than Cubes 2 and 3. It also shows that Cubes 7 and 8 are separated by the same distance as Cubes 1 and 2; this is also expected because the $\Delta \mathrm{V}$ between Cubes 7 and 8 is the same as $\Delta \mathrm{V}$ between Cube 1 and 2. In fact all the cubes follow this same pattern with the cubes launched in the $+Y$ direction mirroring the results of those in the $-\mathrm{Y}$ direction.

Due to this effect, equal separation based on the Y-vector would theoretically yield a lower Clusterness value. This separation scheme is discussed in section 4.1.2. 


\subsubsection{Varying Altitude (effect on COE's and consistency)}

Examining the semi-circle separation scheme at varying altitudes is a critical measure for CubeSat developers. Due to the uncertain launch opportunities for CubeSats a developer must understand how deployment orbits at different altitudes translate into constellation consistency. In the original deployment scheme the orbit selected was a 6928 x $6878 \mathrm{~km}$ radius $(500 \times 550 \mathrm{~km}$ altitude) with a 51.6 degree inclination. To ensure that only the effect of altitude is examined eccentricity held constant while the perigee radius is varied between 6778 and $7028 \mathrm{~km}$; based on a variation between 400 and $650 \mathrm{~km}$ altitude and Earth's radius of $6378 \mathrm{~km}$. Using the initial $6928 \times 6878 \mathrm{~km}$ radius the eccentricity is held at 0.003621 . Ten perigee radii equally spaced between 6778 and $7028 \mathrm{~km}$ are examined and shown in Table 8 below.

Table 8: Varying altitudes using angle-based semi-circle deployment

\begin{tabular}{|c|c|c|c|c|c|}
\hline $\begin{array}{c}\text { Perigee } \\
\text { Point \# }\end{array}$ & $\begin{array}{c}\text { Perigee } \\
\text { Radius (km) }\end{array}$ & $\begin{array}{c}\text { Apogee } \\
\text { Radius }(\mathbf{k m})\end{array}$ & $\begin{array}{c}\text { Perigee } \\
\text { Altitude } \\
(\mathbf{k m})\end{array}$ & $\begin{array}{c}\text { Apogee } \\
\text { Altitude } \\
(\mathbf{k m})\end{array}$ & $\begin{array}{c}\text { Semi-major } \\
\text { Axis (km) }\end{array}$ \\
\hline $\mathbf{1}$ & 6778.0 & 6827.3 & 400 & 449.3 & 6802.6 \\
\hline $\mathbf{2}$ & 6805.8 & 6855.2 & 427.8 & 477.2 & 6830.5 \\
\hline $\mathbf{3}$ & 6833.6 & 6883.2 & 455.6 & 505.2 & 6858.4 \\
\hline $\mathbf{4}$ & 6861.3 & 6911.2 & 483.3 & 533.2 & 6886.3 \\
\hline $\mathbf{5}$ & 6889.1 & 6939.2 & 511.1 & 561.2 & 6914.1 \\
\hline $\mathbf{6}$ & 6916.9 & 6967.2 & 538.9 & 589.2 & 6942 \\
\hline $\mathbf{7}$ & 6944.7 & 6995.1 & 566.7 & 617.1 & 6969.9 \\
\hline $\mathbf{8}$ & 6972.4 & 7023.1 & 594.4 & 645.1 & 6997.8 \\
\hline $\mathbf{9}$ & 7000.2 & 7051.1 & 622.2 & 673.1 & 7025.7 \\
\hline $\mathbf{1 0}$ & 7028.0 & 7079.1 & 650 & 701.1 & 7053.5 \\
\hline
\end{tabular}

As discussed in section 4.1.1 only the initial deployment value is relevant to a CubeSat developer and thus the initial deployment value and time are the compared metrics which are shown in Table 9 and Figure 26 below. 
Table 9: Deployment time and Clusterness due to varying altitude

\begin{tabular}{|c|c|c|}
\hline Perigee Alt & $\begin{array}{c}\text { Time to full } \\
\text { deployment (days) }\end{array}$ & Clusterness \\
\hline 400 & 38.48 & 0.05388 \\
\hline 427.8 & 42.18 & 0.05812 \\
\hline 455.6 & 44.1 & 0.05633 \\
\hline 483.3 & 44.91 & 0.05723 \\
\hline 511.1 & 45.52 & 0.06062 \\
\hline 538.9 & 46.81 & 0.05819 \\
\hline 566.7 & 47.16 & 0.05613 \\
\hline 594.4 & 47.52 & 0.05836 \\
\hline 622.2 & 47.72 & 0.05742 \\
\hline 650 & 48.83 & 0.05902 \\
\hline
\end{tabular}

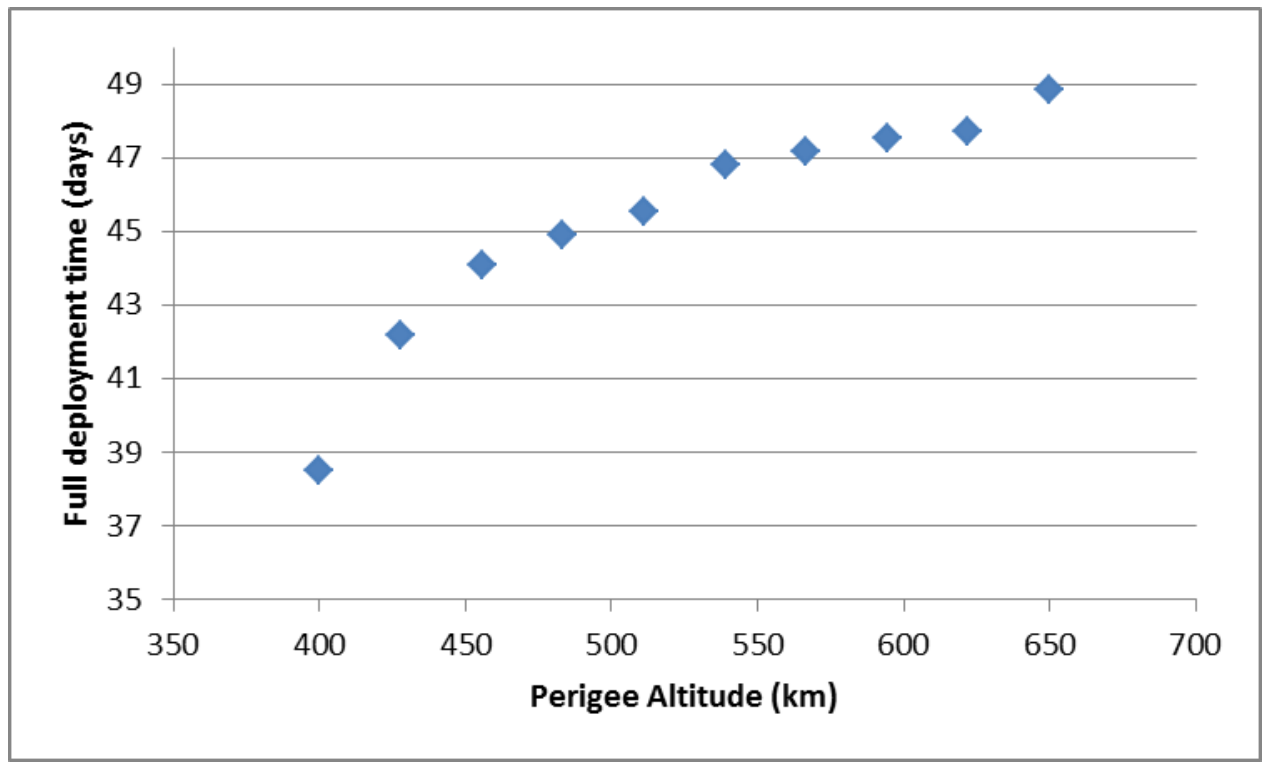

Figure 26: Varying perigee altitude vs. time to full deployment

It is clear that an increased perigee altitude results in a longer time for full deployment; however, there is a negligible effect on the minimum Clusterness value. As perigee altitude increases from 400 to $650 \mathrm{~km}$ the time required for full deployment increases from 38 to 50 days. Varying the initial obit altitude yields non-linear results due to the non-linear effects of perturbations over time and the trend shown by the Gaussian form of the Variation of Parameters (VoP).

Given the same time period, a scheme that results in larger changes to the true anomaly correlates to a quicker time to full deployment. This is because the cubes in the constellation are drifting in the orbit faster than their counterparts in an orbit which results in a low change in true anomaly. 
Due to the fact that "time to full deployment" is dominated by the orbit's change of true anomaly the VoP equation governing the change in true anomaly over time can be used to explain the trend shown in Figure 26 above and is given as ${ }^{[15]}$ :

$$
\frac{d \theta}{d t}=\frac{h}{r^{2}}+\frac{1}{e h}\left[p \cos \theta f_{r}-(p+r) \sin \theta f_{\theta}\right]
$$

Where $t$ is time, $\theta$ is true anomaly, $h$ is the specific orbital angular momentum, $r$ is the specific radius from the central body, $e$ is eccentricity, $p$ is the semilatus rectum, and $f_{r}$ and $f_{\theta}$ are the forces of perturbation in the radial and circumferential directions respectively. As the altitude and thus radius increases, $\frac{d \theta}{d t}$ decreases due to the dominant $1 / \mathrm{r}^{2}$ term in equation ( 12 ). A decrease in $\frac{d \theta}{d t}$ implies that the Cubes in the constellation are drifting slower and will take longer to reach full deployment. This trend is confirmed by the data in Table 9 .

A change in altitude will affect the length of time a constellation requires to develop but does not affect its minimum Clusterness value.

\subsubsection{Varying $\Delta T$}

Although the initial semi-circle deployment scheme separated a cube every 3 minutes, this time is a variable that can be adjusted to change the development of the deployed constellation. In order to understand the effects that this time variable has on the deployed constellation multiple separation times must be explored. The original relative separation time $(\Delta \mathrm{T})$ of 3 minutes was selected due to its flight heritage on NROL-36 but a design space between 1 and 10 minutes are potential options. A $\Delta \mathrm{T}$ less than 1 minute would not generally be used by a CubeSat developer. A $\Delta \mathrm{T}$ greater than 10 minutes begins to push the total deployment sequence time over 1.5 hours; a generally non-optimum condition for many upper stage rockets.

In order to understand the effects that relative separation time has on the developed constellation the initial $500 \times 550$ orbit, used in section 4.1 is used as the separation orbit. Each simulation 
separates 8 Cubes every $\Delta \mathrm{T}$ minutes, with the profiles shown in Table 7 , and propagated until the constellation reaches initial full deployment. Five relative separations times equally spaced between 1 and 10 minutes are tested and have their associated time to full deployment and minimum Clusterness value recorded in Table 10 below.

Table 10: Constellation development due to increasing separation time; kept equal between cubes

\begin{tabular}{|c|c|c|}
\hline$\Delta \mathbf{T}(\mathbf{m i n})$ & $\begin{array}{c}\text { Time to Full } \\
\text { Deployment (days) }\end{array}$ & Clusterness \\
\hline 1.00 & 46 & 0.0596 \\
\hline 3.25 & 45.81 & 0.05477 \\
\hline 5.00 & 46 & 0.05822 \\
\hline 7.75 & 46.6 & 0.06731 \\
\hline 10.0 & 46.6 & 0.06441 \\
\hline AVEREAGE & $\mathbf{4 6 . 2 0 2}$ & $\mathbf{0 . 0 6 0 8 6 2}$ \\
\hline STD Deviation & $\mathbf{. 3 7 1 5 1}$ & $\mathbf{0 . 0 0 4 9 9 6}$ \\
\hline
\end{tabular}

Based on these results, a constant relative separation time does not significantly affect the time to full deployment or minimum Clusterness value. This only explores different relative separation times that are constants between each cube. To ensure that there isn't an effect from a change of separation time between the cubes in a single a test with an increasing separation time is run.

Table 11 below denotes the times in which each Cube is separated.

Table 11: Varying relative separation time between each Cube separation event

\begin{tabular}{|c|c|c|}
\hline Cube \# & Time (min) & $\boldsymbol{\Delta} \mathbf{T}(\mathbf{m i n})$ \\
\hline 1 & 0 & - \\
\hline 2 & 1 & 1 \\
\hline 3 & 4 & 3 \\
\hline 4 & 9 & 5 \\
\hline 5 & 16 & 7 \\
\hline 6 & 26 & 10 \\
\hline 7 & 31 & 15 \\
\hline 8 & 61 & 30 \\
\hline
\end{tabular}

With this Cube deployment the time to full deployment is 47.71 days and a Clusterness of 0.6287 .

The relative separation time does not significantly affect the time to full deployment or minimum Clusterness value. 


\subsubsection{Equally Distributed Y-Vector Component Based Semi-Circle Separation}

The separation scheme in Section 4.1.1 is equally spaced by angle on the deployment semicircle which causes unequal spacing in the Y-component of the separation. As discussed in section 4.1.1, an uneven distribution in Y-vector separation velocity yields an uneven distribution in the constellation. The separation scheme in this section is based on equally distributed Y-vector components. The $\mathrm{X}$-axis components are then calculated based on the constant separation magnitude of $1.44 \mathrm{~m} / \mathrm{s}$. In order to compare this scheme with the angle based separation the deployment times are identical. The cubes are propagated for 365 days. Table 12 below fully describes the separation scheme.

Table 12: Y-vector based semi-circle deployment times and direction

\begin{tabular}{|c|c|c|c|c|c|}
\hline \multirow{2}{*}{ Cube } & \multirow{2}{*}{ Time (min) } & \multirow{2}{*}{$(\mathbf{d e g})$ from $+\mathbf{Y}$} & \multicolumn{4}{|c|}{ Separation Vector $(\mathbf{m} / \mathbf{s})$} \\
\cline { 4 - 6 } & & $\mathbf{X}$ & $\mathbf{Y}$ & $\mathbf{Z}$ \\
\hline 1 & 0 & 0.00 & 0.00 & 1.44 & 0 \\
\hline 2 & 3 & 44.41 & 1.01 & 1.03 & 0 \\
\hline 3 & 6 & 64.62 & 1.30 & 0.62 & 0 \\
\hline 4 & 9 & 81.79 & 1.43 & 0.21 & 0 \\
\hline 5 & 12 & 98.21 & 1.43 & -0.21 & 0 \\
\hline 6 & 15 & 115.38 & 1.30 & -0.62 & 0 \\
\hline 7 & 18 & 135.59 & 1.01 & -1.03 & 0 \\
\hline 8 & 21 & 180.00 & 0.00 & -1.44 & 0 \\
\hline
\end{tabular}

The consistency results of this separation are shown in Figure 27 below and confirm the initial trend discussed in section 4.1.1. 


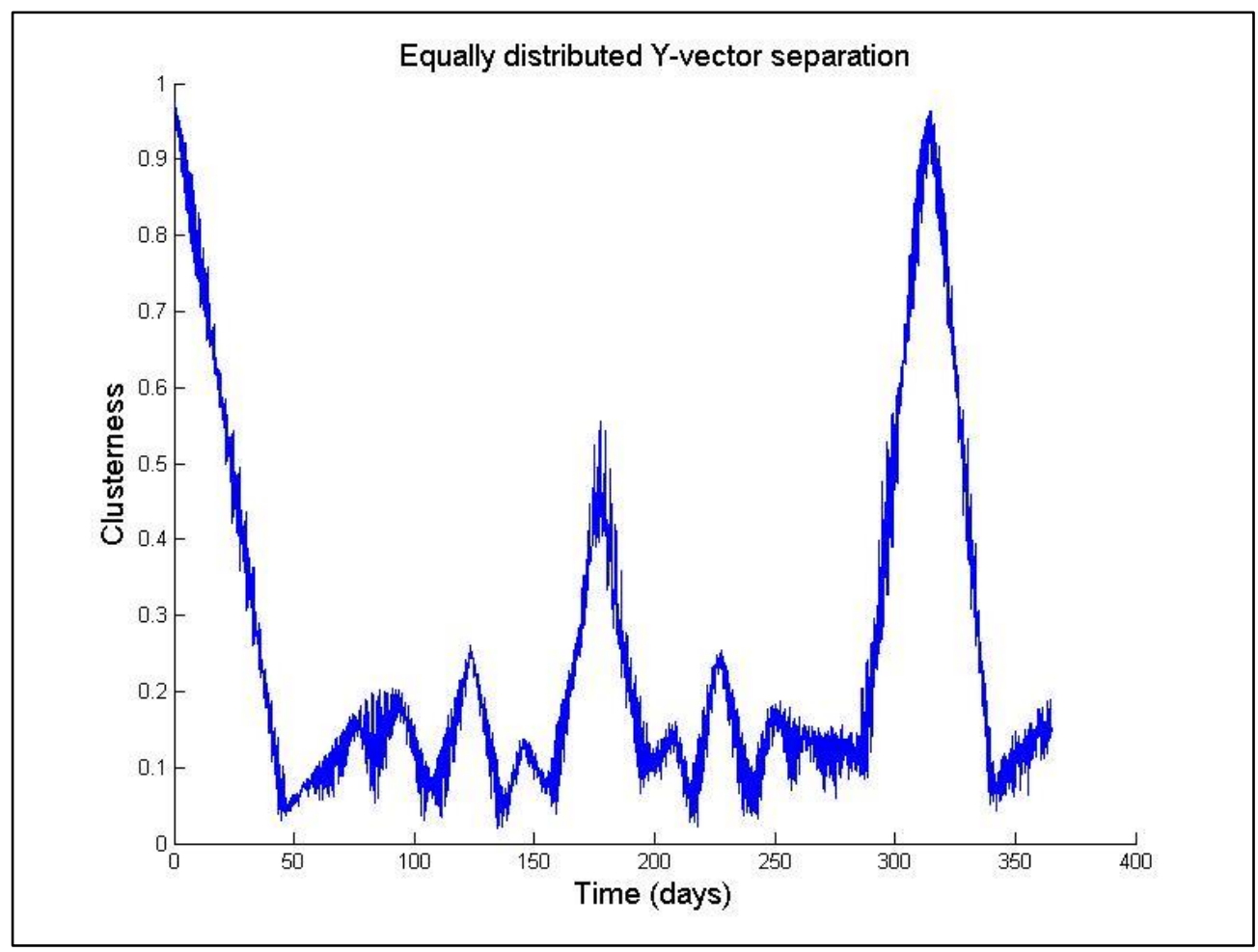

Figure 27: Equal Y-vector semi-circle separation consistency

The minimum Clusterness value of the constellation is 0.02099 with the initial full development reaching a Clusterness value of 0.02967 after 44.93 days. The simulated position of each Cube in the constellation on day 45 is shown in Figure 28 below. 


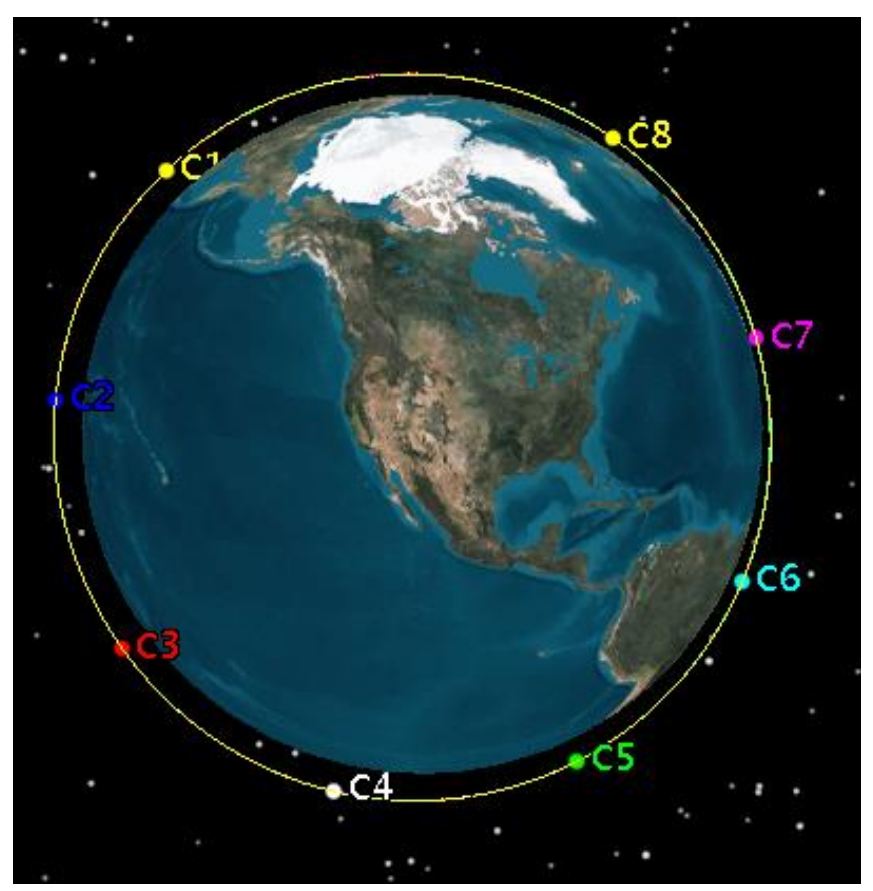

Figure 28: Simulated cube positions after 45 days from equally distributed $Y$-vector separation

Similar to the results of the separation based on angle-based semi-circle spacing this separation scheme oscillates over time and has two peaks where the constellation is highly clustered. This separation has peaks that are more dramatic and reach approximately 0.6 and 1.0 and the oscillations are closer to zero. These dramatic peaks might make this separation profile undesirable for certain CubeSat developers. This scheme is more likely to be utilized by a constellation developer planning on performing an orbital maneuver to stop the constellation from breaking down.

A separation based on equally distributed $\mathrm{Y}$-vector separation velocities and applied to a semicircle geometry produces a significantly non-clustered constellation. However; this maneuver is not as optimal for the rocket body as it must rotate a different angle before each separation. In the angle-based separation the rocket body would be able to repeat a single maneuver until all the cubes are separated. 


\subsection{Constellation Break Down}

Each deployed constellation naturally breaks down and builds back up in an oscillatory fashion. Multiple examples of this breakdown are presented in sections 3.2 and 4.1. This oscillatory pattern is not optimal for many constellation operators. If nothing is done to mitigate this drift, the constellation will continue to oscillate for the lifetime of the system.

Depending on the deployment scheme the developed constellation will break apart at different rates and to different extents. As shown in Figure 24, the angle based separation s builds up in approximately 45 days to its minimum Clusterness value of approximately 0.1 . However, it collapses it almost as rapidly as it developed and oscillates; reaching peak values of 0.4 and 0.7 .

For many CubeSat constellation developers these oscillations and subsequently inconsistent coverage is acceptable. For large and sophisticated constellations, many developers require consistent ground coverage which is not possible with a drifting constellation. In order to stop this drift, each cube must perform a burn or otherwise changes its orbital velocity such that each of the cubes have identical orbital period. By matching orbital periods the cubes will no longer drift with respect to one another.

In the case of the angle-based semi-circle deployment, at the point of initial full deployment, each cube has an orbital period shown in Table 13 below. This orbital period is calculated as: ${ }^{[9]}$

$$
P=2 \pi \sqrt{\frac{a^{3}}{\mu}}
$$

where $a$ is semi major axis and $\mu$ is Earth's gravitational parameter of $398600.441 \mathrm{~km}^{3} / \mathrm{sec}^{2}$. It is advantageous for each cube to match orbital periods with the average orbital period, rather than a specific cube. While this requires every cube to be able to perform the orbital period change, it lowers the maximum $\Delta \mathrm{V}$ required to perform the maneuver. 
Table 13: Angle based separation orbital period at initial full deployment

\begin{tabular}{|c|c|c|c|c|c|}
\hline \multirow[b]{2}{*}{ Cube \# } & \multirow[b]{2}{*}{ Eccentricity } & \multirow[b]{2}{*}{$\begin{array}{c}\text { Semi-major axis } \\
(\mathbf{k m})\end{array}$} & \multicolumn{2}{|c|}{ Orbital Period } & \multirow[b]{2}{*}{$\begin{array}{l}\Delta \mathrm{T} \text { from Average } \\
(\mathrm{sec})\end{array}$} \\
\hline & & & (hour) & $\begin{array}{c}1 \times 10^{3} \\
(\mathrm{sec})\end{array}$ & \\
\hline 1 & 0.003712 & 6893.7765 & 1.5823 & 5.6964 & 0.1694 \\
\hline 2 & 0.004099 & 6896.3885 & 1.5832 & 5.6996 & 3.4072 \\
\hline 3 & 0.004290 & 6900.4580 & 1.5846 & 5.7046 & 8.4528 \\
\hline 4 & 0.003496 & 6891.0634 & 1.5814 & 5.6930 & -3.1930 \\
\hline 5 & 0.003927 & 6888.9494 & 1.5807 & 5.6904 & -5.8125 \\
\hline 6 & 0.003152 & 6896.8171 & 1.5834 & 5.7001 & 3.9385 \\
\hline 7 & 0.003921 & 6892.4186 & 1.5819 & 5.6947 & -1.5136 \\
\hline 8 & 0.003887 & 6889.2429 & 1.5808 & 5.6907 & -5.4489 \\
\hline Average & 0.0038105 & 6893.6393 & 1.5823 & 5.6962 & - \\
\hline
\end{tabular}

Changing the orbital period requires a maneuver that will change the semi-major axis of the orbit and can be performed in a multitude of ways. To provide a baseline $\Delta \mathrm{V}$ required, the provided $\Delta \mathrm{V}$ will be based on performing half of a Hohmann transfer. While this will change the eccentricity of each orbit slightly, the periods will become identical as it is independent of eccentricity.

The solution for $\Delta \mathrm{V}$ requires solving for the orbital angular momentum and orbital velocity at apogee for the pre and post maneuver orbit and follows as:

$$
\begin{gathered}
h=\sqrt{a \mu\left(1-e^{2}\right)} \\
r_{a}=\frac{h^{2}}{\mu}\left(\frac{1}{1-e}\right) \\
v=\frac{h}{r_{a}}
\end{gathered}
$$

where $h$ is the angular momentum and $r_{a}$ is the orbit radius at apogee. Solving for each cube's velocity using equations ( 14 ) through ( 16 ) and comparing its velocity against the required velocity yields the required $\Delta \mathrm{V}$. The result of this calculation is shown in Table 14 below. 
Table 14: $\Delta \mathrm{V}$ for each cube in angle based semi-circle separation

\begin{tabular}{|c|c|c|}
\hline Cube \# & $\begin{array}{c}\text { Pre-maneuver Orbital } \\
\text { Velocity }(\mathbf{k m} / \mathbf{s})\end{array}$ & $|\boldsymbol{\Delta} \mathbf{V}|$ required $(\mathbf{m} / \mathbf{s})$ \\
\hline Target Orbit & 7.5751 & - \\
\hline 1 & 7.5758 & 0.6708 \\
\hline 2 & 7.5714 & 3.6947 \\
\hline 3 & 7.5678 & 7.3733 \\
\hline 4 & 7.5789 & 3.7989 \\
\hline 5 & 7.5768 & 1.6953 \\
\hline 6 & 7.5784 & 3.2434 \\
\hline 7 & 7.5750 & 0.1663 \\
\hline 8 & 7.5770 & 1.8370 \\
\hline
\end{tabular}

Based on these calculations the average $\Delta \mathrm{V}$ is $2.81 \mathrm{~m} / \mathrm{s}$ while the maximum for this case is 7.37 $\mathrm{m} / \mathrm{s}$. Even with a design margin of 2:1 each CubeSat would only be required to induce approximately $15 \mathrm{~m} / \mathrm{s}$ of $\Delta \mathrm{V}$.

Multiple propulsion system, both currently available and under development, provide well over $15 \mathrm{~m} / \mathrm{s}$ of $\Delta \mathrm{V} ;{ }^{[16]}$ allowing a CubeSat developer to easily stop the breakdown of a developed constellation. As discussed in section 1.6.2 this $\Delta \mathrm{V}$ could also be imparted using variable drag control.

\subsection{Specific Launch Sites}

Launching a constellation from different launch sites will produce different coverage quality of the desired ground region. In order to understand this effect four common launch inclinations corresponding to major launch sites are analyzed. Each orbit from this inclination is designed as a 500 x $550 \mathrm{~km}$ orbit with the launch site dictating the inclination. Each launch site will have a set of 8 cubes separated using the Y-vector based separation discussed in section 4.1.2. The constellations are only analyzed around their full deployment under the assumption that the constellation breakdown will be mitigated with an orbital maneuver. The coverage metrics are then determined based on a weeklong interval centered on the day of initial full deployment.

It is important to keep track of the satellite ground track over the one week timespan. Every period the ground track shifts an equal longitudinal distance described by ${ }^{[17]}$ : 


$$
\Delta \alpha_{\text {orbit }}=\omega_{\mathrm{R}} P
$$

where $\mathrm{P}$ is the orbital period of the satellite and $\omega_{R}$ is angular velocity of the Earth relative to the orbital plane. After accounting for the effect of eccentricity $\omega_{R}$ is expressed as ${ }^{[17]}$ :

$$
\omega_{R} \approx-0.004178075-2.396288 \times 10^{9} \mathrm{a}^{-(7 / 2)}\left(1-e^{2}\right)^{-2} \cos (i)
$$

where $\omega_{R}$ is in degrees / period, $e$ is the orbital eccentricity, $a$ is the orbital semi-major axis in kilometers, and $i$ is the orbital inclination. This equation is based on an approximation of the Earth's Oblateness.

Given the $500 \times 550 \mathrm{~km}$ altitude of the orbit, the eccentricity of the orbit is 0.00362154 and a semi-major axis of 6903.14 kilometers. Using the period of the orbit of 95.133 minutes, found using equation ( 13 ), the ground track shift is calculated and shown in Table 15. Based on the ground track repetition rate each Cube will return over a specific ground location approximately every day. Over one week this results in approximately seven full ground track shifts over the given regions.

Table 15: Specific launch site ground track times

\begin{tabular}{|c|c|c|c|c|}
\hline Launch Site & Inclination (deg) & $\begin{array}{c}\text { Ground track } \\
\text { shift (deg/Period) }\end{array}$ & $\begin{array}{c}\text { Full Shift (\# of } \\
\text { orbital Periods) }\end{array}$ & $\begin{array}{c}\text { Full Shift } \\
\text { (days) }\end{array}$ \\
\hline CCAFS & 51.6 & 24.16 & 14.90 & .984 \\
\hline CCAFS & 28.5 & 24.29 & 14.82 & .979 \\
\hline VAFB & $98\left(180^{\circ}\right.$ RAAN $)$ & 23.78 & 15.14 & 1.00 \\
\hline Wallops & 45.0 & 24.23 & 14.86 & .982 \\
\hline
\end{tabular}

The results of each coverage analysis are provided in the following sections. Each launch site has

the minimum, maximum, average, standard deviation, total sum, and the total region coverage percentage provided for the coverage and revisit time metrics. The total sum metric is calculated by summing all of the grid zones in the region and maxes out at 260,519 days by assuming each grid zone has a maximum value of 1 week. 
For cases in which full coverage is not established, the grid points outside of the coverage area will result in zero seconds for coverage time and 1 week (604800 seconds) for revisit time. The reported metrics for these cases do not include grid points that are not covered as including them can skew the metrics. By include grid points that are not covered the revisit times are extremely skewed as each non-covered grid point maxes out to a value of 1 week. Each grid point that is not covered in the region adds 7 days to the revisit time sum metric and with an extremely large amount of points the sum metric would increase by orders of magnitude. This has the side-effect of increasing the average coverage time for a region with partial coverage. When examining only the average time this can misdirect any formed conclusions from the data, however, in conjunction with the sum metric any conclusions on the overall coverage and revisit time are still valid.

\subsubsection{CCAFS $-\mathbf{5 1 . 6}^{\circ}$}

From the Cape Canaveral Air Force Station a $51.6^{\circ}$ inclination orbit is often launched as this is the International Space Stations inclination. The results of the coverage metrics are shown in Table 16 below with contour plots for each case and metric shown in Figure 29 and Figure 30 below. The contour plots graphically depict the coverage the constellation provides on a color scale from blue to red with color legends provided to the right. In the coverage time contours a color of red is considered a better result, while a color of blue is considered better in the revisit time contour.

Table 16: CCAFS - 51.6 degree inclination coverage metrics

\begin{tabular}{|l|c|c|c|c|}
\hline \multirow{2}{*}{$\begin{array}{c}\text { Metric Values } \\
(\mathbf{m i n})\end{array}$} & \multicolumn{2}{|c|}{ Case 1 } & \multicolumn{2}{c|}{ Case 2 } \\
\cline { 2 - 5 } & Coverage Time & Revisit Time & Coverage Time & Revisit Time \\
\hline Min & 43.703 & 62.738 & 28.200 & 196.447 \\
\hline Max & 181.371 & 208.845 & 61.196 & 418.825 \\
\hline Average & 105.355 & 120.289 & 41.346 & 287.733 \\
\hline Std. Deviation & 37.165 & 35.547 & 6.586 & 38.460 \\
\hline Sum (days) & 177.786 & 202.987 & 1068.597 & 7436.500 \\
\hline \% Coverage & \multicolumn{2}{|c|}{100} & \multicolumn{2}{c|}{100} \\
\hline
\end{tabular}




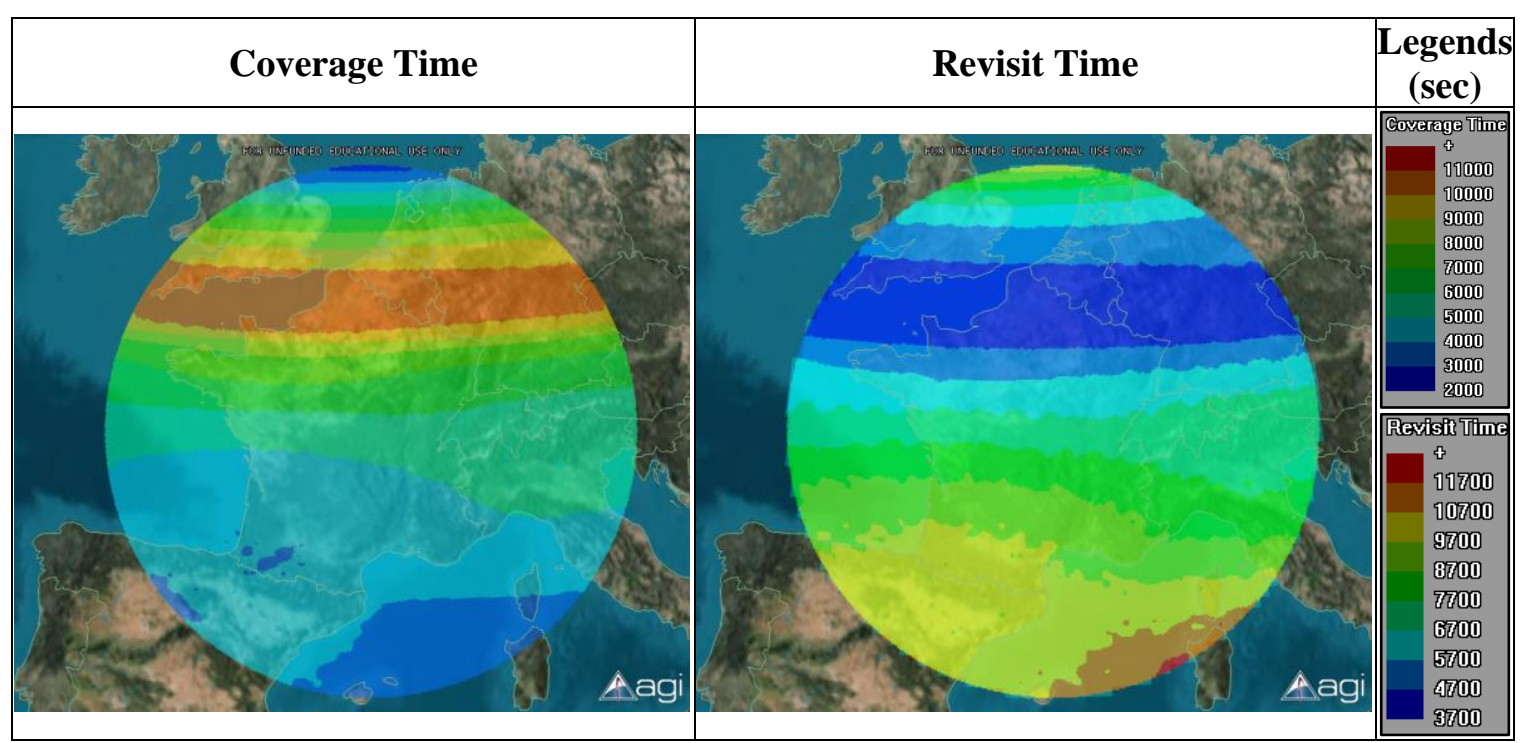

Figure 29: Case 1 coverage contour from CCAFS - 51.6 ${ }^{\circ}$ orbit

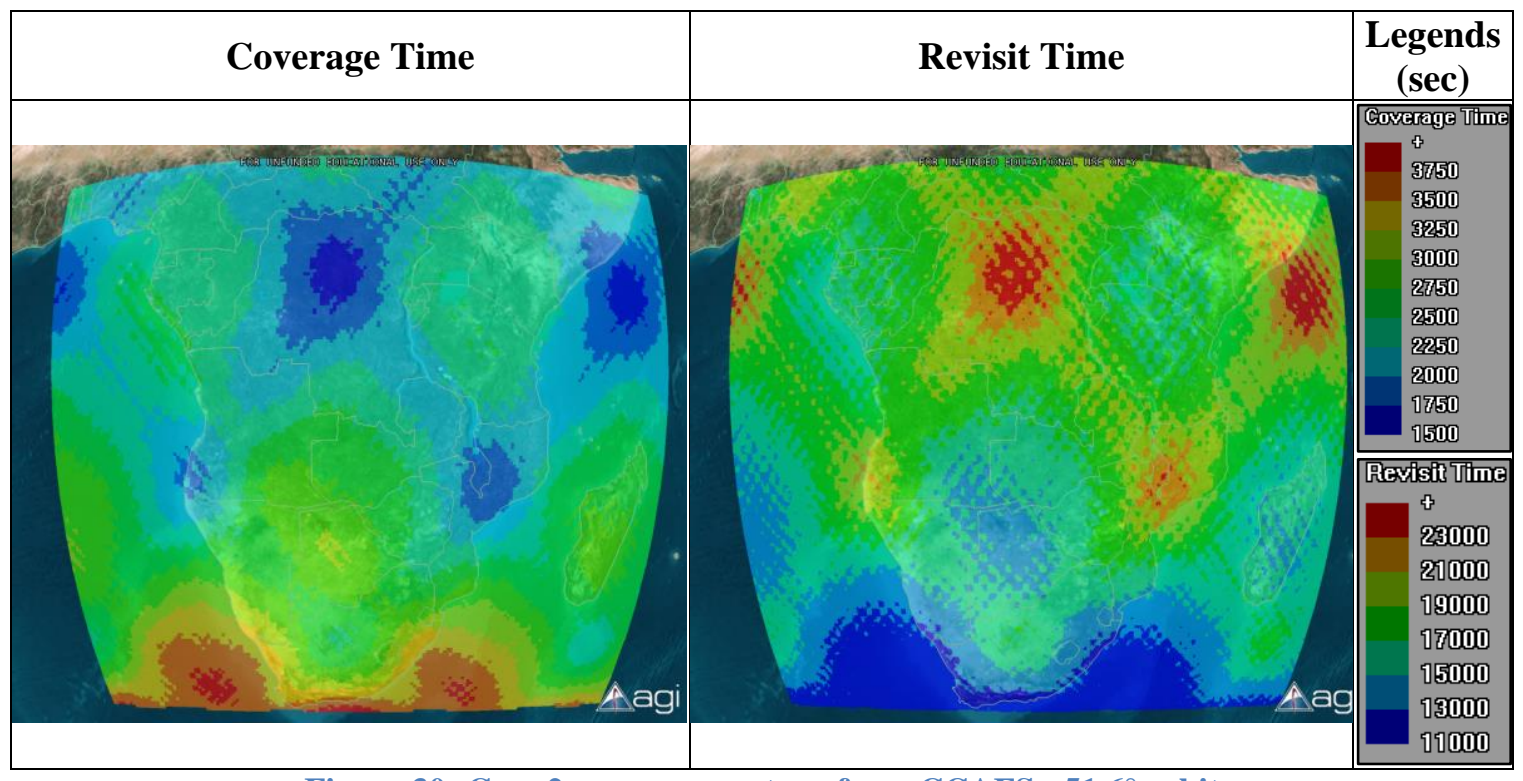

Figure 30: Case 2 coverage contour from CCAFS $-51.6^{\circ}$ orbit

\subsubsection{CCAFS $-28.5^{\circ}$}

Cape Canaveral Air Force station also launches into GEO regularly which requires launching at the lowest inclination available to the launch site; for CCAFS this translates to a $28.5^{\circ}$ inclination. From this inclination, the constellation metrics are shown in Table 17 below, with contour plots for Case 2 metric shown in Figure 32. Note that due to the orbital inclination all of the Case 1 and part of Case 2 are not covered. As shown in Figure 31 below the southernmost point on the 2D projection that the satellites obtain does not fully cover the Case 2 region. Further; the 
northernmost point of the satellites orbits never crosses into the Case 1 region; thus never providing coverage.

Table 17: CCAFS $-28.5^{\circ}$ inclination coverage metrics

\begin{tabular}{|c|c|c|c|c|}
\hline \multirow{2}{*}{$\begin{array}{l}\text { Metric Values } \\
\quad(\text { min) }\end{array}$} & \multicolumn{2}{|c|}{ Case 1} & \multicolumn{2}{|c|}{ Case 2} \\
\hline & Coverage Time & Revisit Time & Coverage Time & Revisit Time \\
\hline Min & \multirow{6}{*}{\multicolumn{2}{|c|}{ NO COVERAGE }} & 0.400 & 57.142 \\
\hline Max & & & 213.191 & 2519.900 \\
\hline Average & & & 82.683 & 168.667 \\
\hline Std. Deviation & & & 38.876 & 117.610 \\
\hline Sum (days) & & & 1990.236 & 4059.966 \\
\hline$\%$ Coverage & & & \multicolumn{2}{|c|}{93.00} \\
\hline
\end{tabular}

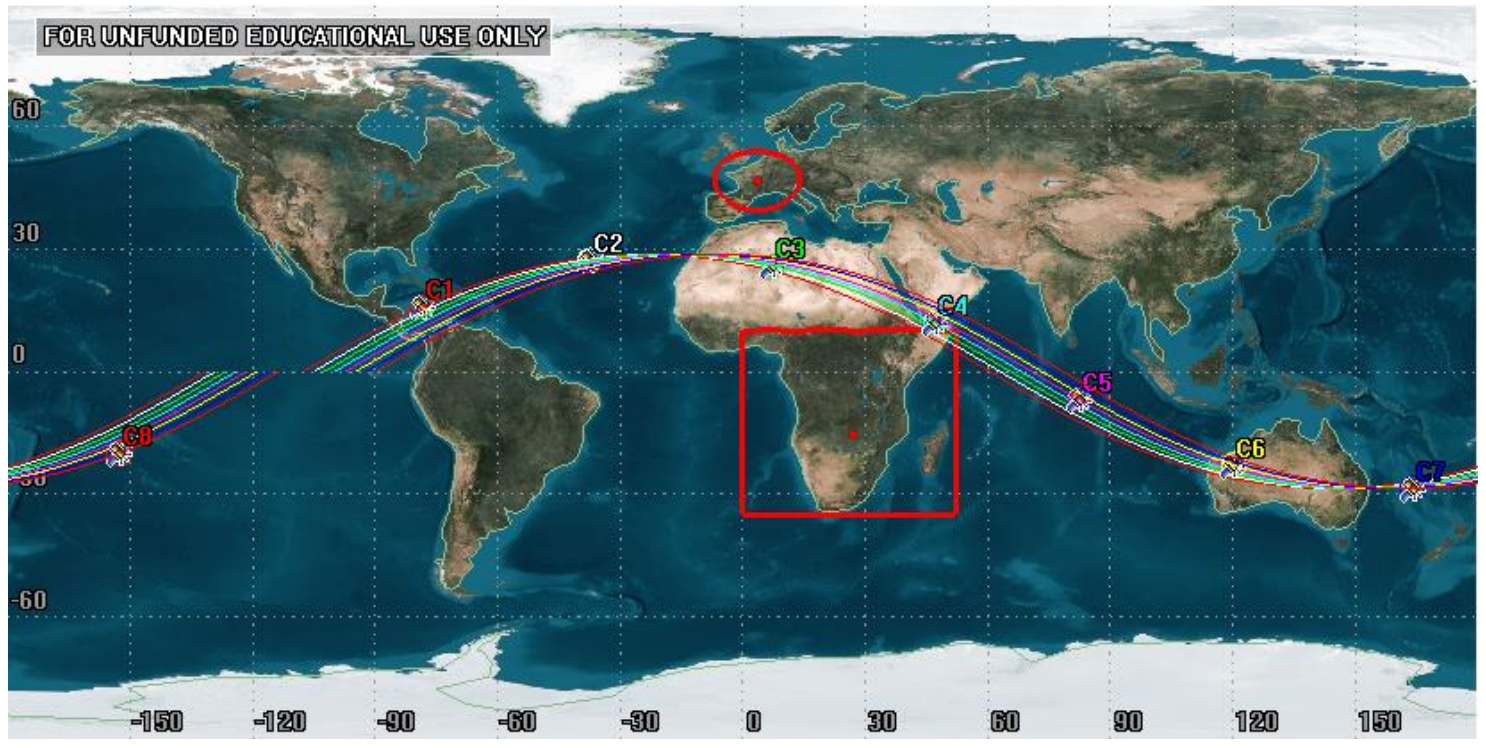

Figure 31: 2D projection of $28.5^{\circ}$ orbit and relation to Case 1 and 2 


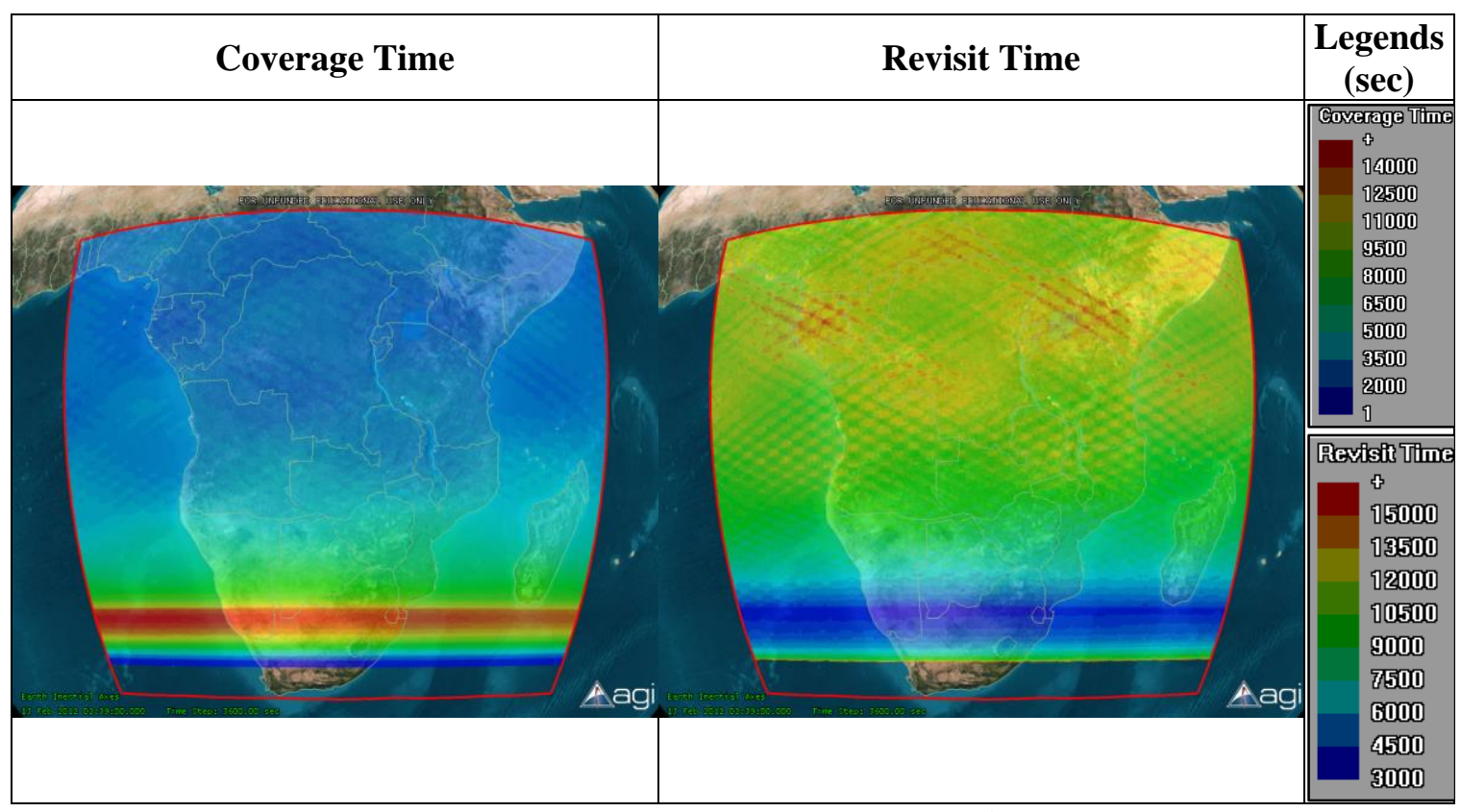

Figure 32: Case 2 coverage contour from CCAFS $-28.5^{\circ}$ orbit

\subsubsection{VAFB $-98^{\circ}$ with $180^{\circ}$ RAAN}

Vandenberg Air Force Base regularly launches polar and sun-synchronous satellites with orbits near retrograde $98^{\circ}$ of inclination. This is expressed as a positive inclination of $98^{\circ}$ with a RAAN value of $180^{\circ}$ as VAFB must launch in a southern direction. The resulting constellation metrics and contour plots are shown in Table 18, Figure 33, and Figure 34 below.

Table 18: VAFB $-98^{\circ}$ retro inclination coverage metrics

\begin{tabular}{|l|c|c|c|c|}
\hline \multirow{2}{*}{$\begin{array}{c}\text { Metric Values } \\
(\text { min) }\end{array}$} & \multicolumn{2}{|c|}{ Case 1 } & \multicolumn{2}{c|}{ Case 2 } \\
\cline { 2 - 5 } & Coverage Time & Revisit Time & Coverage Time & Revisit Time \\
\hline Min & 36.458 & 208.931 & 25.706 & 271.333 \\
\hline Max & 51.302 & 304.346 & 40.785 & 457.013 \\
\hline Average & 43.023 & 253.676 & 32.045 & 347.871 \\
\hline Std. Deviation & 3.161 & 18.900 & 2.939 & 27.829 \\
\hline Sum (days) & 72.601 & 428.079 & 828.217 & 8990.765 \\
\hline$\%$ Coverage & \multicolumn{2}{|c|}{100} & \multicolumn{2}{c|}{100} \\
\hline
\end{tabular}




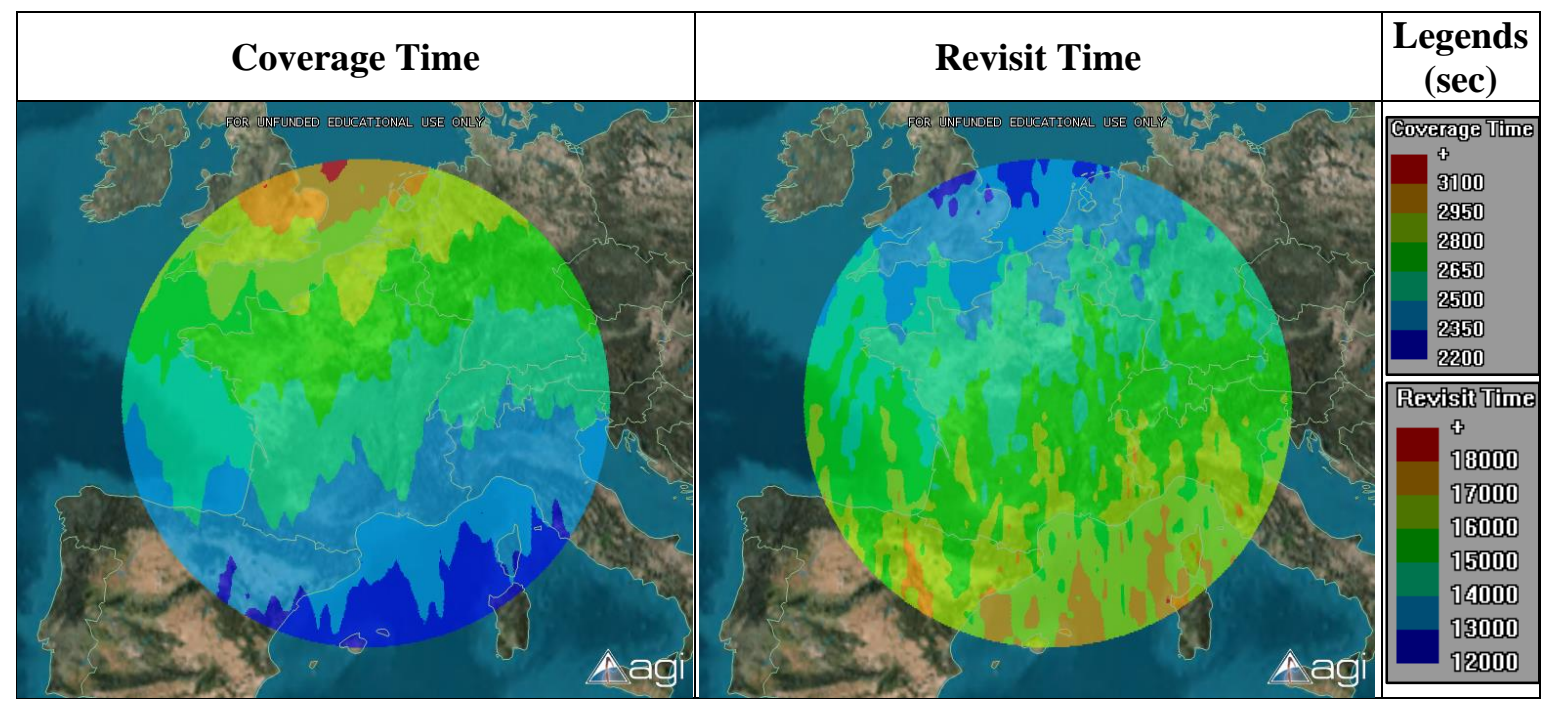

Figure 33: Case 1 coverage contours from VAFB - $98^{\circ}$ retro orbit

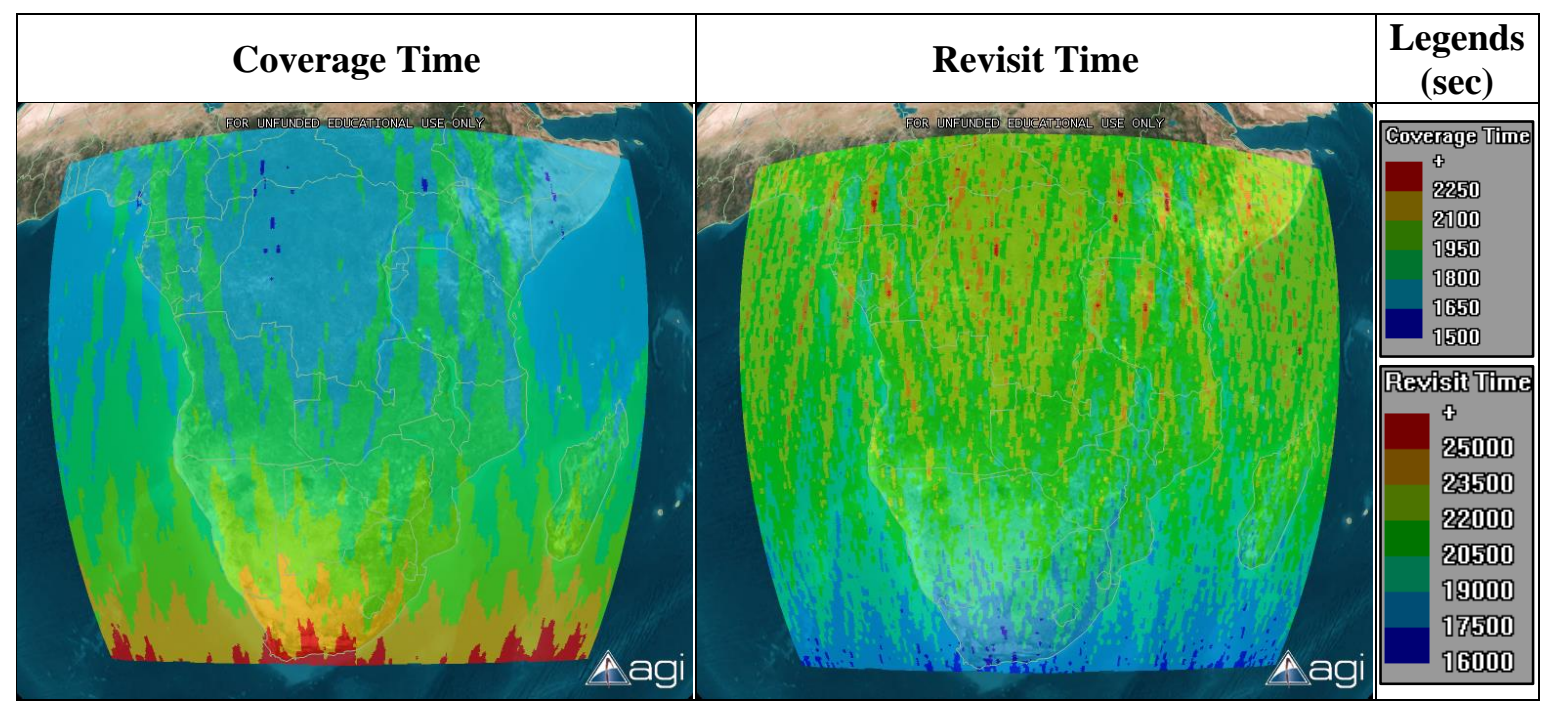

Figure 34 Case 1 coverage contours from VAFB - $98^{\circ}$ retro orbit

\subsubsection{WALLOPS $-\mathbf{4 5}^{\circ}$}

The NASA Wallops Flight Facility is capable of launching into inclinations between 38 and 60 degrees. An inclination of 45 degrees was selected for analysis as it partially covers Case 1 and fully covers Case 2. The results of the Wallops launch site analysis are found in Table 19, Figure 35, and Figure 36. 
Table 19: Wallops $-45^{\circ}$ retro inclination coverage metrics

\begin{tabular}{|l|c|c|c|c|}
\hline \multirow{2}{*}{$\begin{array}{c}\text { Metric Values } \\
(\mathbf{m i n})\end{array}$} & \multicolumn{2}{|c|}{ Case 1 } & \multicolumn{2}{c|}{ Case 2 } \\
\cline { 2 - 5 } & Coverage Time & Revisit Time & Coverage Time & Revisit Time \\
\hline Min & 6.606 & 62.231 & 33.004 & 151.591 \\
\hline Max & 197.661 & 530.178 & 75.142 & 372.111 \\
\hline Average & 131.949 & 117.132 & 45.438 & 259.629 \\
\hline Std. Deviation & 51.690 & 73.765 & 7.858 & 38.536 \\
\hline Sum (days) & 140.654 & 119.083 & 1174.340 & 6710.156 \\
\hline \% Coverage & \multicolumn{2}{|c|}{63.84} & \multicolumn{2}{c|}{100} \\
\hline
\end{tabular}

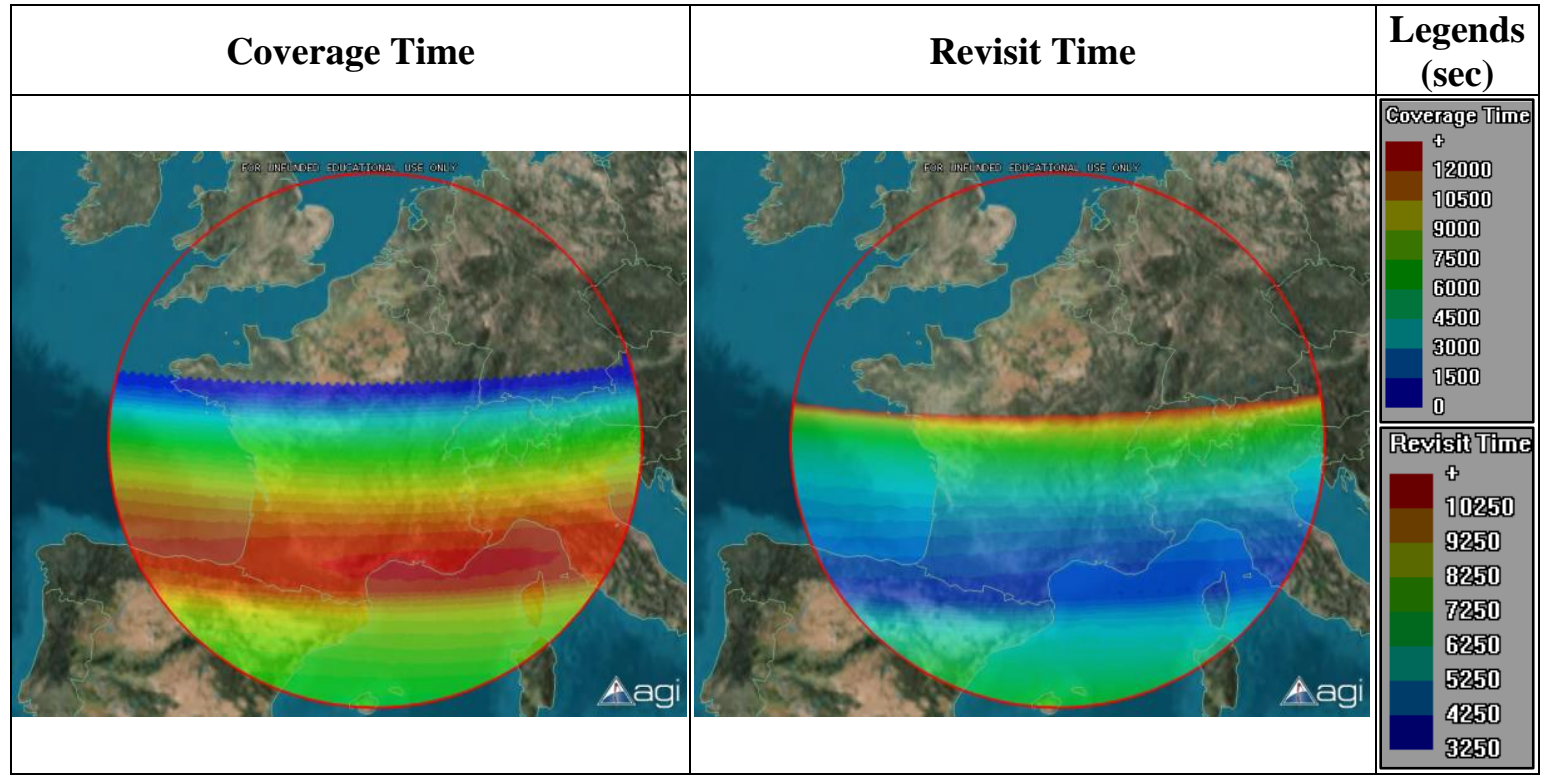

Figure 35: Case 1 coverage contours from Wallops - $45^{\circ}$ orbit

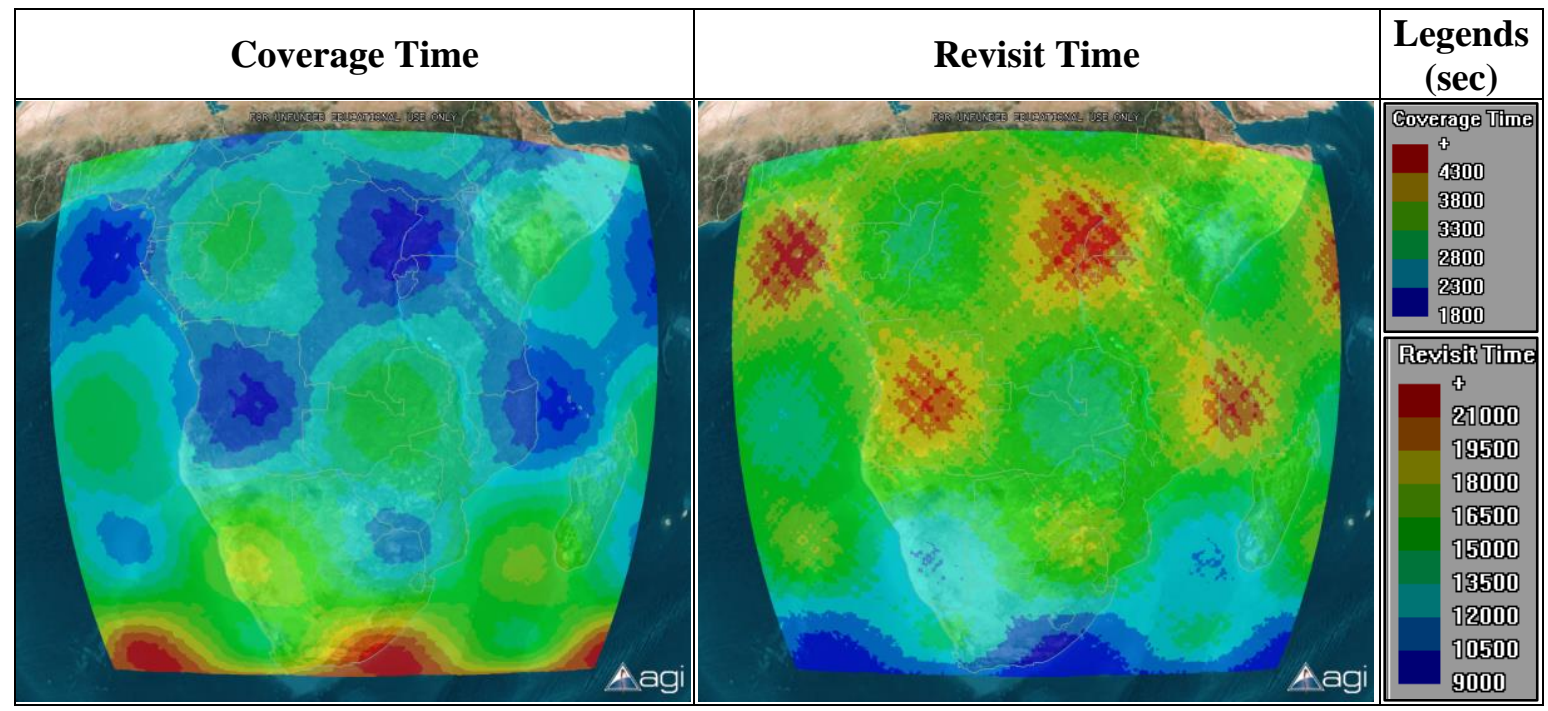

Figure 36: Case 2 coverage contours from Wallops - $45^{\circ}$ orbit 


\subsubsection{Comparison of Specific Launch Sites}

To understand the implications of each launch site based coverage they are compared by each Case, since the results of Case 1 cannot be used to compare against Case 2 .

\subsubsection{Case 1}

The coverage results for Case 1 are mixed as only two of the four launch sites provide full coverage of the constellation. The CCAFS $-28.5^{\circ}$ and Wallops launches provided 0 and $63.84 \%$ coverage respectively. The coverage time metric is shown in Table 20 below and has varied results.

The Wallops launch has the highest average coverage time with approximately 130 minutes of coverage time per grid point while only covering $63 \%$ of the region. The next highest average is found in the CCAFS- $51.6^{\circ}$ launch with a difference in average coverage time of approximately 30 minutes; however, this launch covers $100 \%$ of the region. While both Wallops and CCAFS $51.6^{\circ}$ have similar average values the Wallops launch has a higher standard deviation and lower sum. This is due to the fact that the Wallops launch only partially covers the region. The grid points adjacent to the non-covered grid points have extremely low coverage times (manifested as the 3.6 minute minimum) leading to a lower average and larger standard deviation. For CubeSat developers interested in the entire region the $45^{\circ}$ inclination Wallops launch would not be sufficient. If only a portion of the region is of interest the lower inclination provides, on average, higher coverage times. This trend holds true when examining the VAFB launch at $98^{\circ}$ which has a significantly lower average and sum. Importantly though, the coverage provided by Vandenberg is significantly more consistent with a smaller maximum to minimum range and significantly lower standard deviation.

While correlated to the coverage time metric the revisit time metric, shown in Table 21, provides valuable insight into the performance of each launch. In the revisit time metric the Vandenberg 
AFB launch has the highest average; given the low average coverage time this is the expected result. As the cubes in the constellation do not spend much time over the region, their associated revisit time will be higher. Consistently, the Wallops launch has the lowest revisit time and highest coverage time averages.

For the CCAF - 51.6 launch the revisit time metric is close to that of the Wallops launch with a different of only approximately 6 minutes. While the averages are the same the maximum to minimum range is much smaller than that of Wallops, resulting in a lower standard deviation. It is important to note the sum metric for the CCAFS $-51.6^{\circ}$ is significantly higher than that of the Wallops launch and is due to the method used to calculate the Wallops statistical metrics, as discussed in section 4.3. However, the CCAFS $-51.6^{\circ}$ launch provides a more consistent revisit time metric than the Wallops launch at $45^{\circ}$ of inclination.

The VAFB launch provides the most consistent revisit time with a standard deviation of approximately 20 minutes and a max-min range of 100 minutes, but with the highest average revisit time. This standard deviation is nearly half of that of CCAFS $-51.6^{\circ}$ and nearly 5 times less than the Wallops launch. As with coverage time this low standard deviation is linked to low overall coverage; yielding a significantly higher sum that is twice that of CCAFS - $51.6^{\circ}$ and four times that of Wallops. The cubes in the constellation launched from VAFB fully cover the Case 1 region but also cover areas of higher latitude. By covering areas of higher latitudes than required the constellation coverage results in a longer revisit time and a shorter coverage time.

Case 1, being at relatively high latitude requires a constellation of a sufficient inclination to provide full or even partial coverage. A higher constellation inclination results in decreased coverage times and increased revisit times, but also increased consistency across the region. The ideal single plane solution for Case 1 is a constellation with an inclination that fully covers the Case 1 region but is not too high. The satellites in this constellation would not spend a significant 
amount of time in higher latitudes and thus providing higher coverage times, lower revisit times, and a lower standard deviation.

Table 20: Case 1 coverage time metric summary

\begin{tabular}{|c|c|c|c|c|c|c|}
\hline Launch & $\begin{array}{c}\text { Min } \\
(\mathbf{m i n})\end{array}$ & $\begin{array}{c}\text { Max } \\
(\mathbf{m i n})\end{array}$ & $\begin{array}{c}\text { Ave } \\
(\mathbf{m i n})\end{array}$ & $\begin{array}{c}\text { Std. Dev. } \\
(\mathbf{m i n})\end{array}$ & $\begin{array}{c}\text { Sum } \\
(\mathbf{d a y})\end{array}$ & $\%$ Coverage \\
\hline CCAFS -51.6 & 43.703 & 181.371 & 105.355 & 37.165 & 177.786 & 100 \\
\hline $\begin{array}{c}\text { CCAFS - } \\
28.5^{\circ}\end{array}$ & \multicolumn{7}{|c|}{ No Coverage } & 0 \\
\hline VAFB - 98 & 36.458 & 51.302 & 43.023 & 3.161 & 72.601 & 100 \\
\hline Wallops $45^{\circ}$ & 6.606 & 197.661 & 131.949 & 51.69 & 140.654 & 63.84 \\
\hline
\end{tabular}

Table 21: Case 1 revisit time metric summary

\begin{tabular}{|c|c|c|c|c|c|c|}
\hline Launch & $\begin{array}{c}\text { Min } \\
(\mathbf{m i n})\end{array}$ & $\begin{array}{c}\text { Max } \\
(\mathbf{m i n})\end{array}$ & $\begin{array}{c}\text { Ave } \\
(\mathbf{m i n})\end{array}$ & $\begin{array}{c}\text { Std. Dev. } \\
(\mathbf{m i n})\end{array}$ & $\begin{array}{c}\text { Sum } \\
(\mathbf{d a y})\end{array}$ & $\begin{array}{c}\% \\
\text { Coverage }\end{array}$ \\
\hline CCAFS -51.6 & 62.738 & 208.845 & 120.289 & 35.547 & 202.987 & 100 \\
\hline $\begin{array}{c}\text { CCAFS - } \\
28.5^{\circ}\end{array}$ & \multicolumn{7}{|c|}{ No Coverage } & 0 \\
\hline VAFB - 98 & 208.931 & 304.346 & 253.676 & 18.9 & 428.079 & 100 \\
\hline Wallops 45 & 62.231 & 530.178 & 117.132 & 73.765 & 119.083 & 63.84 \\
\hline
\end{tabular}

\subsubsection{Case 2}

For the Case 2 region, the CCAFS $-28.5^{\circ}$ launch provides $93 \%$ coverage while the other launches provide $100 \%$ coverage. The results of the Case 2 analysis are similar to the trends found in the Case 1 analysis however they are less dramatic as the latitude of the Case 2 region is closer to the equator.

While only providing $93 \%$ coverage the CCAFS $-28.5^{\circ}$ launch has the highest average coverage time of 84 minutes; almost twice that of the next highest coverage time average of 44 minutes. However, due to not providing full coverage the standard deviation of the launch's coverage time is significantly higher than the other launches. This effect leads to a coverage time that is highly varied with large peaks, as shown in Figure 32. The CCAFS $-28.5^{\circ}$ launch results in a selective band of high coverage time that does not extend into the majority of the region. 
The results from the CCAFS $-51.6^{\circ}$ and the Wallops $-45^{\circ}$ launches are extremely close to one another in all of the coverage time metrics; however, in the revisit time metrics the Wallops launch is slightly better. With an average revisit time approximately 20 minutes less than that of the CCAFS $-51.6^{\circ}$ launch, the Wallops launch is slightly more optimal for a ground user.

As in the Case 1 analysis the VAFB launch at $98^{\circ}$ provides the lowest average coverage time and highest average revisit time, however, it also provides the most consistent coverage time and revisit time metrics. The VAFB launch is significantly more consistent when examining the range of the maximum to minimum and the standard deviation. Consistency is a useful trait for a ground user in a significantly large area such as Case 2 as the same coverage can be expected anywhere in the region.

Table 22: Case 2 coverage time metric summary

\begin{tabular}{|l|c|c|c|c|c|c|}
\hline \multicolumn{1}{|c|}{ Launch } & $\begin{array}{c}\text { Min } \\
(\mathbf{m i n})\end{array}$ & $\begin{array}{c}\text { Max } \\
(\mathbf{m i n})\end{array}$ & $\begin{array}{c}\text { Ave } \\
(\mathbf{m i n})\end{array}$ & $\begin{array}{c}\text { Std. Dev. } \\
(\mathbf{m i n})\end{array}$ & $\begin{array}{c}\text { Sum } \\
(\mathbf{d a y})\end{array}$ & $\begin{array}{c}\% \\
\text { Coverage }\end{array}$ \\
\hline $\begin{array}{l}\text { CCAFS - } \\
51.6^{\circ}\end{array}$ & 28.200 & 61.196 & 41.346 & 6.586 & 1068.597 & 100 \\
\hline $\begin{array}{l}\text { CCAFS - } \\
28.5^{\circ}\end{array}$ & 0.4 & 213.191 & 82.683 & 38.876 & 1990.236 & 93 \\
\hline VAFB $-98^{\circ}$ & 25.706 & 40.785 & 32.045 & 2.939 & 828.217 & 100 \\
\hline Wallops $45^{\circ}$ & 33.004 & 75.142 & 45.438 & 7.858 & 1174.34 & 100 \\
\hline
\end{tabular}

Table 23: Case 2 revisit time metric summary

\begin{tabular}{|l|c|c|c|c|c|c|}
\hline \multicolumn{1}{|c|}{ Launch } & $\begin{array}{c}\text { Min } \\
(\mathbf{m i n})\end{array}$ & $\begin{array}{c}\text { Max } \\
(\mathbf{m i n})\end{array}$ & $\begin{array}{c}\text { Ave } \\
(\mathbf{m i n})\end{array}$ & $\begin{array}{c}\text { Std. Dev. } \\
(\mathbf{m i n})\end{array}$ & $\begin{array}{c}\text { Sum } \\
(\mathbf{d a y})\end{array}$ & $\begin{array}{c}\% \\
\text { Coverage }\end{array}$ \\
\hline $\begin{array}{l}\text { CCAFS - } \\
51.6^{\circ}\end{array}$ & 196.447 & 418.825 & 287.733 & 38.46 & 7436.5 & 100 \\
\hline $\begin{array}{l}\text { CCAFS - } \\
28.5^{\circ}\end{array}$ & 57.142 & 2519.9 & 168.667 & 117.61 & 4059.966 & 93 \\
\hline VAFB $-98^{\circ}$ & 271.333 & 457.013 & 347.871 & 27.829 & 8990.765 & 100 \\
\hline Wallops $45^{\circ}$ & 151.591 & 372.111 & 259.629 & 38.536 & 6710.156 & 100 \\
\hline
\end{tabular}

\subsection{Multi-plane Analysis}

When examining the data in section 4.3 it becomes clear that both CCAFS launches and the

Wallops launch are similar; especially when compared to the results of VAFB. These three launches provide a dramatically different coverage type than that provided by Vandenberg's high 
inclination. While providing relatively low metrics the VAFB launch does provide very consistent values. Many developers may want both high coverage and consistent values; leading them to attempt to augment a standard prograde orbit with that of a high inclination retrograde orbit such as the VAFB launch. Such a launch scenario would likely include the VAFB and CCAFS $-51.6^{\circ}$ launches. The CCAFS $-28.5^{\circ}$ launch does not provide any coverage to Case 1 and only partial coverage to Case 2. Further, with the increase of launches to the ISS from CCAFS at a $51.6^{\circ}$ inclination, this launch scenario will occur much more often than a launch into a $45^{\circ}$ inclination.

In the augmented constellation, both launched inclination planes use the same 500 x $550 \mathrm{~km}$ altitude orbit at $51.6^{\circ}$ and $98^{\circ}$ inclinations previously examined. Eight Cubes are separated using the Y-vector based semi-circle separation scheme. As both inclinations have a time to full deployment between 45 and 50 days the analysis is centered at 47.5 days with a full range of 1 week. The results of the two plane analysis are shown in Table 24, Figure 38, and Figure 39 below.

Table 24: Two plane constellation coverage metrics

\begin{tabular}{|l|c|c|c|c|}
\hline \multirow{2}{*}{$\begin{array}{c}\text { Metric Values } \\
(\mathbf{m i n})\end{array}$} & \multicolumn{2}{|c|}{ Case 1 } & \multicolumn{2}{c|}{ Case 2 } \\
\cline { 2 - 5 } & Coverage Time & Revisit Time & Coverage Time & Revisit Time \\
\hline Min & 89.524 & 49.587 & 56.601 & 112.074 \\
\hline Max & 227.095 & 126.459 & 105.413 & 204.559 \\
\hline Average & 146.758 & 81.853 & 74.178 & 157.737 \\
\hline Std. Deviation & 39.606 & 18.936 & 10.127 & 17.495 \\
\hline Sum (days) & 247.654 & 138.127 & 1917.133 & 4076.729 \\
\hline \% Coverage & \multicolumn{2}{|c|}{100} & \multicolumn{2}{c|}{100} \\
\hline
\end{tabular}

The results of the analysis in Case 1 show much more consistent coverage and revisit times with an overall high average. The two plane constellation has an average coverage time of 146 minutes, higher than any of the single plane coverage times. The constellation also has a total sum of 247 days, which is the addition of the single plane sums of the CCAFS $-51.6^{\circ}$ and the VAFB launches. This is not a coincidence, as coverage time will stack as more satellites provide 
coverage to the constellation; so long as two or more satellites do not cover the same grid point. Based on the results shown in Table 24, the use of a mid and a high inclination launch results in increase in the average and sum coverage time metrics. It also provides a better standard deviation than any of the single mid-range inclination launches.

The revisit time metric for Case 1 also shows similar trends. Unlike the coverage time metric, the revisit time metric does not simply add together. The addition of a second plane, of a sufficiently different inclination, results in a largely spread out constellation. As shown in Figure 37, as one cube leaves Case 1 (Cube C4) another cube (Cube C12) quickly arrives at the region to provide coverage. This kind of spread significantly lowers the revisit time for a given region. In the case of a single plane constellation, once the Earth rotates from underneath the constellation a ground user must wait for the Earth to rotate 180 degrees before coverage is re-established. For a two plane constellation, a ground user could wait for the Earth to rotate as little as 90 degrees. 


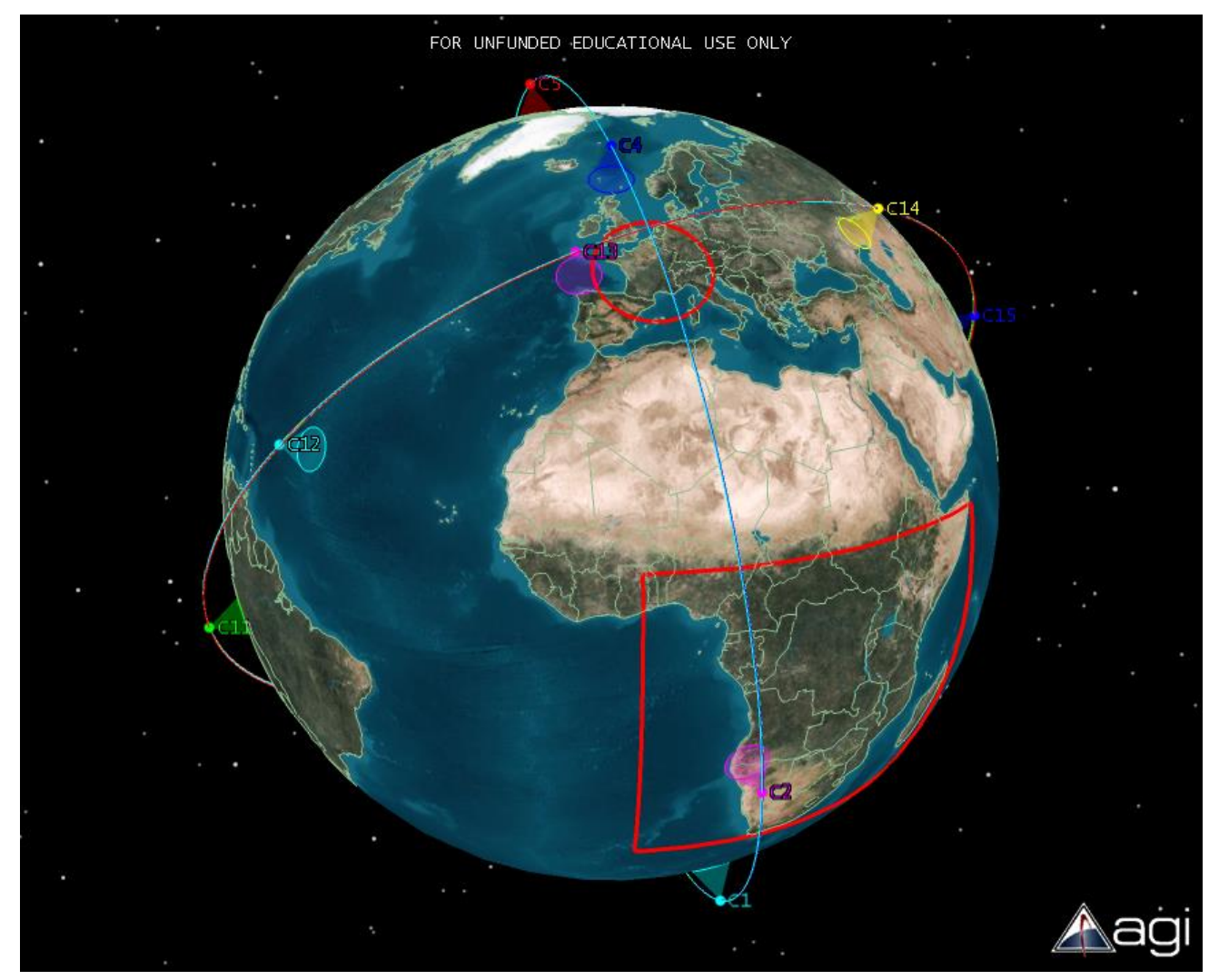

Figure 37: Two plane constellation Cube locations

This effect is why the revisit time metric for the two plane constellation is significantly better than any of the single plane results, with the lowest minimum, maximum, standard deviation, and average revisit times for Case 1. It is important to note; that this effect is heightened for Case 1. As shown in Figure 37 the two planes intersect at latitude over Case 1 during the coverage analysis interval. Further if the interval selected was further into the future the RAAN drift would case the near $90^{\circ}$ separation between the two launch planes to oscillate. This effect will still play a major factor in Case 2 but not as significantly as in Case 1. 


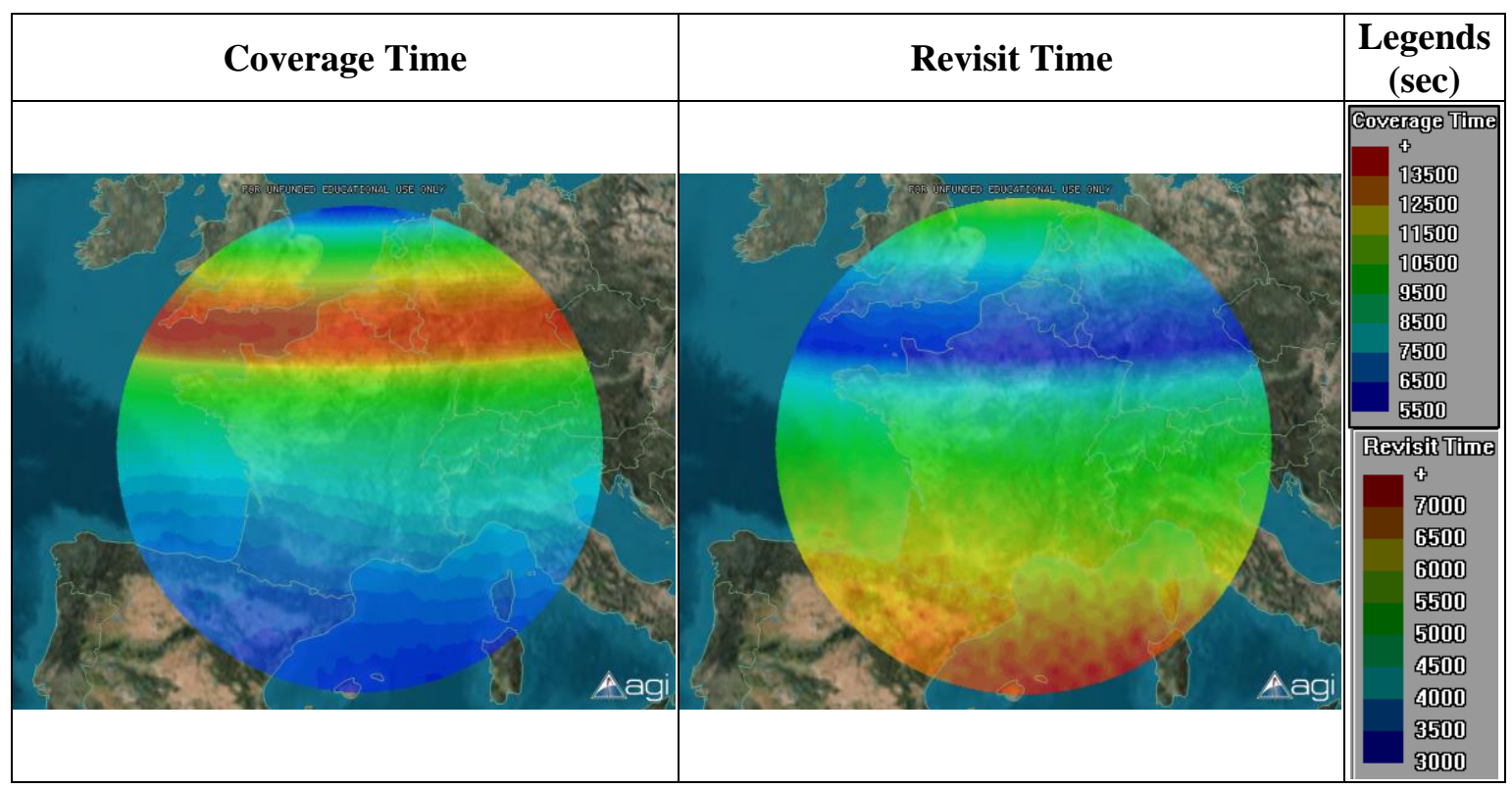

Figure 38: Case 1 coverage contours for a two plane constellation

The trends in the analysis of Case 2 are similar to the trends found in the analysis of Case 1 . An average coverage time of 72 minutes is better than two of the single plane launches and close to that of the CCAFS $-28.5^{\circ}$ launch. However, the two plane constellation has a significantly lower standard deviation than the CCAFS $-28.5^{\circ}$ launch while maintaining a close average.

The two plane constellation has the lowest average, maximum, standard deviation, and sum revisit times for Case 2. As with Case 1, the augmentation of another plane with sufficiently different inclination allows for a cube to return to the region quicker than with a single plane. 


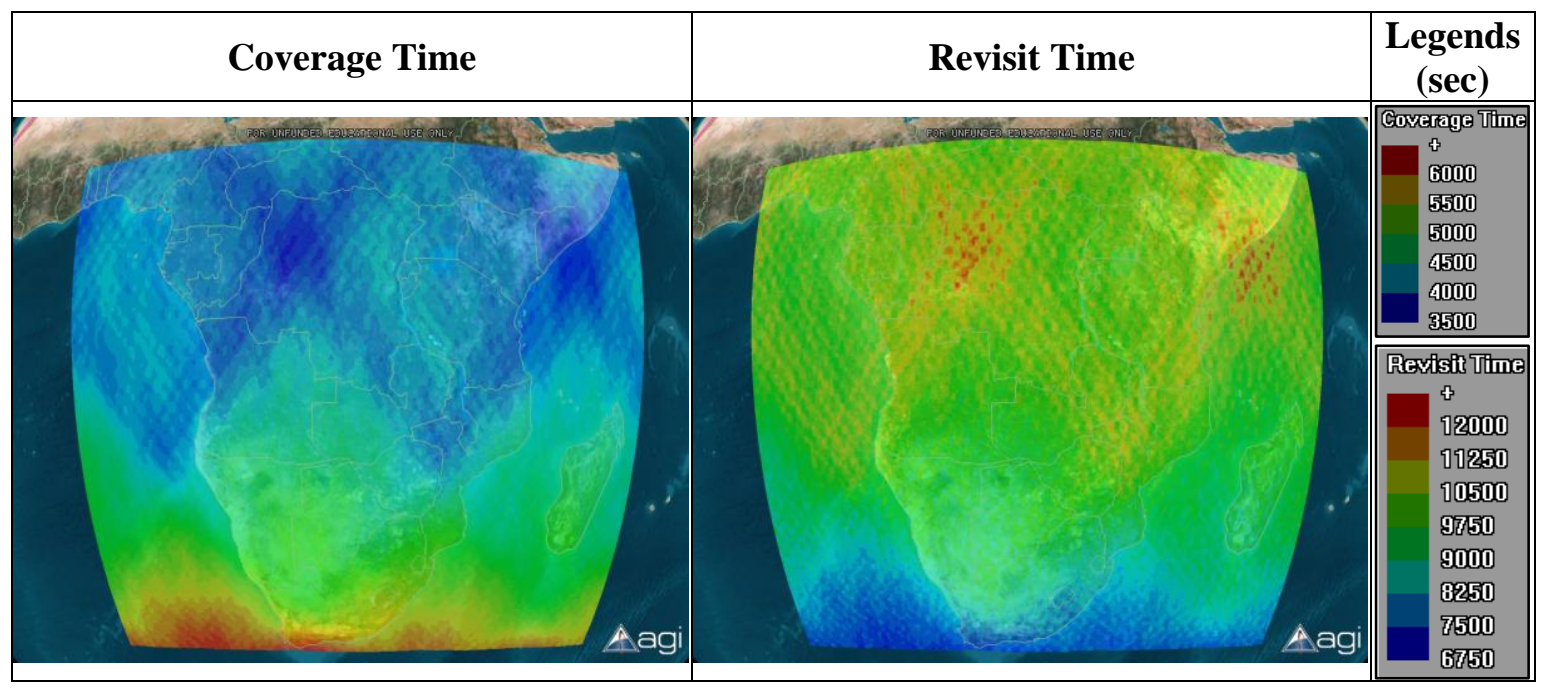

Figure 39: Case 2 coverage contours for a two plane constellation

A key detail that affects the revisit time metric is the longitudinal offset (RAAN shift) of each plane in the constellation. This RAAN shift could be used to lower the overall revisit time.

However; a RAAN shift would not have the effect of increasing the effectivity of a constellation, by increasing the average coverage time while also lowering the standard deviation.

Other augmentations schemes of multiple single launch planes can use the implications presented to create custom multi-plane constellations. By using the high overall coverage of lower inclinations, the high coverage consistency of high inclinations, and RAAN shift, any desired constellation can be created. For specific regions, the specific two plane constellation provides a significant level of even coverage and could be used by future CubeSat constellation developers.

\subsubsection{Augmented Orbit Altitudes}

In order to provide analysis that more closely resembles the likely launch orbits available to a CubeSat constellation the previous analysis is performed again with each orbit plane at a different altitude. The VAFB launch is analyzed at an orbit of $750 \times 800 \mathrm{~km}$, as this altitude is more common for a near-sun synchronous orbit from Vandenberg AFB. The CCAFS - 51.6 launch is analyzed at $300 \times 350 \mathrm{~km}$ altitude as this orbit better reflect a more common altitude that Cubes could be placed in on a launch to the ISS. 
The cubes in the VAFB plane will have a larger ground footprint due to the increased altitude while the cubes in the CCAFS $-51.6^{\circ}$ launch will have a decreased footprint. Due to this effect, the characteristics of the single plane VAFB launch will be more dominant while the CCAFS $51.6^{\circ}$ plane will be less dominant. Table 25 below denotes the coverage metrics for Case 1 and 2 using the augmented orbits.

Table 25: Augmented two plane constellation metrics

\begin{tabular}{|l|c|c|c|c|}
\hline \multirow{2}{*}{$\begin{array}{c}\text { Metric Values } \\
(\text { min) }\end{array}$} & \multicolumn{2}{|c|}{ Case 1 } & \multicolumn{2}{c|}{ Case 2 } \\
\cline { 2 - 5 } & Coverage Time & Revisit Time & Coverage Time & Revisit Time \\
\hline Min & 94.811 & 56.388 & 65.934 & 123.159 \\
\hline Max & 161.798 & 166.253 & 104.352 & 232.868 \\
\hline Average & 117.389 & 105.311 & 79.898 & 176.378 \\
\hline Std. Deviation & 17.048 & 22.849 & 7.469 & 16.433 \\
\hline Sum (days) & 198.093 & 177.712 & 2064.983 & 4558.501 \\
\hline \% Coverage & \multicolumn{2}{|c|}{100} & \multicolumn{2}{c|}{100} \\
\hline
\end{tabular}

When compared to the non-augmented orbit coverage metrics the results of this analysis confirm the expected trends. For Case 1, the average coverage time is approximately 30 minutes lower while the average revisit time is nearly 30 minutes longer. However the standard deviation is nearly half in the coverage time metric and approximately 5 minutes better in the revisit time metric. The same trends are realized for Case 2; with a higher average coverage time and lower average revisit time. Unlike in Case 1; the standard deviation is not significantly lower in the augmented orbit constellation than in the non-augmented constellation.

While the results of the augmented constellation are different from that of non-augmented constellation, the differences are relatively small. This is especially true when comparing the difference in metrics between the two multi-plane constellations to the differences between each of the single plane coverage. 


\section{Conclusion and Future Work}

\subsection{Conclusion}

The analysis shows that it is simple to create a constellation a heavily dispersed constellation of CubeSats by controlling only the launch opportunity, separation direction, and separation magnitude.

\subsubsection{Cube Separation Schemes}

The separation parameters allow for control over the development profile of a constellation in a given orbit. By using different separation geometries different degrees of separation can be created. Deploying cubes using an equal Y-vector distribution leads to a near evenly distributed constellation with a Clusterness value of 0.02099 . However; this deployment scheme requires the rocket body to perform eight different attitude change maneuvers, as the angles between each Cube are not constant. Using the equal angle based deployment scheme yields a constellation that is nearly as separated as the Y-vector deployment scheme, with a Clusterness value of .06014 in the same time period. This deployment scheme requires the rocket body to perform the same maneuver 8 times and is would result in less complexity for deployment. Both of these deployment schemes can be used to deploy a series of CubeSats into near full separation in an orbital plane. When dispersed the cubes drift relative to one another and reach their initial maximum full deployment approximately 40 - 50 days.

\subsubsection{Constellation Breakdown}

After reaching maximum deployment the cubes will continue to drift placing the constellation in a state of oscillation; in which the constellation tends to stay relatively separated but can experience periods of very low separation. It was found that the $\Delta \mathrm{V}$ required to stop the drift of a constellation is extremely manageable and could be used to ensure the constellation stays at its initial full deployment state and does not oscillate. While many developers may want to use an 
orbital maneuver or otherwise change the orbital velocities of the cubes to stop their drift, not all applications require this. For missions that require specific coverage near the beginning and less stringent coverage afterwards no maneuver would be required.

\subsubsection{Launch Opportunities}

As discussed in section 4.3 launch planes of high inclination provides very consistent but low amounts of coverage. Lower inclinations have a hard time providing even coverage to nonequatorial regions, but provide a significant amount of overall coverage for regions they cover. Mid-range inclinations provide higher baseline coverage than high inclinations but provide only nominally better consistency than the low inclination planes. Augmenting a single plane launch with another launch allows the final constellation to inherit traits of both single launch planes. Coverage time will stack evenly while revisit time drops based on how separated the orbital planes are; both RAAN and inclination can be used to affect revisit time. The analysis shows that two launches can provide coverage to a medium sized, high inclination, and high population zone such as Western Europe (Case 1) and a large near equatorial zone (Case 2). Coverage of any point in these areas results in a minimum revisit time of less than one and two hours for Case 1 and 2 respectively. These results extend to a region with the same latitudes allowing a prospective CubeSat constellation developer to use the same launches analyzed to provide ground coverage for any region between $39.4^{\circ} \mathrm{N}$ to $54^{\circ} \mathrm{N}$ longitude (for Case 1 ) and $10^{\circ} \mathrm{N}$ to $35^{\circ} \mathrm{S}$ (for Case 2).

\subsubsection{Tool Development}

In order to produce the discussed results a MATLAB - STK interface GUI called SATCAT 2.0 was created. SATCAT 2.0 can be utilized to analyze any CubeSat deployment scheme for use analysis of future single plane CubeSat deployment in order to properly identify individual cubes well before TLE's can be used. SATCAT 2.0 can also be used by a developer to perform custom analysis on a CubeSat constellation regarding specific mission ground coverage needs. 


\subsection{Future Work}

This thesis has made clear that a few things need additional work required in order to successfully deploy a CubeSat constellation. The lack of knowledge regarding parameters that affect each CubeSat's propagation must be addressed. Information on the Cube's exact cross section, coefficient of drag, and attitude control are critical in predicting the future locations of actual cubes being planned for a constellation. Further information is also required on the separation speed of each cube. The current data provided by CubeSat is insufficient as the analysis used to obtain the data does not include the errors in the separation spring and the micro-gravity environment while the P-POD jettisons the Cubes. The lack of knowledge in these areas leads to an uncertainty in each cube's position in the constellation. While this may not pose an issue for most developers, this is an obstacle for a developer seeking to design a constellation based on high accuracy and specific revisit times.

Another major place for future work is to incorporate different types of orbits as launch opportunities. While most launches that carry CubeSats are generally low earth orbit and circular occasionally highly eccentric or higher altitude launches are available. The ways in which these orbits could be used to augment a constellation could serve as a method for increasing the usability of CubeSat constellations. Due to the limited number of launches into these regimes only longer term missions could benefit from their use. Constellations that have multi-altitude cubes will also no longer benefit from equal drag on all cubes in the constellation. Only the cubes in each altitude band will move together, causing each altitude band to drift with respect to one another. This drift will also need examination in further studies.

Additionally all the coverage metrics are based on all the Cubes performing nominally. Future analysis should research mitigation strategies for cube failures both prior and post full constellation development. Depending on when one or more cubes fails the mitigation strategy could be based in changing when an orbital maneuver will be done to an additional launch. 


\section{References}

[1] The CubeSat Program, Cal Poly SLO, "CubeSat Design Specification, Rev. 12," CubeSat, San Luis Obispo.

[2] CubeSat Kit, "3D CAD Design," [Online]. Available: http://www.cubesatkit.com/content/design.html. [Accessed Dec 2012].

[3] The CubeSat Program, Cal Poly SLO, "CubeSat: About Us," [Online]. Available: http://www.cubesat.org/index.php/about-us. [Accessed Dec 2012].

[4] J. Puig-Suari, C. Turner and W. Ahlgren, Development of the Standard CubeSat Deployer and a CubeSat Class PicoSatellite, vol. 1, IEEE, 2001.

[5] Naval Postgraduate School, "NPS CubeSat Launcher (NPSCuL) Program Update," San Luis Obispo, 2009.

[6] T. Wilcox, "NPS CubeSat Launcher," 2012.

[7] National Coordination Office for Space-Based Positioning, Navigation, and Timing., "Global Position System," 15 Nov 2012. [Online]. Available: http://www.gps.gov/. [Accessed Dec 2012].

[8] Iridium, "The Satellite Constellation," [Online]. Available: http://www.iridium.com/About/IridiumGlobalNetwork/SatelliteConstellation.aspx. [Accessed Dec 2012].

[9] D. Vallado, Fundamentals of Astrodynamics and Applications, Hawhorne: Microcosm Press, 2007.

[10] B. Smalarz, "CUBESAT CONSTELLATION ANALYSIS FOR DATA RELAYING," San Luis Obispo, 2011.

[11] Y. Winetraub and R. Dr Tamir, "Using Differential Drag for management of Pico-Satellite Constellations," Israel Aerospace Industries, ISRAEL.

[12] J. Cutler, J. Springmann, S. Spangelo and H. Bahcivan, "Initial Flight Assessment of the Radio Aurora," in Proceedings of the 25th Annual Small Satellite Conference, 2011.

[13] D. A. Vallado and S. Alfano, "Curvilinear Coordinates for Covariance and Relative Motion Operations," in 2011 AAS/AIAA Astrodynamics Specialist Conference, 2011. 
[14] CubeSat, "Atlas V OUTSAT Launch 2012," [Online]. Available:

http://www.cubesat.org/index.php/missions/upcoming-launches/122-l36-launch-alert. [Accessed February 2013].

[15] A. E. Petropoulos, "Refinements to the Q-law for low-thrust orbit transfers," in 15th AAS/AIAA Space Flight Mechanics Conference, Copper Mountain, 2005.

[16] D. T. Schmuland, M. R. K and C. G. Sota, "Hydrazine Propulsion Module for CubeSats," in 25th Annual AIAA/USU Conference on Small Satellites, 2011.

[17] J. R. Wetz, Orbit \& Constellation Design \& Management, Hawthorne: Microcosm Press, 2009. 


\section{Appendix A - How to Use SATCAT 2.0}

SATCAT 2.0 is a straightforward MATLAB based GUI. It has designed to be modular and functionality can be changed, added, or removed with limited impacts on other modules. Certain modules require that SATCAT and/or STK be in a specific state, which a different module is designed for.

\section{Initializing SATCAT 2.0}

All the commands to initialize SATCAT can be found in the Connection and Scenario modules. The following steps are required to create a New Scenario in STK and begin using SATCAT 2.0. Bold words denote a command that must be selected in SATCAT 2.0.

\section{Open STK}

\section{Connect to STK}

\section{New Scenario}

Note: A "pop-up" window will now open and present three options to the user: Scenario Name, Start Time, End Time. For use in SATCAT 2.0 the usual interval length between start and end time is 1 day. This limits certain propagation times.

4. Input a Scenario Name, Start and End Times, using appropriate format. Select $\mathbf{O K}$

5. A scenario will now be created in STK with SATCAT 2.0's custom features. This includes 3 constellations for Cubes to be placed into later. This also includes two chains as a legacy item from $v 1.0$

Users who already have pre-saved or pre-created STK scenarios should follow steps 1 and 2 above, followed by manually opening the scenario in STK.

\section{Inputting P-POD / Rocket Body}

Once a scenario has been created use the P-POD (Rocket Body) module to add a P-POD. 
1. Input the P-POD's orbit in the Orbit module

2. Verify or change the P-POD's propagation parameters in the Parameters module. The defaults are preset but can be changed.

Note: Be sure to use the rocket bodies parameters, as it dictates the orbit, not the P-POD.

\section{Add P-POD Orbit and Parameters}

4. A satellite named "PPOD" will be added to STK

\section{Inputting Cube Deployments and Propagating}

Adding Cubes to SATCAT 2.0 works in two stages. Stage 1 allows the user to input Cubes into SATCAT before routing them to STK by inputting Cubes into the "Cube Matrix". Stage 2 routes all the cubes to STK sequentially. This allows the user to input all the cubes before issuing a command to STK.

Inputting Cubes

1. Input a dT and a dV, and verify the cube propagation parameters.

2. Decide on any checkboxes to use.

a. Hold Parameters - This cube stops SATCAT from clearing the dT and dV when moving on to the next cube.

b. Body Frame - All deployments will occur in the body frame Note: THIS IS NOT AN INDIVIIDUAL CUBE OPTION. WILL APPLY TO ALL CUBES

c. Unit Vector - Checking this box tells SATCAT that the dV inputted is a unit vector only. The magnitude is found using the inputted mass and the separation data provided by CubeSat at Cal Poly.

\section{Add / Edit Cube}

4. Repeat these steps until all desired cubes are added 
Additional options exist for the user. Selecting Delete Cube will delete the cube currently inputted in the Cube \# input box. For example, to delete Cube 5: input 5 into the Cube \# box and select Delete Cube

A batch upload feature is also built into SATCAT 2.0. Using this feature requires the user to create a Microsoft Excel spreadsheet with the deployment data on the first sheet. The columns must be in the same order that the Cube Matrix button in the Commands module outputs. The file selected must also be in the same folder as the SATCAT 2.0 current folder.

All of the above commands are used to edit SATCAT 2.0's Cube Matrix. This matrix is used to input the cubes into STK. Once the Cube Matrix is finalized in order to input the Cubes into STK, input the

\section{Request Metrics}

Requesting metrics is done through the Metrics module. Some metric commands require the use of the Commands module.

\section{Coverage Metrics}

In order to obtain coverage metrics the following commands must be issued:

1. Load: Case 1 and Case 2

2. Assign Assets (This can only be done AFTER the cubes are propagated in STK)

3. Determine an interval the coverage is being requested for. Input that as a bi-lateral number in the Center and +/- input boxes and select Change Interval.

4. After the interval has been changed select Run Coverage. (this can take a long time, be patient)

5. Reviewing the coverage metrics once the coverage analysis is complete is done in STK. 


\section{Consistency Metrics}

In order to find the constellation consistency the following commands must be issued:

1. Input the \# of cubes being analyzed.

Note: Generally if 8 cubes were propagated then the input would be 8 , however, this feature allows for additional cubes to be added in the STK propagator and not used for consistency calculation. The cubes are selected sequentially. (i.e. 5 cubes means cubes 1 through 5)

2. Input the Start and Stop time spans in days.

\section{Run Consistency}

4. Run the file "PlotConsistency.m" in the SATCAT folder to plot the consistency values. Note: if this file is not present SATCAT 2.0 automatically places the necessary values for plotting in the MATLAB workspace. User can plot the values from there. 


\section{Appendix B - NROL-36 Error}

The figures below depict the error between the JSpOC provided TLEs and the STK analysis with no corrected assumptions.
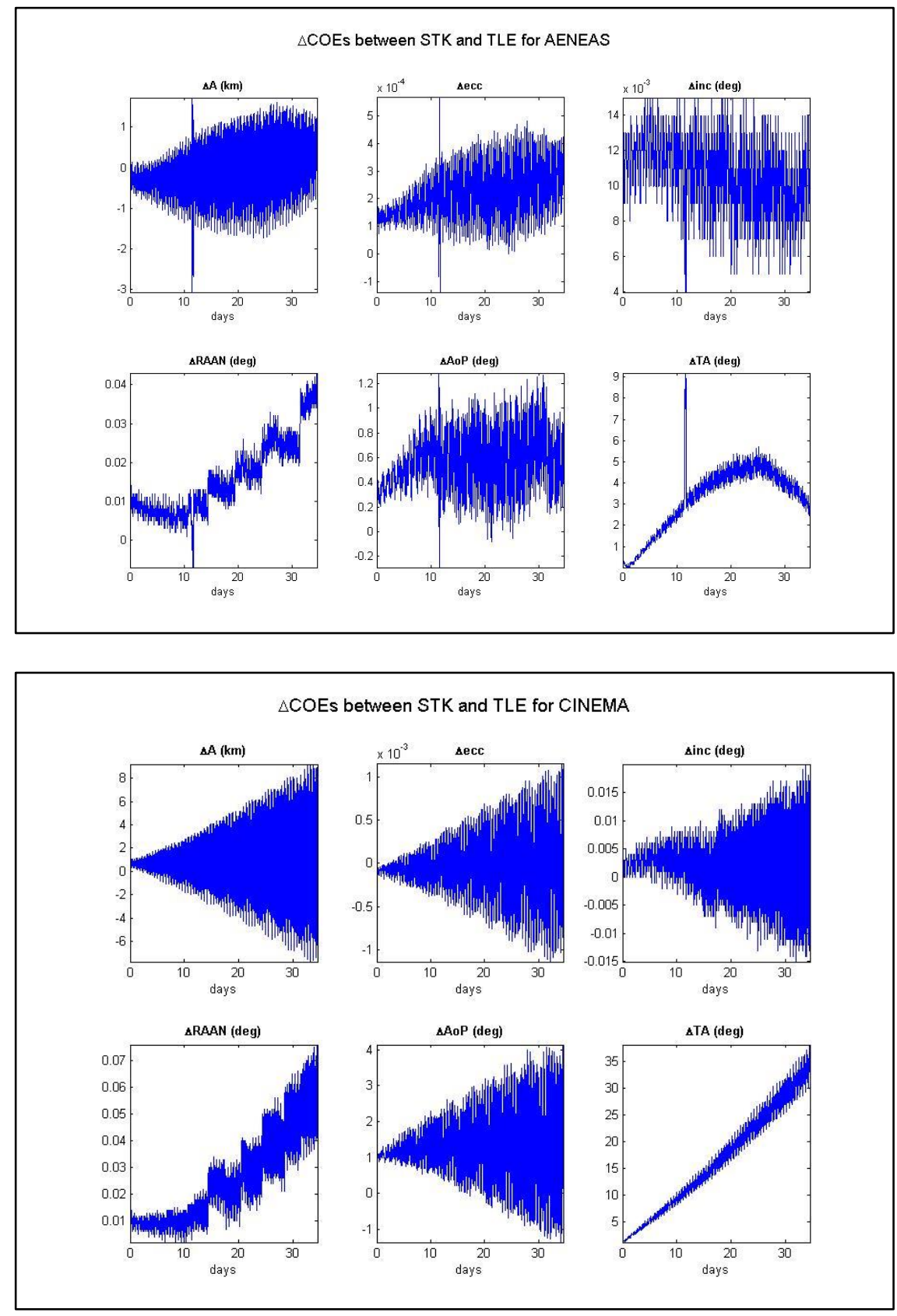

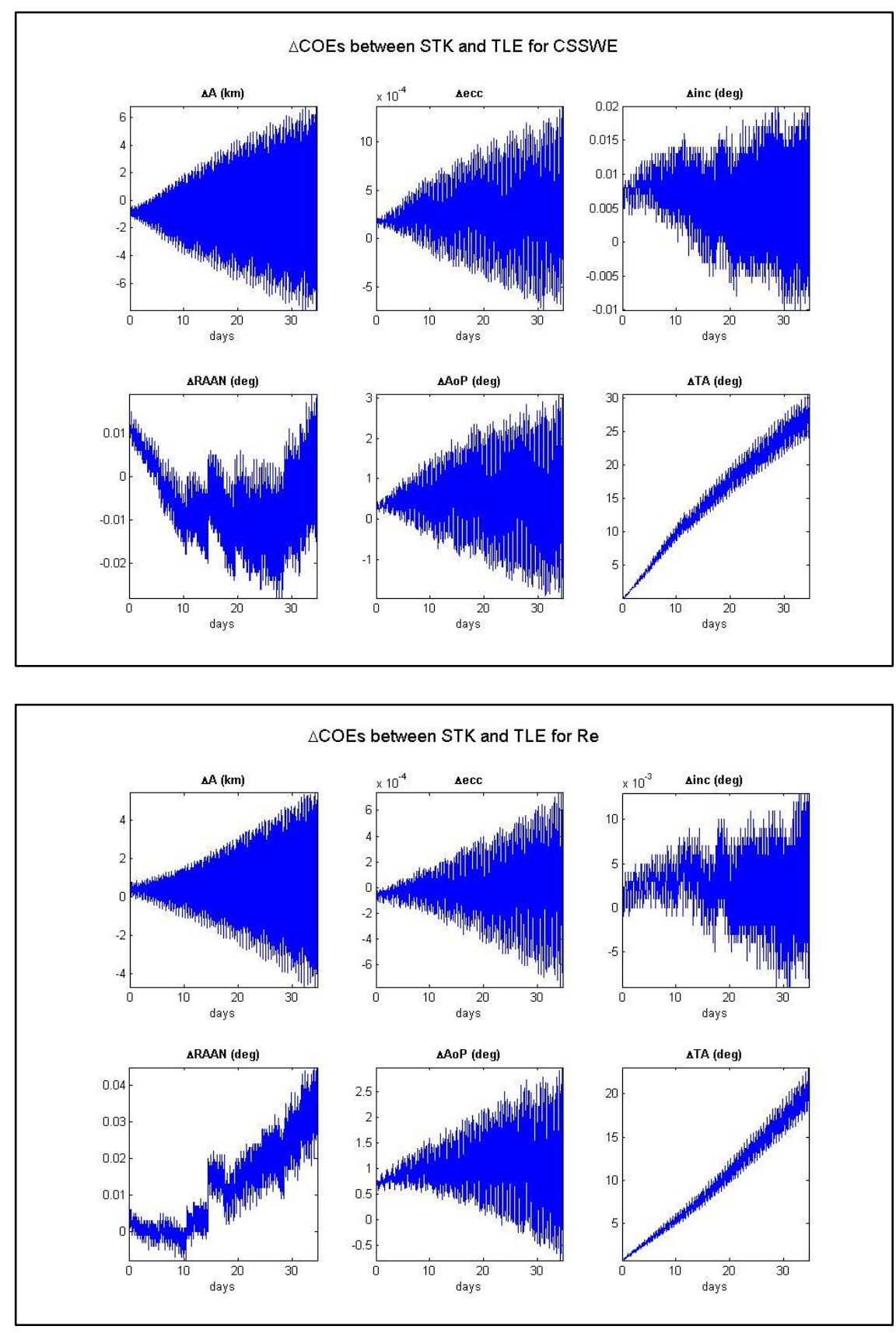

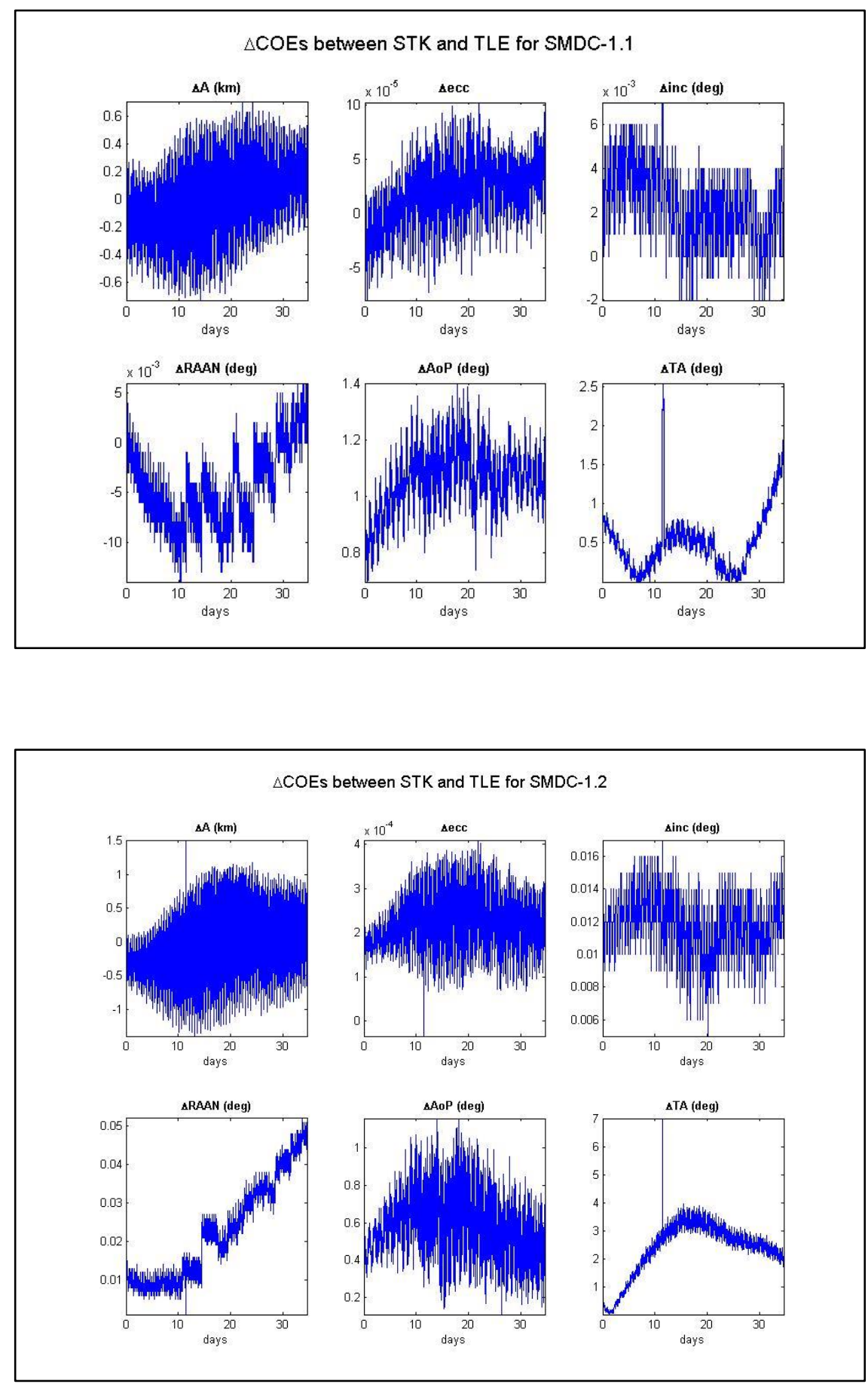

The figures below denote the error between the JSPoC provided TLEs and the STK analysis with changed assumptions. 

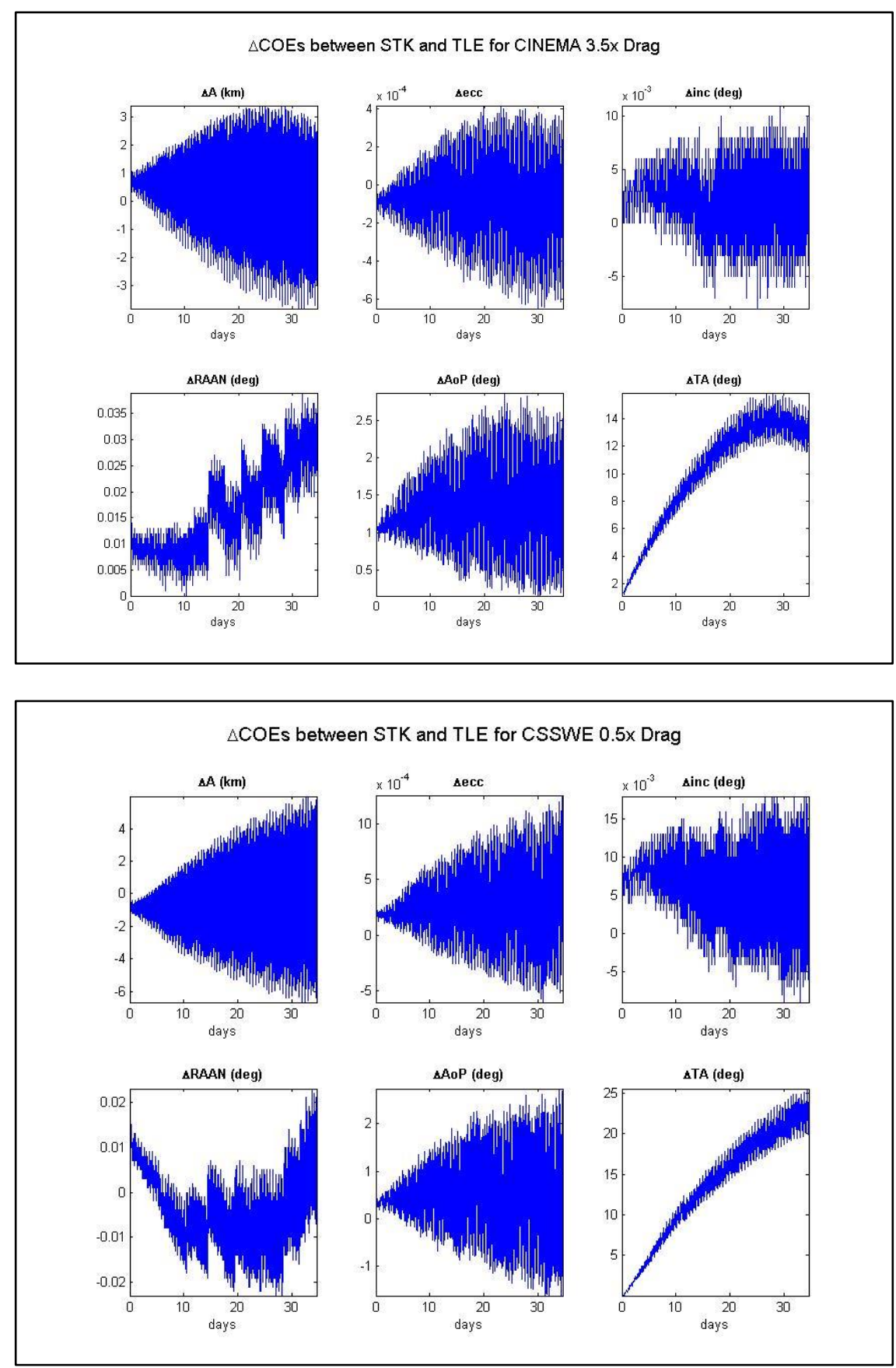


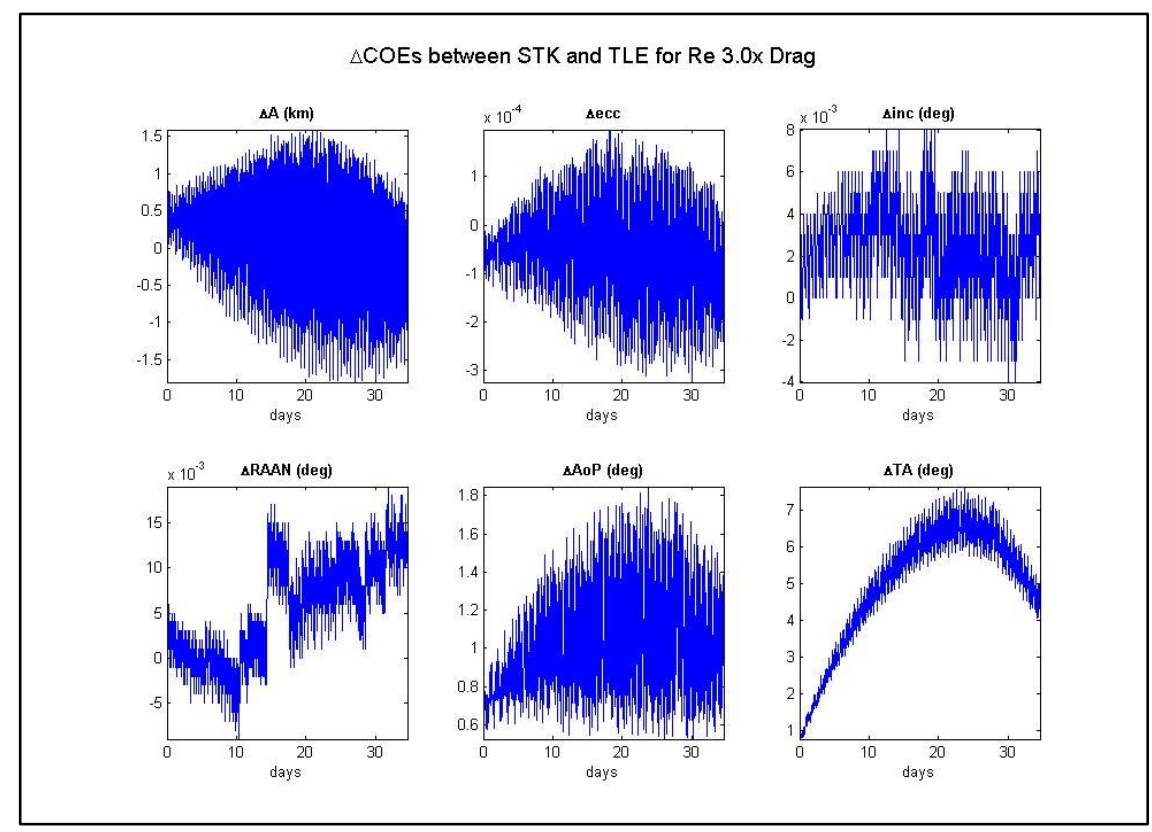

